JOÃO CARLOS MEDAU

ANÁLISE DE CAPACIDADE DO LADO AÉREO DE AEROPORTOS BASEADA EM SIMULAÇÃO COMPUTACIONAL: APLICAÇÃO AO AEROPORTO DE SÃO PAULO - CONGONHAS 
JOÃO CARLOS MEDAU

\section{ANÁLISE DE CAPACIDADE DO LADO AÉREO DE AEROPORTOS BASEADA EM SIMULAÇÃO COMPUTACIONAL: APLICAÇÃO AO AEROPORTO DE SÃO PAULO - CONGONHAS}

Dissertação apresentada à Escola Politécnica da Universidade de São Paulo para obtenção do título de Mestre em Engenharia 
JOÃO CARLOS MEDAU

\section{ANÁLISE DE CAPACIDADE DO LADO AÉREO DE AEROPORTOS BASEADA EM SIMULAÇÃO COMPUTACIONAL: APLICAÇÃO AO AEROPORTO DE SÃO PAULO - CONGONHAS}

Dissertação apresentada à Escola Politécnica da Universidade de São Paulo para obtenção do título de Mestre em Engenharia

Área de Concentração:

Engenharia de Transportes

Orientador:

Prof. Dr. Nicolau D. Fares Gualda 
Este exemplar foi revisado e alterado em relação à versão original, sob responsabilidade única do autor e com a a anuência de seu orientador.

São Paulo, 01 de julho de 2011.

Assinatura do autor

Assinatura do orientador

FICHA CATALOGRÁFICA

\section{Medau, João Carlos}

Análise de capacidade do lado aéreo de aeroportos baseada em simulação computacional: aplicação ao aeroporto de São Paulo - Congonhas / J.C. Medau. - ed.rev. -- São Paulo, 2011. 120p.

Dissertação (Mestrado) - Escola Politécnica da Universidade de São Paulo. Departamento de Engenharia de Transportes.

1. Operações de transportes (Simulação computacional) 2. Aeroportos 3. Capacidade de tráfego 4. Serviços (Nível) I. Universidade de São Paulo. Escola Politécnica. Departamento de Engenharia de Transportes II. t. 


\section{DEDICATÓRIA}

À minha família 


\section{AGRADECIMENTOS}

Ao Professor Nicolau Gualda pela confiança, pelos ensinamentos e pela orientação durante toda a execução deste trabalho, e também por mostrar os desafios empolgantes da Engenharia de Transportes.

Aos Professores Cláudio Barbieri da Cunha e Orlando Strambi pelos ensinamentos.

À Professora Cristina Belderrain pelo constante incentivo desde a graduação para que eu realizasse o Mestrado.

Ao Rodrigo Moser e Gregory Bradford pela ajuda e suporte com o Visual SIMMOD.

Aos amigos, funcionários e colegas do Departamento de Engenharia de Transportes da POLI pela ajuda e convivência durante todo o curso. 


\section{RESUMO}

O tráfego aéreo brasileiro aumentou sensivelmente nos últimos anos e tem previsões de aumento acima da média mundial para as próximas duas décadas, chegando a $7,3 \%$ ao ano. Por outro lado, importantes aeroportos brasileiros já apresentam sinais de congestionamento e políticas de restrição são necessárias para manter os níveis de serviço oferecido às aeronaves e passageiros acima de um limite mínimo aceitável. Este trabalho apresenta uma metodologia baseada em modelos de simulação para analisar a capacidade do lado aéreo de aeroportos. Seu propósito é determinar o fluxo máximo de tráfego, mantendo o nível de serviço acima de um valor pré-definido, levando a atrasos aceitáveis para aeronaves aguardando na fila para decolagem. Para desenvolver o estudo, foi utilizado o software Visual SIMMOD e a metodologia apresentada foi aplicada ao Aeroporto de São Paulo - Congonhas. Os resultados das simulações mostram que o aeroporto está saturado, com um volume de tráfego que leva a um nível de serviço inadequado. Para atingir um nível de serviço adequado, uma redução de tráfego é necessária. Outros cenários de simulação mostram que o nível de serviço pode ser melhorado com mudanças em procedimentos de controle de tráfego aéreo,

envolvendo separações nas decolagens e aproximações, e também com a substituição de aeronaves turbo hélices por jatos.

Palavras Chave: Simulação. Aeroporto. Nível de Serviço. Visual SIMMOD. 


\begin{abstract}
The Brazilian air traffic has increased significantly in recent years and is expected to increase above the world average for the next two decades, reaching $7,3 \%$ per year. Furthermore, major Brazilian airports are already showing signs of congestion and restriction policies are necessary to keep the level of service (LOS) offered to passengers and aircraft above a minimum acceptable. This work presents a methodology based on simulation models to analyze the capacity of the airside of airports. Its purpose is to determine the maximum flow of traffic while keeping LOS above a minimum level, leading to acceptable delays for aircraft waiting in line for takeoff. To develop the study, Visual SIMMOD software was used and the proposed methodology was applied to Sao Paulo - Congonhas Airport. The simulation results show that the airport is saturated with a volume of air traffic that leads to an inadequate LOS. To achieve an adequate LOS, a reduction in traffic is needed. Other simulation scenarios show that the LOS can be improved with changes in ATC procedures involving separations in takeoffs and approaches, and also the replacement of turbo prop aircraft by jets.
\end{abstract}

Keywords: Simulation. Airport. Level of service. Visual SIMMOD. 


\section{LISTA DE FIGURAS}

Figura 1 - Componentes de um aeroporto

Figura 2 - Atrasos para decolagem em função do volume de tráfego ....................... 20

Figura 3 - Balanceamento de capacidade de componentes de um sistema ............. 21

Figura 4 - Regiões de informação de voo do Brasil................................................ 34

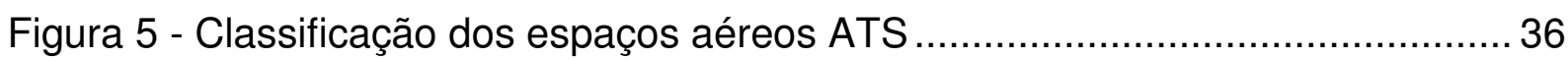

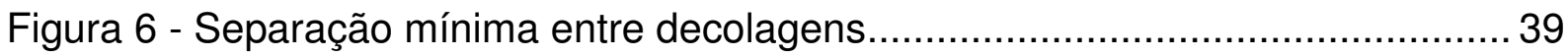

Figura 7 - Separação mínima entre decolagens com cruzamento em altitude........... 39

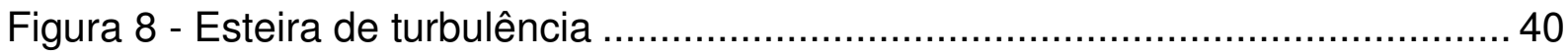

Figura 9 - Separação entre decolagens - 2 minutos ........................................... 42

Figura 10 - Separação entre decolagens de pontos intermediários da pista - 3

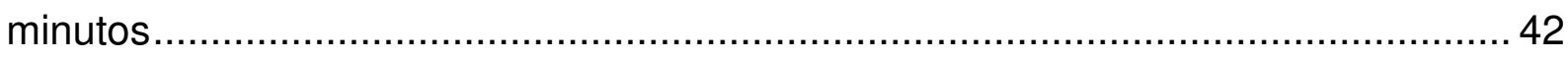

Figura 11 - Localização do Aeroporto de Congonhas ..............................................50

Figura 12 - Comparativo de movimentos realizados e previstos ..............................51

Figura 13 - Planta do Aeroporto de Congonhas ................................................. 52

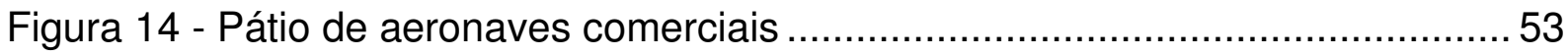

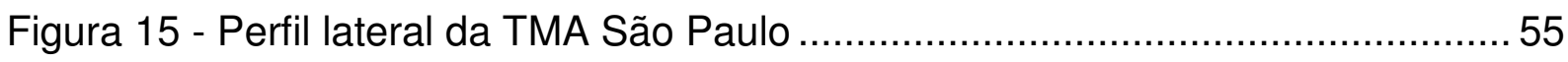

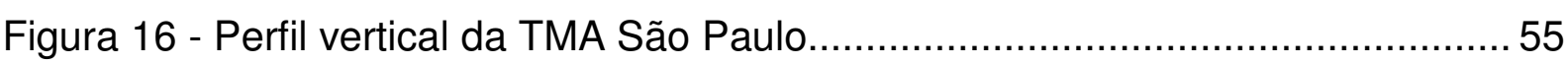

Figura 17 - Representação do Aeroporto de Congonhas no Visual SIMMOD .......... 57

Figura 18 - Rotas de saída (SID) e chegadas (STAR) modeladas no SIMMOD ....... 58

Figura 19 - Movimentos por hora em Congonhas ………................................... 59

Figura 20 - Verificação de rotas de solo com a ferramenta Animator.........................60

Figura 21 - Comparação entre movimentos previstos e simulados.............................61

Figura 22 - Distribuição atual de slots e envelopes de capacidade de Congonhas... 63

Figura 23 - Atraso médio em função do volume de tráfego - separação de 7NM / 90

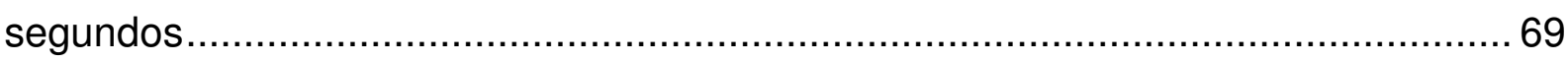

Figura 24 - Decomposição do atraso médio para decolagem - separação 7NM / 90

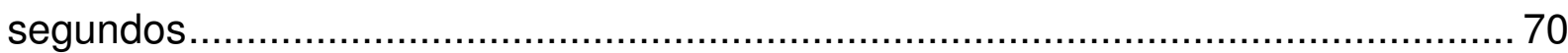

Figura 25 - Taxa de ocupação das posições de estacionamento - cenário 7NM / 90

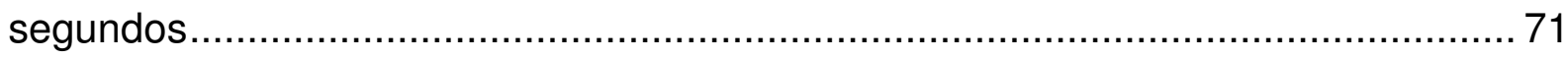

Figura 26 - Comparação entre os cenários simulados ……………...................... 73 
Figura 27 - Comparação entre os cenários com substituição de turbo-hélices por

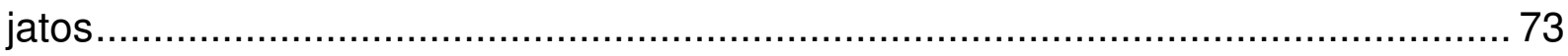

Figura 28 - Comparação entre os cenários com reorganização de slots ..................74 Figura 29 - Diferenças entre movimentos previstos e realizados no cenário de

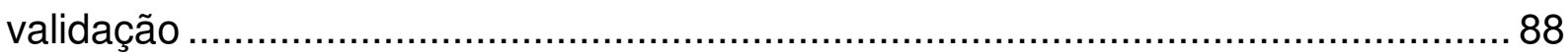

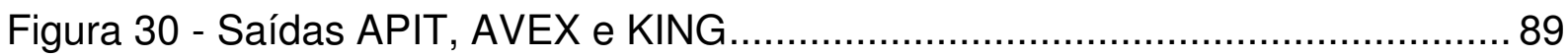

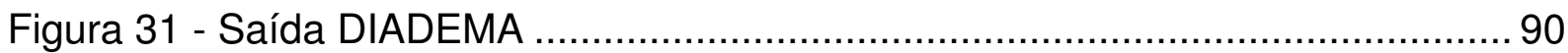

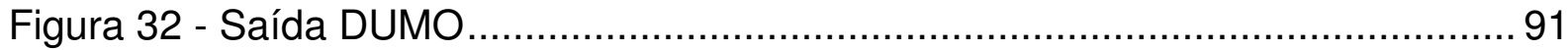

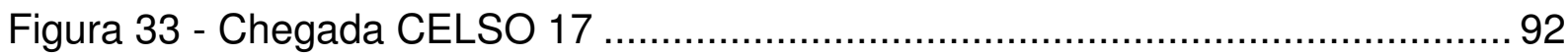

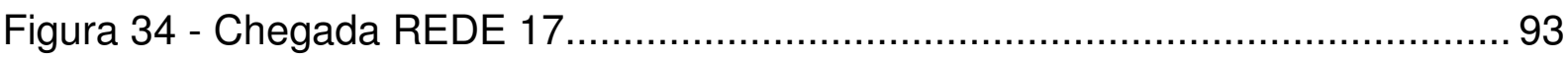

Figura 35 - Turn around time para aeronaves ATR 42-300.................................. 94

Figura 36 - Turn around time para aeronaves Airbus A319 …............................. 95

Figura 37 - Turn around time para aeronaves Airbus A320 ………………….... 96

Figura 38 - Turn around time para aeronaves Boeing 737-100/200/300/400/500 .... 97

Figura 39 - Turn around time para aeronaves Boeing 737-700 ............................ 98

Figura 40 - Turn around time para aeronaves Boeing 737-800 ............................. 99

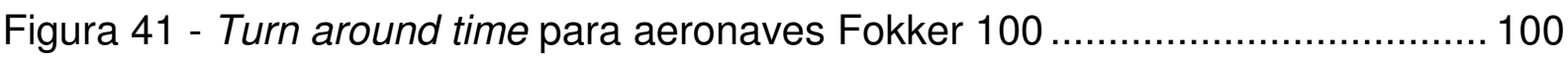




\section{LISTA DE TABELAS}

Tabela 1 - Nível de serviço em função do atraso médio dos voos ........................... 20

Tabela 2 - Mínimos de separação de esteira de turbulência ................................. 41

Tabela 3 - Separação mínima entre aeronaves em aproximação para Congonhas.. 56

Tabela 4 - Alocação de slots para voos comerciais ......................................... 59

Tabela 5 - Quantidade de voos simulados por rota......................................... 61

Tabela 6 - Atraso médio - cenário simulado: 7NM / 90 seg................................. 63

Tabela 7 - Atraso médio - cenário simulado: 7NM / 60 seg ................................... 64

Tabela 8 - Atraso médio - cenário simulado: 5NM / 90 seg................................. 65

Tabela 9 - Atraso médio - cenário simulado: 5NM / 60 seg.................................6 66

Tabela 10 - Atraso médio - cenário simulado: aeronaves a jato.............................. 66

Tabela 11 - Exemplo de decolagens sucessivas usando a mesma SID .................67 67

Tabela 12 - Atraso médio por cenário simulado - reorganização de slots ...............67 67

Tabela 13 - Comparação entre os cenários simulados ........................................ 72

Tabela 14 - Movimentos previstos e realizados no cenário de validação..................87 87 


\section{LISTA DE ABREVIATURAS E SIGLAS}

ANAC

APP

ATM

ATS

ATZ

AWY

CAA

CASA

CGNA

CTR

DECEA

DEP

DME

FAA

FIR

GNSS

GPS
Agência Nacional de Aviação Civil

Approach Control (Controle de Aproximação)

Air Traffic Management (Gerenciamento de Tráfego Aéreo)

Air Traffic Service (Serviço de Tráfego Aéreo)

Air Traffic Zone (Zona de Tráfego de Aeródromo)

Airway (Aerovia)

Civil Aviation Authority (Autoridade de Aviação Civil)

Civil Aviation Safety Authority (Autoridade de Segurança de Aviação Civil)

Centro de Gerenciamento de Navegação Aérea

Control Zone (Zona de Controle)

Departamento de Controle do Espaço Aéreo

Departure (Decolagem)

Distance Measurement Equipment (Equipamento Medidor de Distância)

EUROCONTROL European Organization for the Safety of Air Navigation (Organização Européia para Segurança da Navegação Aérea)

Federal Aviation Administration (Administração Federal de Aviação)

Flight Information Region (Região de Informação de Voo)

Global Navigation Satellite System (Sistema de Navegação Global por Satélite)

Global Positioning System (Sistema de Posicionamento Global) 


\begin{tabular}{|c|c|}
\hline IATA & $\begin{array}{l}\text { International Air Transportation Association (Associação } \\
\text { Internacional de Transporte Aéreo) }\end{array}$ \\
\hline ICA & Instrução do Comando da Aeronáutica \\
\hline ICAO & $\begin{array}{l}\text { International Civil Aviation Organization (Organização de } \\
\text { Aviação Civil Internacional) }\end{array}$ \\
\hline IFR & Instrument Flight Rules (Regras de Voo por Instrumentos) \\
\hline ILS & $\begin{array}{l}\text { Instrument Landing System (Sistema de Pouso por } \\
\text { Instrumentos) }\end{array}$ \\
\hline LDG & Landing (Pouso) \\
\hline MTC & Metropolitan Transportation Comission \\
\hline NATS & $\begin{array}{l}\text { National Air Traffic Services (Serviços de Tráfego Aéreo } \\
\text { Nacionais) }\end{array}$ \\
\hline PBN & $\begin{array}{l}\text { Performance Based Navigation (Navegação Baseada em } \\
\text { Performance) }\end{array}$ \\
\hline PRM & Precision Runway Monitor (Monitoramento Preciso de Pista) \\
\hline REA & Rotas Especiais de Aeronaves em Voo Visual \\
\hline $\mathrm{REH}$ & Rotas Especiais de Helicópteros \\
\hline SID & $\begin{array}{l}\text { Standard Instrument Departure (Subida Padrão por } \\
\text { Instrumentos) }\end{array}$ \\
\hline SOIA & $\begin{array}{l}\text { Simultaneous Offset Instrument Approach (Aproximações por } \\
\text { Instrumentos Simultâneas e Defasadas) }\end{array}$ \\
\hline STAR & Standard Terminal Arrival Route \\
\hline INFRAERO & Empresa Brasileira de Infra-estrutura Aeroportuária \\
\hline TMA & Terminal Control Area (Área de Controle Terminal) \\
\hline
\end{tabular}


UIR

Upper Flight Information Region (Região Superior de Informação de Voo)

VAL Visual Approach Chart (Carta de Aproximação Visual)

VFR Visual Flight Rules (Regras de Voo Visual)

VOR VHF Omnidirectional Range 


\section{SUMÁRIO}

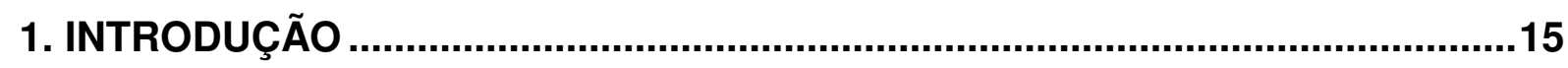

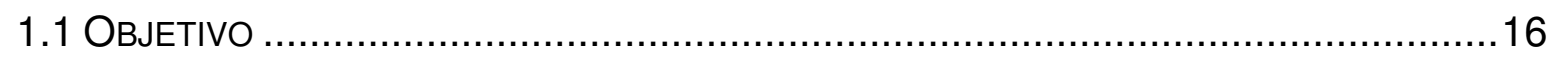

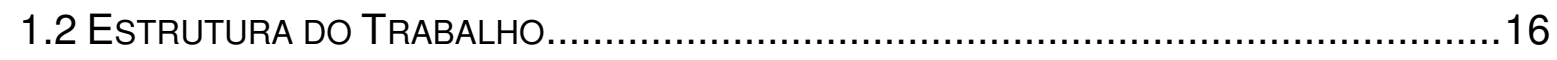

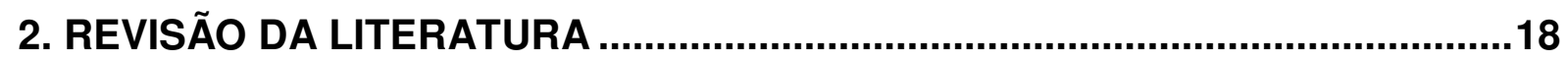

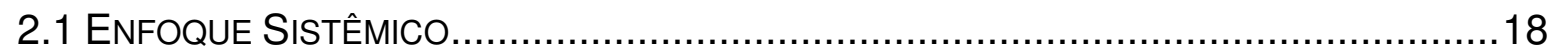

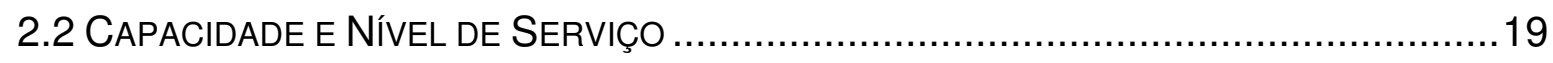

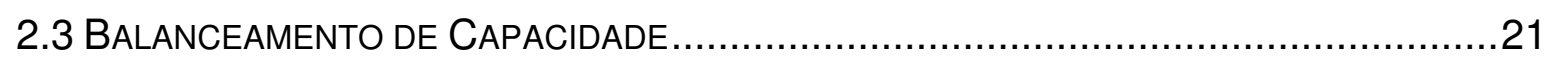

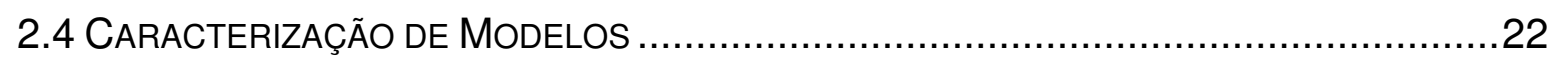

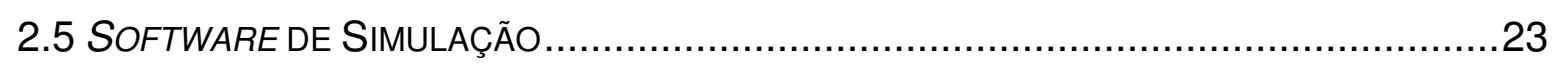

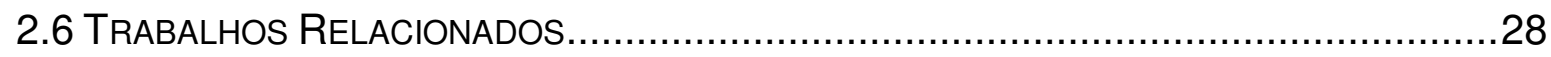

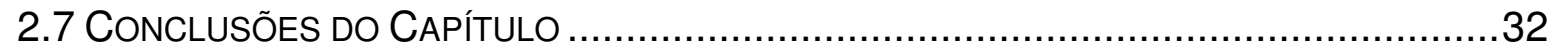

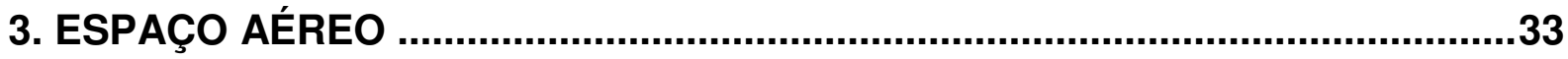

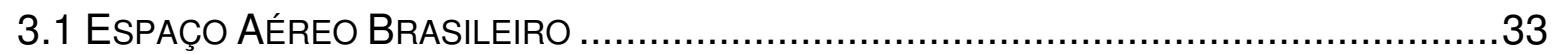

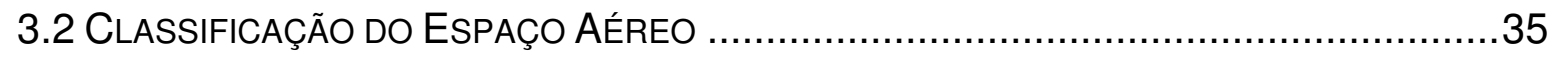

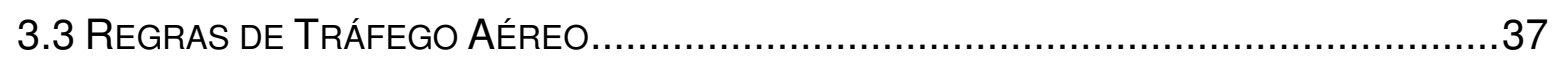

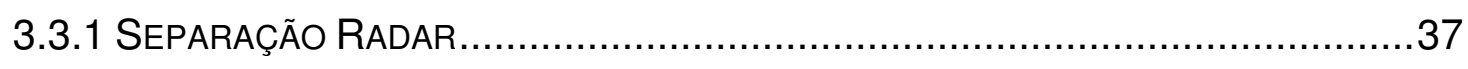

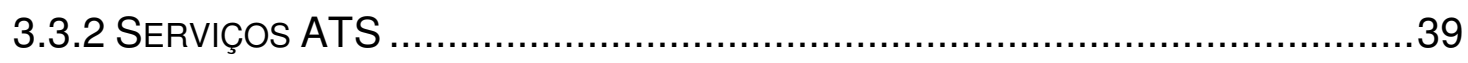

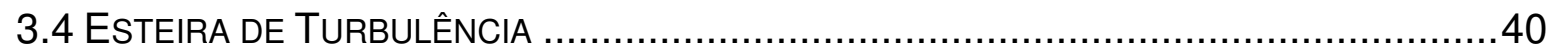

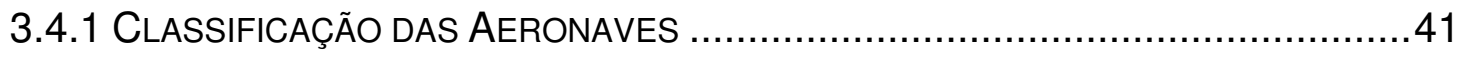

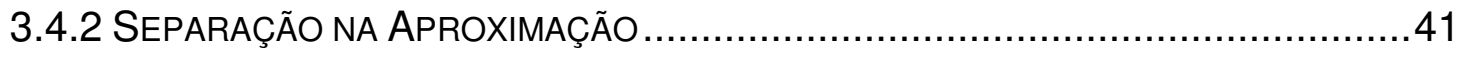

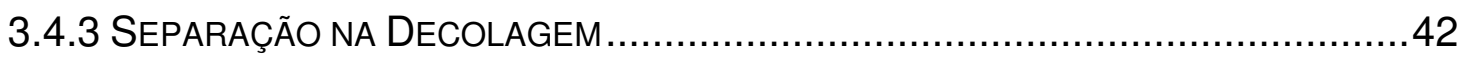

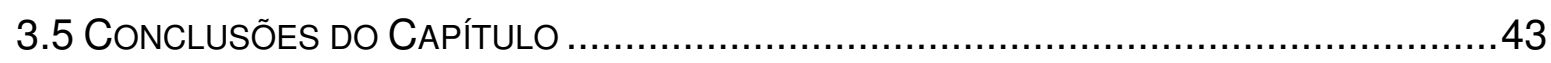

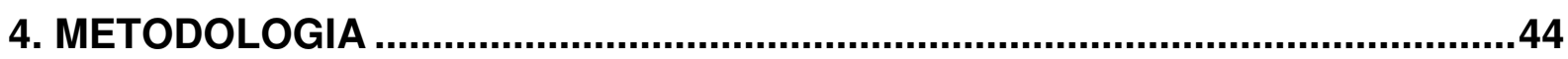

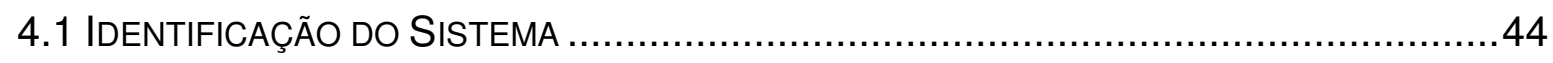

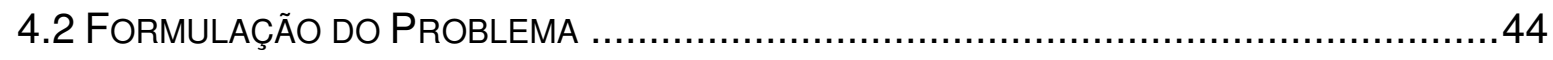

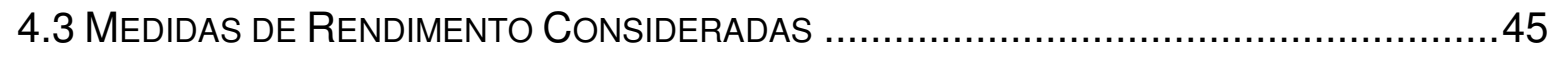

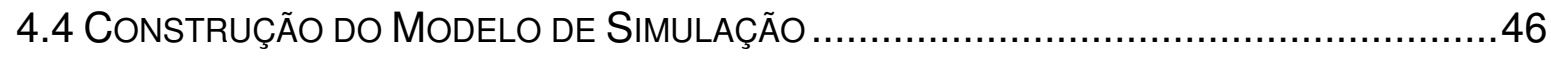

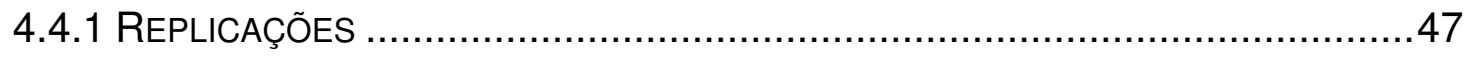

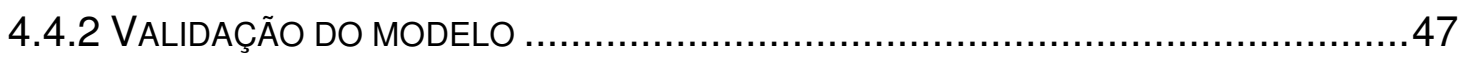

4.5 GeraÇão de Alternativas Para SoluÇão do Problema .................................47 


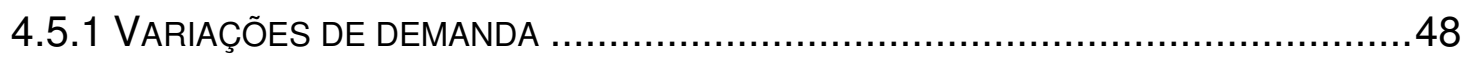

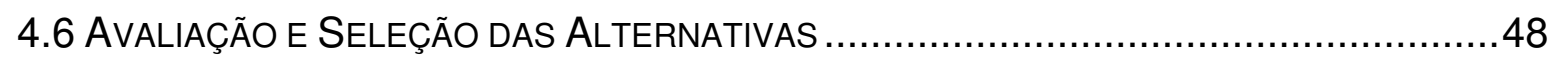

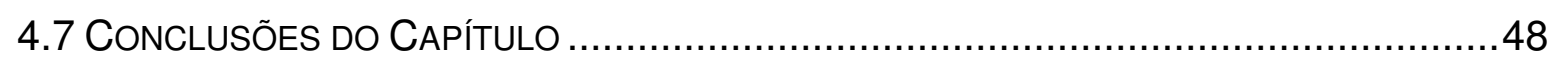

5. APLICAÇÃO AO AEROPORTO DE CONGONHAS ….......................................

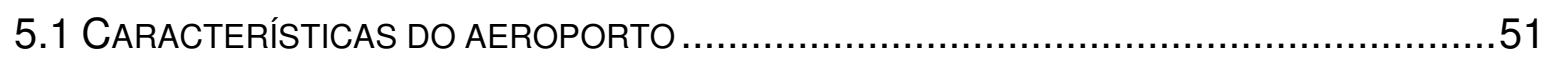

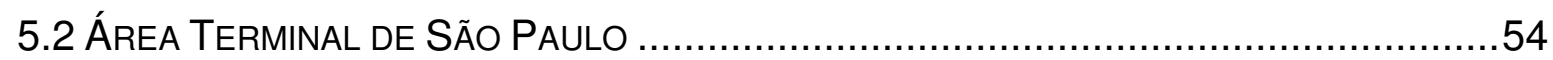

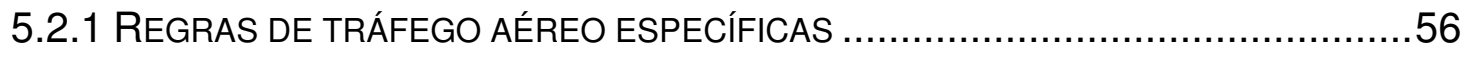

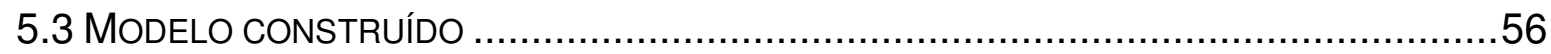

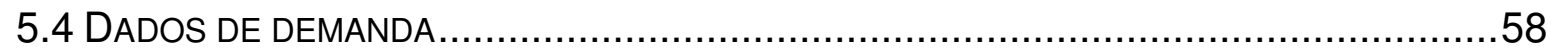

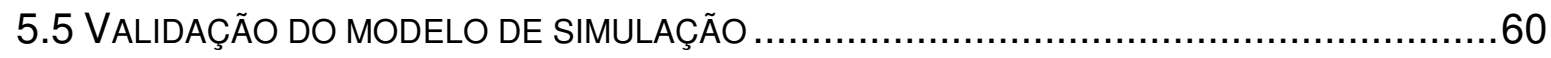

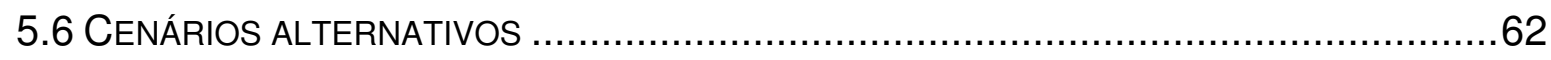

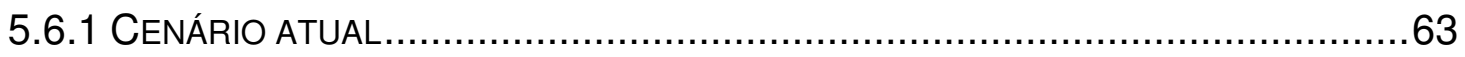

5.6.2 SEPARAÇÃO DE 60 SEGUNDOS ENTRE DECOLAGENS..................................64

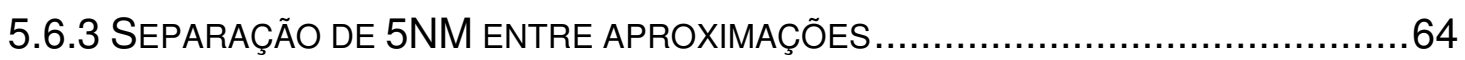

5.6.4 COMBINAÇÃO DOS CENÁRIOS DE 60 SEGUNDOS E 5NM ............................65

5.6.5 SUBSTITUIÇÃO DE AERONAVES TURBO-HÉLICES POR JATOS ..........................65

5.6.6 REORGANIZAÇÃO DA TABELA DE ALOCAÇÃO DE SLOTS ...............................66

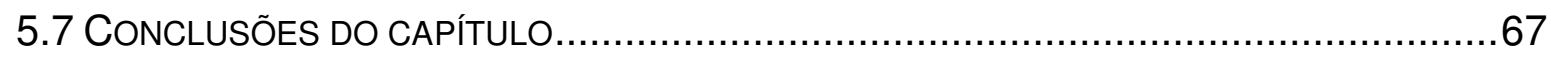

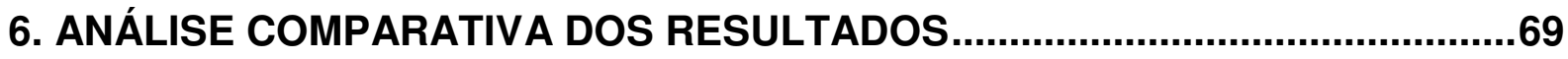

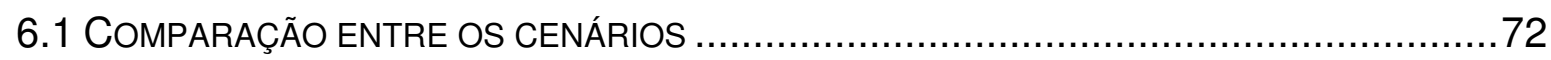

6.2 SUBSTITUIÇÃO DE AERONAVES TURBO-HÉLICES POR JATOS ................................72

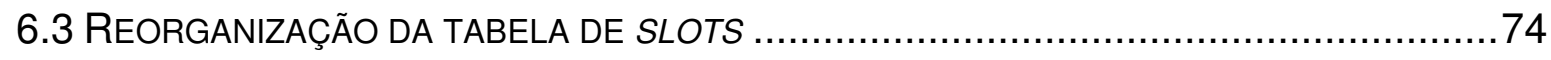

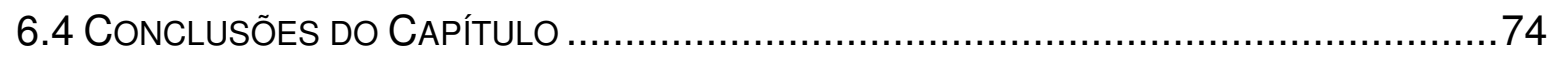

7. CONCLUSÕES, RECOMENDAÇÕES E TRABALHOS FUTUROS ....................76

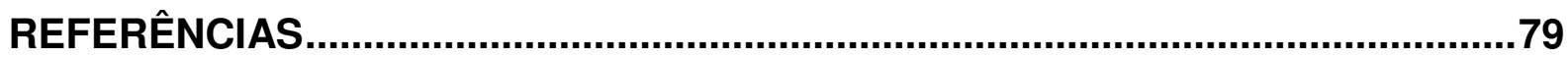

APÊNDICE A - FUNCIONAMENTO DO SOFTWARE SIMMOD ............................84

APÊNDICE B - VALIDAÇÃO DO MODELO DE SIMULAÇÃO …...........................87

ANEXO A - SAÍDAS (SID) E CHEGADAS (STAR) DE CONGONHAS....................89

ANEXO B - DIAGRAMAS DE OCUPAÇÃO DO ESTACIONAMENTO ....................94

ANEXO C - TABELAS DE VOOS SIMULADOS ….............................................101 


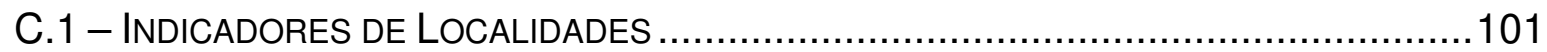

C.2 - AloCAÇÃO DE SLOTS PARA VOOS COMERCIAIS EM CONGONHAS .......................102

C.3 - VOOS EXCLUÍDOS PARA REDUÇÃO DE 5\% NO VOLUME DE TRÁFEGO...................114

C. 4 - VOOS EXCLUÍDOS PARA REDUÇÃO DE 10\% NO VOLUME DE TRÁFEGO ...................115

C.5 - VOOS EXCLUÍDOS PARA REDUÇÃO DE 15\% NO VOLUME DE TRÁFEGO .................117

C.6 - VOOS COM HORÁRIOS REORGANIZADOS ...................................................119 


\section{INTRODUÇÃO}

Estudos realizados pela indústria aeronáutica apontam que a crise econômica de 2009 foi superada, tendo causado uma redução de $2 \%$ no tráfego de passageiros nesse ano. Com a retomada do crescimento, a projeção de expansão da economia global é de 3,2\% ao ano até 2029 e, nesse contexto, a América Latina tem uma projeção de crescimento acima da média mundial, chegando a 4\% ao ano (BOEING, 2010).

Acompanhando as tendências de crescimento da economia, os maiores fabricantes de aeronaves comerciais (Boeing e Airbus) projetam um aumento de quase 100\% na frota mundial em 20 anos, passando de 19.000 aeronaves em 2009 para mais de 36.000 em 2029, sendo que $77 \%$ desse aumento de frota é de jatos comerciais médios (single aisle aircraft).

No Brasil, o tráfego de passageiros apresenta um crescimento de $7,3 \%$ ao ano, com projeções de aumento no tráfego de aeronaves chegando a mais de 3.400 .000 movimentos de decolagens e pousos em 2025 (COMANDO DA AERONÁUTICA, 2005). Outros estudos, porém, apontam que os aeroportos brasileiros já apresentam sinais de congestionamento, não sendo capazes de absorver a demanda prevista para o futuro. Esta deficiência poderá levar a sérias restrições no tráfego aéreo e até mesmo a reduções no crescimento econômico, caso investimentos não sejam realizados para construção e ampliação de aeroportos (EMBRAER, 2010).

As previsões de aumento de demanda e congestionamentos podem levar a uma degradação do nível de serviço oferecido a aeronaves e passageiros. Nesse sentido, ferramentas de avaliação de capacidade e nível de serviço têm um papel importante, ajudando a determinar gargalos e apontando possíveis soluções para adequação da capacidade da infra-estrutura aeroportuária instalada. 


\subsection{Objetivo}

O objetivo deste trabalho é apresentar uma metodologia de avaliação de capacidade associada a nível de serviço do lado aéreo de aeroportos, incluindo pátios de estacionamento, pistas de táxi, pistas de pouso e espaço aéreo adjacente e, também, aplicar a metodologia descrita ao Aeroporto de São Paulo - Congonhas.

As análises de capacidade serão realizadas no cenário de operação atual, considerando a demanda presente e as regras de tráfego aéreo aplicadas, bem como em cenários com aumento e diminuição de demanda, variações nas regras de tráfego aéreo, especialmente em relação aos mínimos de separação aplicados às aeronaves e, também, impactos associados a trocas de modelos de aeronaves.

\subsection{Estrutura do Trabalho}

O capítulo 2 apresenta os conceitos necessários para o desenvolvimento do estudo, fruto de pesquisa na literatura existente, incluindo abordagem sistêmica, capacidade associada a nível de serviço, modelos de simulação, software de simulação, bem como uma revisão de diversos outros trabalhos que empregam simulação aeroportuária.

O capítulo 3 apresenta uma revisão a respeito de classificação do espaço aéreo e das regras de tráfego aéreo aplicáveis, com ênfase àquelas que afetam as aeronaves quando no entorno de aeroportos, durante as fases de saída ou aproximação.

O capítulo 4 apresenta a descrição da metodologia proposta com a aplicação do método científico e do enfoque sistêmico, incluindo a formulação do problema, a definição das medidas de desempenho utilizadas, a criação dos cenários de simulação, a geração, a análise e a escolha de alternativas.

O capítulo 5 inicia-se com a apresentação das características do Aeroporto de Congonhas, além de trazer a aplicação da metodologia proposta a esse aeroporto, com detalhes da construção e validação de um modelo base. Em seguida, são 
apresentados cenários alternativos, com variações na demanda, modificações na separação entre decolagens, entre pousos, a combinação das mudanças de separação com os diferentes volumes de aeronaves e combinações de mudanças de separação, variações na demanda e substituição de aeronaves turbo-hélices por jatos.

O capítulo 6 apresenta a análise dos resultados obtidos nas simulações.

O capítulo 7 apresenta as conclusões e recomendações para trabalhos futuros. 


\section{REVISÃO DA LITERATURA}

\subsection{Enfoque Sistêmico}

Sistema pode ser definido como uma coleção de componentes, conectados por algum tipo de interação ou relacionamento, sendo capaz de responder a estímulos ou demandas e de realizar algum propósito ou função (MEREDITH et al. apud GUALDA, 1995). Para realizar estudos acerca de um aeroporto é necessário, inicialmente, identificar seus subsistemas e componentes.

Um aeroporto pode ser considerado um sistema e dividido em subsistemas, onde destacam-se o lado terrestre e o lado aéreo. O lado aéreo, objeto de estudo deste trabalho, é composto pelas pistas de pouso, pistas de táxi, pátios de estacionamento, baías de espera e o espaço aéreo adjacente ao aeroporto. A figura 1 mostra a divisão de um aeroporto e seus principais componentes.

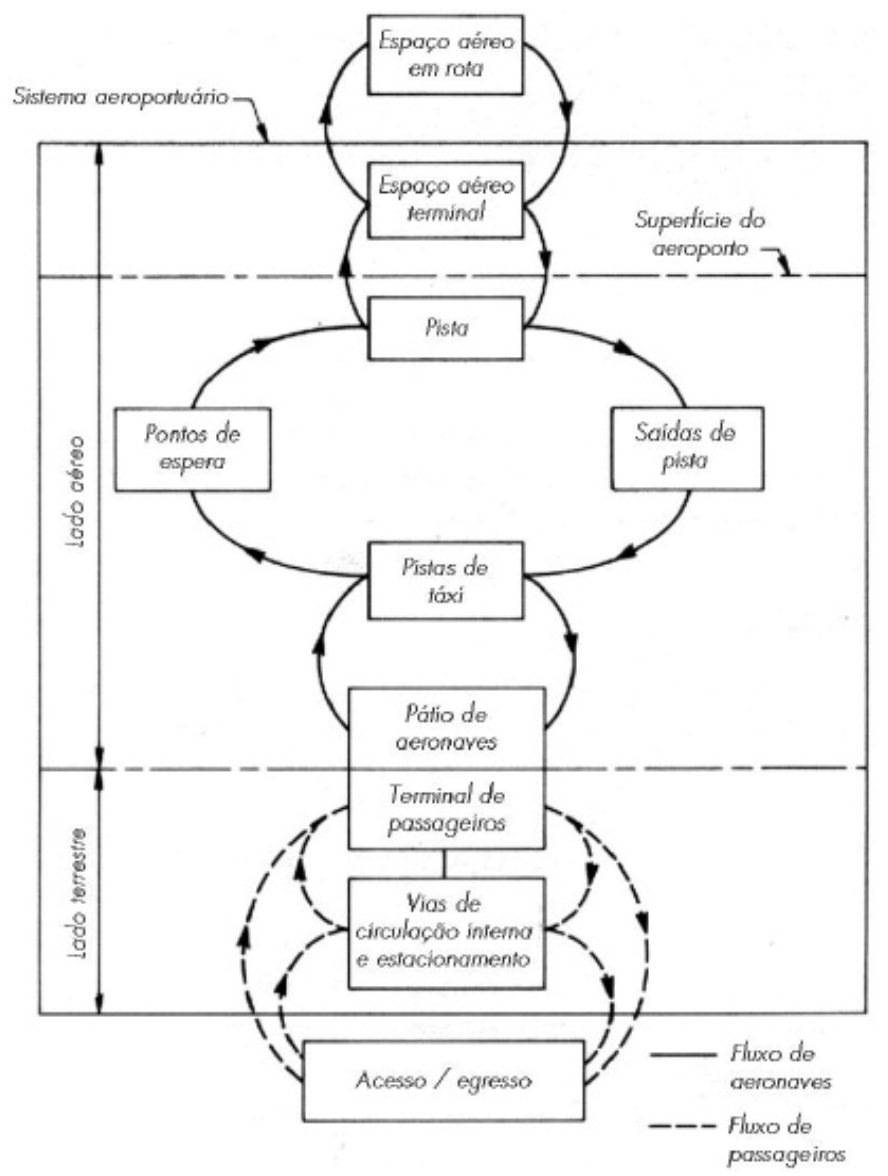

Figura 1 - Componentes de um aeroporto

Fonte: (MOSER, 2007) 


\subsection{Capacidade e Nível de Serviço}

Para a realização de estudos de capacidade de um sistema é necessário definir quais os níveis de serviço que deverão ser oferecidos pelo sistema aos seus usuários. Existem na literatura diversas definições de nível de serviço. No estudo de terminais de passageiros, Müller (1987) define nível de serviço como a percepção dos passageiros em relação à experiência a que foram submetidos no terminal. Kazda e Caves (2008) e Alves (1981) apresentam uma escala adotada pela IATA que relaciona o nível de serviço oferecido aos passageiros com a área dos recintos do terminal destinados a cada atividade. De uma forma mais genérica, Gualda (1978 e 1995) considera nível de serviço como a qualidade do serviço oferecido pelo terminal e que pode ser medido, como exemplo, por comprimentos de filas a serem enfrentadas pelos passageiros, pelas cargas ou pelos veículos que os transportam, por tempos de espera em fila ou aguardando um serviço, por tempos de processamento ou de atendimento, e pelo conforto oferecido aos usuários do terminal.

Para cada componente de um sistema é necessário utilizar uma medida adequada de nível de serviço. Para componentes do terminal de passageiros, pode-se considerar medidas de filas de passageiros ou de percepção de conforto. Já para o lado aéreo, pode-se utilizar o comprimento de filas de aeronaves ou atrasos sofridos pelas aeronaves que pretendem decolar ou aguardam uma posição de estacionamento, após o pouso.

Capacidade de um terminal ou de seus subsistemas pode ser entendida como 0 nível máximo de um tipo de demanda que pode ser imposto ao terminal, em um dado período de tempo, sem violar nenhum dos critérios de níveis de serviço estipulados para o terminal como um todo ou para qualquer dos seus subsistemas ou componentes (GUALDA, 1995).

Para o lado aéreo de um aeroporto, pode-se definir sua capacidade como o número máximo de aeronaves que podem operar no aeroporto em um dado período, sem que $\mathrm{o}$ atraso imposto às aeronaves exceda um valor máximo estipulado. A ICAO recomenda que a capacidade seja definida em termos de quantidade de movimentos 
por hora, podendo, depois, ser estendida a valores diários, mensais, anuais, etc. (ICAO, 2007).

Estudos mostram que os atrasos aumentam exponencialmente quando um sistema opera próximo ao limite de capacidade. A figura 2 mostra a relação teórica entre o aumento de atrasos de aeronaves aguardando para decolagem em função do número de movimentos na pista de pouso.

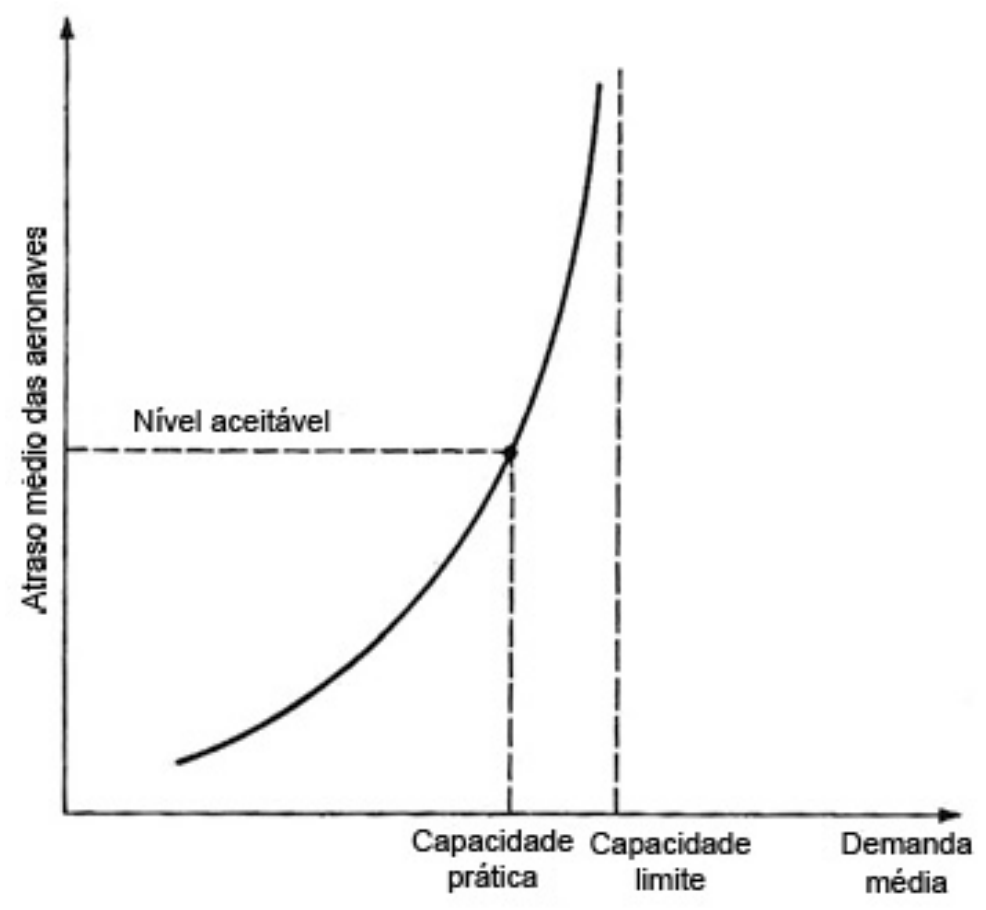

Figura 2 - Atrasos para decolagem em função do volume de tráfego Fonte: Adaptado de HORONJEFF e MCKELVEY (1994)

Em estudos realizados para os aeroportos da área de São Francisco, Estados Unidos, a MTC (2000) adota a escala de nível de serviço apresentada na tabela 1.

Tabela 1 - Nível de serviço em função do atraso médio dos voos Fonte: (MTC, 2000)

\begin{tabular}{cc}
\hline Tempo médio de atraso & Nível de Serviço \\
\hline 0 a 3 minutos & Fluxo livre com pequenos efeitos \\
3 a 5 minutos & adversos no sistema \\
5 a 7 minutos & Aceitável \\
7 a 9 minutos & Congestionado \\
Mais de 9 minutos & Seriamente congestionado \\
\hline
\end{tabular}


Combinando-se as definições anteriores, pode-se afirmar que a capacidade do lado aéreo de um aeroporto é a quantidade máxima de aeronaves que podem operar em uma hora sem que o atraso médio sofrido pelas aeronaves ultrapasse cinco minutos.

\subsection{Balanceamento de Capacidade}

Uma vez que a capacidade de um terminal está limitada pelo componente mais restritivo, é importante que a capacidade dos diversos componentes esteja balanceada. No âmbito do lado aéreo de um aeroporto, deve-se, então, analisar o balanceamento entre as capacidades das pistas de pouso, das pistas de táxi, das posições de estacionamento e do espaço aéreo por onde chegam e saem aeronaves. A Figura 3 ilustra o conceito de capacidade associada a níveis de serviço e o balanceamento de capacidades necessário entre os diversos componentes de um sistema. A figura mostra que os componentes 1 e $n$ estão operando com sobra de capacidade, com consequente folga de nível de serviço. Já o componente 2 está operando no limite de sua capacidade e, nessas condições, qualquer aumento de demanda no subsistema $i$ implicará violação do nível de serviço deste componente. Sendo assim, pode-se afirmar que o terminal está operando no limite de sua capacidade, imposto pelo componente 2 .

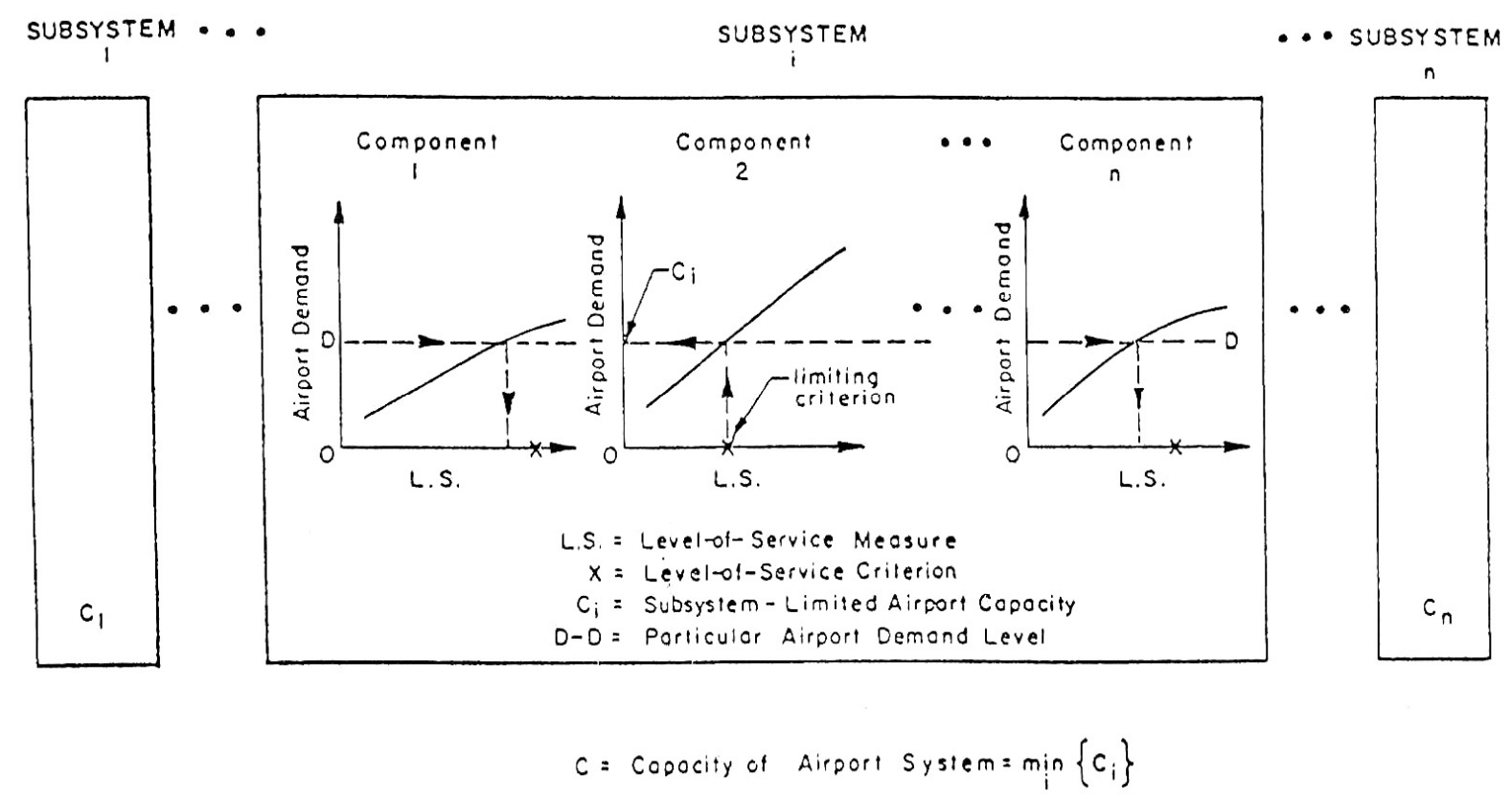

Figura 3 - Balanceamento de capacidade de componentes de um sistema

Fonte: (GUALDA, 1978) 


\subsection{Caracterização de Modelos}

Um modelo pode ser definido como uma representação explícita e simplificada de parte do mundo real - o sistema que se deseja estudar - concentrado em certos elementos considerados importantes para sua análise, por pessoas que desejam usá-lo para entender, modificar, administrar e controlar parte da realidade. (ORTÚZAR e WILLUMSEN, 2001 e PIDD, 1999).

Gualda (1995) divide os modelos da seguinte forma:

- Icônicos: Também conhecidos como modelos físicos, são representações de um sistema construídas em escala, como as maquetes;

- Analógicos: São representações esquemáticas, como desenhos ou plantas baixas;

- Matemáticos: Representações de problemas por meio de fórmulas matemáticas, onde enquadram-se os modelos oriundos da pesquisa operacional.

Santana (2002) e Barragan (2009) dividem os modelos de análise de aeroportos em três categorias:

- Empíricos: Modelos baseados em pesquisas de tráfego, a partir das quais são construídos gráficos e tabelas para a estimativa de capacidade;

- Analíticos: Modelos matemáticos, baseados na Teoria de Filas, que utilizam fórmulas para calcular a capacidade de componentes, como o conjunto de pistas de pousos ou o pátio de estacionamento. Um exemplo é o modelo chamado Airport Capacity Model, desenvolvido pela FAA. Trata-se de um software que calcula a taxa máxima de operações de pousos e decolagens em um sistema de pistas baseado em demanda contínua. A capacidade é estimada determinando-se o tempo mínimo de intervalo entre aproximações e invertendo-se esse tempo para encontrar o número máximo de aproximações por hora. A quantidade máxima de decolagens possíveis nos intervalos entre as aproximações é então, calculada (SWEDISH, 1981). Os modelos analíticos, em geral, são aplicados a componentes do sistema e não são tão precisos quanto os modelos de simulação, mas servem para obter resultados 
preliminares de capacidade rapidamente e também para estabelecer comparações iniciais entre diferentes sistemas de pistas.

- Simulações: Imitações do funcionamento de um sistema real mostrando sua evolução ao longo do tempo (WINSTON, 2004). Normalmente constituem-se de um software baseado em fórmulas matemáticas e restrições lógicas para possibilitar a imitação do funcionamento do sistema real, geralmente em tempo acelerado (fast time simulation). Baseados em dados e restrições de entrada, como volume e regras de tráfego aéreo, por exemplo, mostram resultados do funcionamento do sistema em métricas desejadas, como tempos de voo, atrasos, etc.

Os modelos de simulação possuem ainda outras características que permitem sua classificação em diferentes tipos. Do ponto de vista da representação no tempo, um modelo pode ser discreto ou contínuo. Modelo discreto é aquele onde suas variáveis mudam de valor em posições discretas ou contáveis do tempo. Modelo contínuo é aquele onde suas variáveis mudam de valor continuamente no tempo. Podem ser ainda classificados em estáticos e dinâmicos: modelos estáticos representam o sistema num particular instante definido e fixo do tempo. Já modelos dinâmicos são capazes de mostrar as modificações e evoluções do sistema ao longo de um período de tempo.

Considerando os valores que podem ser assumidos por suas variáveis, um modelo pode ser classificado em determinístico ou estocástico: modelo determinístico é aquele que não contém variáveis aleatórias e suas variáveis assumem sempre o mesmo valor quando diversas iterações são repetidas. Os modelos estocásticos possuem variáveis aleatórias e seus valores podem-se modificar em diferentes iterações, geralmente associados a distribuições de probabilidades.

\subsection{Software de Simulação}

Diversos software têm sido utilizados para construir modelos de simulação de aeroportos e de tráfego aéreo. Alguns são especificamente desenvolvidos para esse tipo de simulação, com regras de tráfego aéreo já pré-definidas e com interfaces gráficas que facilitam a modelagem de procedimentos de saída e chegada de 
aeronaves. Outros são software de simulação de uso geral, que permitem simulações de muitos tipos de sistemas, desde filas de pessoas até de aeronaves. A seguir são apresentadas as principais características de algumas dessas aplicações.

- ARENA - O ARENA é um software de simulação lançado em 1993 pela empresa Systems Modeling, tendo sido apresentado como sucessor de outras aplicações de simulação mais antigos da mesma empresa, o SIMAN e o CINEMA. A System Modeling foi posteriormente incorporada pela Rockwell Software, atual distribuidora do ARENA. O ARENA possui um conjunto de módulos que são utilizados para descrever um sistema real. Cada módulo possui comandos como os de uma linguagem de programação, adequados para a tarefa de simulação. Para simplificar a construção de modelos, o ARENA dispõe de uma interface gráfica. Além disso, fazem parte do software os módulos de input e output analyser. O Input Analyser permite analisar dados reais do funcionamento do sistema em estudo e escolher a melhor distribuição estatística que se aplica a eles. O Output Analyser permite analisar dados coletados durante a simulação de forma gráfica ou com comparações estatísticas (PRADO, 2003).

- SIMMOD - O SIMMOD, Airport and Airspace Simulation Model, foi desenvolvido pela FAA com o objetivo de ser uma ferramenta padrão de análise de cenários de tráfego aéreo. Trata-se de uma ferramenta capaz de simular operações de solo, bem como de espaços aéreos adjacentes aos aeroportos, onde se encontram os procedimentos de saída e chegada, ou espaços aéreos isolados. Trata-se de um modelo desenvolvido para responder a perguntas do tipo "What... If..." para diferentes configurações simuladas, auxiliando planejadores em tomadas de decisão. As principais medidas de desempenho apresentadas como resultado de simulação são tempos de voo e de atraso, consumo de combustível e capacidade de processamento de fluxo de tráfego em determinadas partes do espaço aéreo simulado. Inicialmente, tratava-se de uma ferramenta que operava somente em modo texto, obrigando os usuários a criar arquivos muito grandes com os parâmetros de entrada e a analisar inúmeros dados de saída para obter as respostas desejadas. Posteriormente, a empresa Airport Tools desenvolveu o 
Visual SIMMOD, um pacote completo de interface gráfica para ser usado em conjunto com o SIMMOD. Esse pacote facilita imensamente 0 trabalho de modelagem dos cenários e produz relatórios em formatos mais amigáveis para análise dos resultados das simulações (FAA, 2003; MOSER, 2007).

- RAMS Plus - O Reorganized ATC Mathematical Simulator (RAMS) foi desenvolvido pela empresa ISA Software em 1991, em cooperação com o EUROCONTROL, baseado num modelo chamado EAM (Eurocontrol Airspace Model), para a realização de estudos relacionados a ATM (Air Traffic Management). Trata-se de uma ferramenta de simulação fast time dedicada, capaz de modelar aeroportos e espaço aéreo. Recebe como dados de entrada as características dos aeroportos envolvidos, procedimentos de saída e chegada e regras de tráfego aéreo, através de uma interface gráfica que facilita a construção dos modelos. É considerado um simulador microscópico, voltado a análises detalhadas e projetos preliminares de aeroportos, e a configurações de espaços aéreos. Baseia-se na geração de trajetórias de voos, detecção e resolução de conflitos e violações de separações mínimas de tráfego aéreo, de acordo com regras pré-estabelecidas no modelo. Produz como resultados diversos relatórios numéricos e gráficos com os tempos de voo e atrasos (MOSER, 2007; POGIANELO, 2008; ISA, 2009).

- TAAM - O Total Airspace \& Airport Modeller (TAAM) é considerado uma das mais avançadas ferramentas de simulação aérea atualmente disponível. Foi desenvolvido pelo The Preston Group em cooperação com a Autoridade de Aviação Civil Australiana e, posteriormente, passou a pertencer à empresa Jeppesen, parte do grupo Boeing. Trata-se de um simulador dedicado a tráfego aéreo e aeroportos de larga escala, capaz de realizar modelagens fast time de sistemas complexos de tráfego aéreo e aeroportos, mostrando voos no formato conhecido como gate-to-gate, isto é, desde o estacionamento da aeronave antes da partida até o estacionamento após a chegada. É capaz de simular detalhadamente os diversos subsistemas aeroportuários, incluindo estacionamento, pistas de táxi, pistas de pouso, áreas de de-icing, baías de espera, etc. Pode também modelar procedimentos de saída e chegada, diferentes condições meteorológicas e regras de tráfego aéreo. O nível de 
detalhe dos dados de entrada é variável e pode ser adaptado de acordo com o objetivo do estudo a ser realizado. Permite a análise de tempos de voo, atrasos, carga de trabalho de controladores de tráfego aéreo, detecção e resolução de conflitos, impactos de mudanças de regras, impacto de ruídos nas proximidades de aeroportos e estudos de relações custo-benefício. Além de ser utilizado por entidades gestoras de tráfego aéreo, como FAA dos Estados Unidos, EUROCONTROL da Europa e DECEA no Brasil, pode ser usado por companhias aéreas para avaliar impactos de mudanças de frotas, melhorias em procedimentos operacionais, políticas de economia de combustível e impactos de modificações na circulação aérea determinadas pelas autoridades (SUBRAMANIAN, 2002; JEPPESEN, 2010a).

- REDIM - O Runway Exit Design Interactive Model é uma ferramenta de simulação desenvolvida pela Universidade Virginia Tech para auxiliar em estudos de localização e avaliação de saídas de pistas de pouso. Utiliza equações cinéticas, programação dinâmica polinomial e simulação de Monte Carlo para determinar a posição ótima das saídas de pista, considerando o conjunto de aeronaves que nela operam, ou para determinar o tempo de ocupação de pista por aeronaves em função de saídas já existentes (VIRGINIATECH, 2010).

Odoni et al. (1997) apresentam diversas outras aplicações de simulação que podem ser utilizados para modelagem de aeroportos e espaço aéreo. A seguir são compiladas as principais características de alguns dessas aplicações.

- The Airport Machine - É uma ferramenta desenvolvida pela Airport Simulation International para simulação detalhada de todos os aspectos de um aeroporto, incluindo pistas de pouso, de táxi e áreas de estacionamento. Sua principal medida de desempenho é o fluxo de aeronaves no aeroporto por unidade de tempo. É baseada numa estrutura de nós e arcos similar ao SIMMOD e cobre todas as atividades das aeronaves, desde alguns minutos antes do pouso até a alguns minutos após a decolagem. Possui uma boa interface gráfica, útil para calibração e validação do modelo. O pósprocessamento da simulação identifica fluxos e atrasos nos componentes, 
bem como possíveis gargalos do sistema. Os dados de saída incluem número de pousos e decolagens num intervalo de tempo, tempos de ocupação de posições de estacionamento, estatísticas de operações de push back, número de posições de estacionamento ocupadas e de aeronaves na fila para decolagem em cada instante de tempo.

- HERMES - HEuristic Runway Movement Event Simulation é uma ferramenta de simulação desenvolvida pela British Aviation Authority / National Air Traffic Services para avaliação da capacidade de pistas paralelas e de carga de trabalho de controladores de tráfego aéreo. Pode ser utilizada para avaliar capacidade de pistas e tempos de operação sob condições atuais e futuras de demanda, com a adoção de melhorias no sistema e modificações de infraestrutura. Utiliza dados experimentais de trajetórias de aeronaves como entrada e as principais saídas são medidas de atraso e estatísticas correspondentes.

- NASPAC - National Airspace System Performance Capability é um modelo de simulação fast time e macroscópico, desenvolvido pela MITRE Corporation nos anos 80 e mantido pelo Simulations and Analysis Group da FAA, que engloba grandes regiões do espaço aéreo e um grande número de aeroportos. O programa computa cada voo inserido nos itinerários diários, incluindo sequências de pousos e decolagens em diferentes aeroportos e fornece relatórios estatísticos de atrasos e fluxos de aeronaves observados.

- TMAC - Ferramenta de simulação desenvolvida pela MITRE Corporation. Trata-se de vários módulos compilados em conjunto, que utiliza planos de voo de aeronaves, dados de desempenho e estratégias de gerenciamento de tráfego aéreo para determinar conflitos e atrasos em um nível de detalhe maior que o NASPAC. É uma ferramenta de uso interno da MITRE e não está disponível externamente.

- FLOWSIM - Protótipo de ferramenta de simulação fast time da FAA que utiliza dados de planos de voo, desempenho padrão das aeronaves e fluxos de aeronaves entre grandes aeroportos para determinar atrasos e "efeitos de 
onda" induzidos por restrições de capacidade. A capacidade assumida dos aeroportos é fixa e baseada em sua configuração e meteorologia. Não é possível simular operações em rota. A simplicidade do modelo permite operação rápida (cerca de 5 minutos para simular um cenário), facilitando a avaliação de diversos cenários rapidamente, porém, de uma forma aproximada.

- ASCENT - ATFM System Concept Evaluator for New Technologies foi desenvolvido para avaliar o impacto global de novos procedimentos, tecnologias e melhorias de infra-estrutura sob condições existentes ou previstas de gerenciamento de fluxo de tráfego aéreo. Os voos previstos (demanda) e a capacidade dos aeroportos (oferta) são os fatores mais significativos na definição dos cenários. O modelo assume que as áreas de controle terminais são os pontos principais de congestionamento e detalha mais a simulação da operação nessas áreas em relação às áreas de rotas. $\mathrm{A}$ simulação fornece os horários planejados e realizados de cada voo durante a simulação e respectivas estatísticas, que podem ser filtradas de diversas formas: por companhia aérea, aeroporto, tipo de aeronave, período de tempo, etc.

\subsection{Trabalhos Relacionados}

Simulações computacionais têm sido utilizadas em diversos estudos relacionados a aeroportos e capacidades operacionais. Possibilitam a validação de um modelo proposto baseado em dados conhecidos de um sistema e, após isso, a criação de novos cenários, considerando mudanças de regras e práticas operacionais no sistema ou intervenções físicas, como obras de melhorias e/ou ampliação. Com os resultados das simulações é possível determinar o custo-benefício de cada cenário simulado, podendo-se, então, escolher o mais vantajoso para ser adotado.

$\mathrm{Na}$ última década, diversos trabalhos foram realizados utilizando ferramentas de simulação para analisar capacidade de aeroportos, adoção de novas tecnologias, procedimentos operacionais e carga de trabalho imposta a controladores de tráfego aéreo. 
Santana (2002) utilizou o software SIMMOD para desenvolver um modelo de operação do Aeroporto Internacional de São Paulo - Guarulhos, com ênfase na operação de solo - pátios de estacionamento, pistas de táxi e de pouso. Com o modelo desenvolvido, simulou os ganhos de capacidade com a construção de mais um terminal de passageiros - em função das posições de estacionamento correspondentes - e novas pistas de pouso e táxi, determinando a capacidade do aeroporto com essas intervenções, separadas e em conjunto.

Subramanian (2002) construiu um modelo de simulação do Philadelphia International Airport utilizando o software TAAM com vistas a determinar a capacidade operacional do aeroporto e consequentes níveis de atraso, bem como propor alternativas de melhorias, como a construção de uma nova pista de pouso paralela ou transversal às já existentes.

Hupalo (2003) criou um modelo da TMA de São Paulo/SP, utilizando o software SIMMOD, com o objetivo de analisar o fluxo de tráfego aéreo na TMA. Foram propostos cenários com a construção de uma nova pista de pouso de Guarulhos, a extinção de operação de aeronaves de pequeno porte (não comerciais) no Aeroporto de Congonhas e ainda a migração de uma parcela dos voos de Congonhas para Guarulhos.

Ribeiro (2003) desenvolveu um modelo no software ARENA para estudar a utilização do pátio do Aeroporto de Congonhas e o tempo de turn around das aeronaves que lá operam. O estudo visa a determinar a capacidade máxima do pátio, bem como oferecer subsídios para o balanceamento da oferta de serviços prestados às aeronaves estacionadas.

Gualda et al. (2005) utilizaram um modelo construído com o software ARENA para analisar a viabilidade de instalação de pontes de embarque no Aeroporto de Congonhas e o dimensionamento da frota de ônibus necessária ao atendimento das posições de estacionamento remotas, incluindo análise de custo-benefício das intervenções propostas. 
Moser (2007) desenvolveu um modelo do Aeroporto Internacional de São Paulo Guarulhos utilizando o software Visual SIMMOD para estudar os possíveis benefícios de obras de expansão no pátio de estacionamento e a construção de algumas pistas de táxi adicionais. Para isso, criou cenários de simulação capazes de determinar a capacidade horária de pousos e decolagens e os tempos de atraso para cada uma das configurações.

Oliveira (2007) construiu um modelo de simulação da TMA de Brasília/DF a fim de analisar a carga de trabalho dos controladores de tráfego aéreo, utilizando o software RAMS Plus. Tal análise visa a determinar a capacidade máxima de movimentos limitada pelo controle de tráfego aéreo, bem como sugerir a modificação da divisão de setores da TMA Brasília, para permitir maior capacidade de fluxo de aeronaves.

Bastos et al. (2008) desenvolveram um modelo utilizando o software ARENA para simular a ocupação das posições de estacionamento do Aeroporto de Congonhas pelas aeronaves, em vista de uma nova regulamentação criada pela ANAC. Os cenários simulados levaram em conta variações na separação entre as aeronaves em aproximação e o tempo de permanência no estacionamento, com o objetivo de determinar a capacidade real do pátio em cada situação.

Barragan e Alves (2008) utilizaram um modelo construído com o software ARENA para estudar a capacidade do Aeroporto Internacional de Campinas - Viracopos, bem como o impacto de modificações na proporção entre decolagens e pousos, na separação entre aeronaves em aproximação e da implantação de radar de aproximação na CTR (Control Zone) Campinas.

Müller e Santana (2008) utilizaram um modelo construído com o SIMMOD para analisar os atrasos dos voos na TMA de São Paulo e os custos decorrentes dos atrasos. Diversos cenários foram propostos, considerando a construção de mais um terminal de passageiros e mais uma pista de pouso no Aeroporto de Guarulhos, a remoção de aeronaves mais lentas de Congonhas e o remanejamento de parte do tráfego de Congonhas para Guarulhos, com vistas a reduzir os custos para as empresas aéreas. 
Pogianelo (2008) utilizou o software RAMS Plus para criar um modelo de simulação da TMA de Recife/PE. Com o modelo desenvolvido, foram simulados cenários com saídas e chegadas baseadas no sistema GNSS (Global Navigation Satellite System). Com os cenários simulados, buscou determinar níveis de atraso, carga de trabalho dos controladores de tráfego aéreo, bem como estimativa de economia de combustível por parte das aeronaves que operam no aeroporto.

Barragan (2009) utilizou o software ARENA para construir um modelo de simulação dos aeroportos existentes na CTR de São Paulo. Com o modelo construído, foram simulados diferentes cenários de uso dos aeroportos de Congonhas e Guarulhos, obtendo como resultado a capacidade de movimentos e melhorias possíveis com alterações de regras de tráfego aéreo, especialmente redução da separação nas aproximações e decolagens consecutivas.

Bastos (2009) construiu um modelo de simulação da TMA de Salvador/BA com o software RAMS Plus a fim de analisar os impactos da adoção de PBN nos procedimentos de saída e chegada daquela localidade. A análise dos resultados permitiu a avaliação de melhorias no fluxo de tráfego aéreo, capacidade do aeroporto e economia de combustível por parte das aeronaves em operação.

Baum (2009) analisou a carga de trabalho imposta aos controladores de tráfego aéreo da TMA de São Paulo por meio de um modelo de simulação desenvolvido com o software RAMS Plus. Tal análise visa a determinar a carga máxima de trabalho que pode ser imposta ao controlador de cada setor da TMA, bem como indicar possíveis modificações na estrutura do espaço aéreo, a fim de permitir um aumento da capacidade dos aeroportos envolvidos.

Fraga (2009) desenvolveu um modelo de simulação do Aeroporto Internacional de São Paulo - Guarulhos, utilizando o software RAMS Plus, para estudar a viabilidade e os possíveis benefícios da implantação do sistema PRM/SOIA (Precision Runway Monitor / Simultaneous Offset Instrument Approach) para aproximação, verificando os consequentes níveis de atraso para decolagens e pousos com a utilização do novo sistema em relação ao atual. 


\subsection{Conclusões do Capítulo}

Nesse capítulo foi apresentada uma revisão da literatura, que inclui os conceitos necessários para o desenvolvimento do trabalho e uma revisão de outros trabalhos existentes a esse respeito.

O presente trabalho difere dos acima apresentados, pois pretende avaliar a capacidade do lado aéreo de aeroportos sem intervenções físicas ou a criação de procedimentos baseados em novas tecnologias. As variações propostas baseiam-se na modificação de procedimentos utilizados por controladores de tráfego aéreo, respeitando-se critérios de nível de serviço e recomendações da ICAO. Tais modificações, por não envolverem obras ou aquisição de equipamentos, podem ser implementadas com baixo custo e em curto espaço de tempo.

No capítulo seguinte é apresentada uma revisão de conceitos de espaço aéreo e regras de tráfego aéreo. 


\section{ESPAÇO AÉREO}

Neste capítulo é apresentado um resumo das regras de tráfego aéreo e separação de aeronaves que afetam as operações de decolagem, subida, aproximação e pouso. Tais regras são importantes por influenciar diretamente a capacidade de operação do lado aéreo dos aeroportos.

Após a Convenção de Aviação Civil ocorrida em Chicago, Estados Unidos, em 7 de dezembro de 1944 (conhecida como Convenção de Chicago), a ICAO passou a emitir recomendações para os países signatários a respeito de diversos assuntos relacionados à aviação civil, visando a promover o desenvolvimento seguro e ordenado do transporte aéreo internacional (ICAO, 2006).

No tocante a regras e serviços de tráfego aéreo, as recomendações são dadas pelo Annex 2 - Rules of the Air (ICAO, 2005), Annex 11 - Air Traffic Services (ICAO, 2001), Doc 4444 - Air Traffic Management (ICAO, 2007) e Doc 7030 - Regional Supplementary Procedures (ICAO, 2008). No Brasil, os procedimentos recomendados pela ICAO são adotados pelo Comando da Aeronáutica e publicados pelo DECEA. As regras estão, em sua maioria, contidas na ICA 100-12 - Regras do Ar e Serviços de Tráfego Aéreo (COMANDO DA AERONÁUTICA, 2009) e devem ser do conhecimento de pilotos e controladores de tráfego aéreo.

\subsection{Espaço Aéreo Brasileiro}

O espaço aéreo sob jurisdição do Brasil, além daquele que superpõe o território brasileiro e as águas territoriais, estende-se até o meridiano 10W, conforme Acordo Regional de Navegação Aérea específico. Esse espaço aéreo é, então, dividido em áreas de informação de voo e de controle de tráfego aéreo, de acordo com a densidade de tráfego e serviços necessários.

Para um melhor entendimento do espaço aéreo brasileiro, faz-se necessário apresentar as seguintes definições (COMANDO DA AERONÁUTICA, 2009b): 
Espaço Aéreo Inferior - Espaço aéreo compreendido entre o solo ou água e o nível de voo 245 (24.500 pés), inclusive.

Espaço Aéreo Superior - Espaço aéreo que possui como limite inferior o nível de voo 245 , exclusive, e não possui limite superior (ilimitado).

Região de Informação de Voo (FIR) - Espaço aéreo onde é prestado o serviço de informação de voo e alerta. Corresponde à maior parte do espaço aéreo brasileiro. Quando esta região de informação estiver no espaço aéreo superior, será denominada Região Superior de Informação de Voo (UIR), porém, quando os limites laterais da UIR coincidirem com os da FIR inferior, ambos serão chamados de FIR. Existem atualmente no Brasil 5 FIR, conforme ilustrado na figura 4.

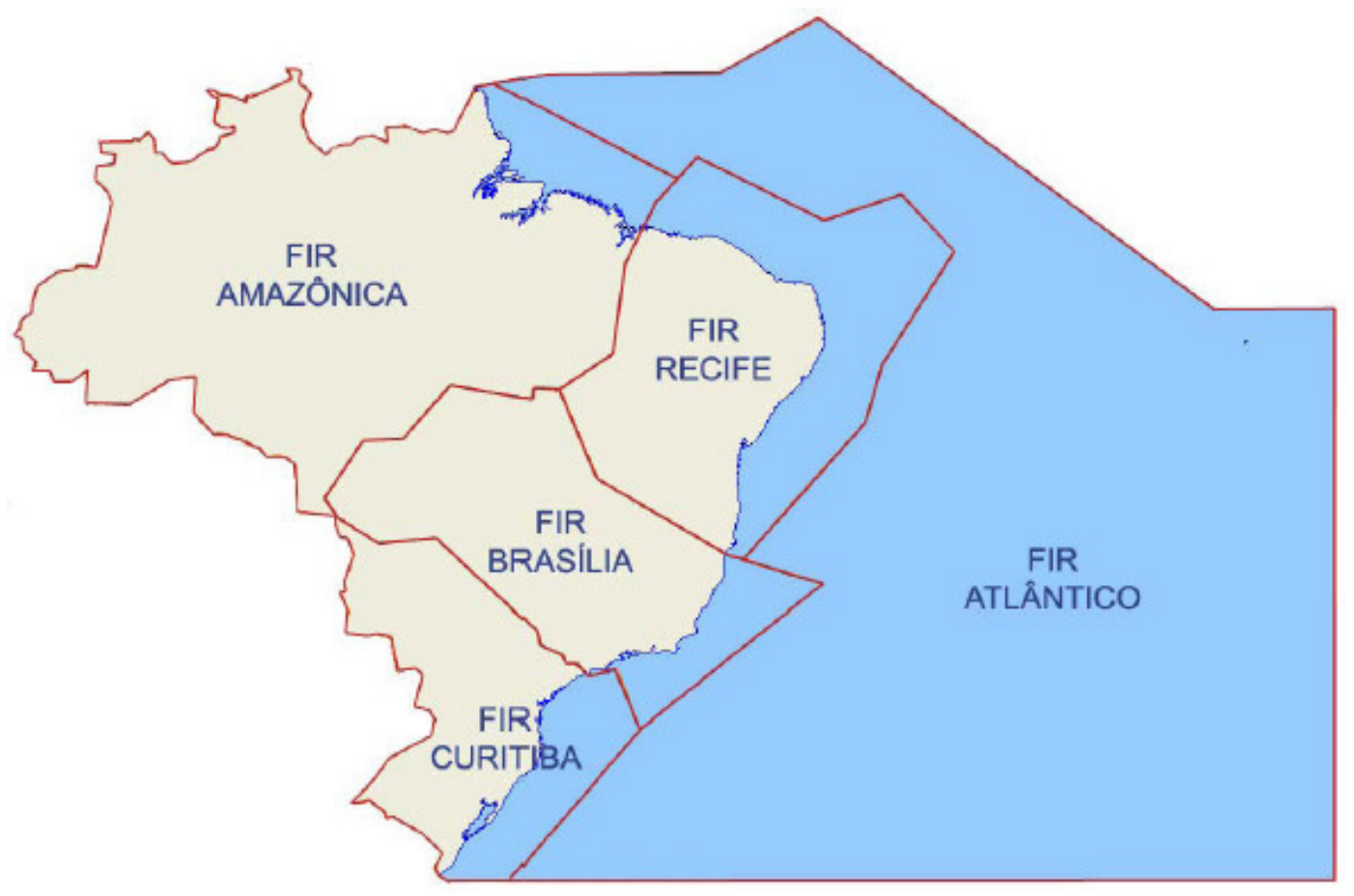

Figura 4 - Regiões de Informação de Voo do Brasil Fonte: (COMANDO DA AERONÁUTICA, 2009a)

Aerovia (AWY) - Área de controle disposta em forma de corredor e provida de auxílios rádio a navegação. Sua finalidade é ordenar o tráfego aéreo, permitir a prestação de serviços de controle e o gerenciamento do fluxo deslocando-se em rota. 
Regras de Voo Visual (VFR) - Quando operando em voo visual, o piloto deve manter referências visuais com o solo e distâncias mínimas de nuvens que variam de acordo com o tipo de espaço aéreo onde se encontra.

Regras de Voo por Instrumentos (IFR) - Quando operando em voo por instrumentos, o piloto não necessita de visibilidade externa, podendo operar dentro de nuvens e em condições de visibilidade reduzida, sem referências externas. A condução do voo é feita exclusivamente por meio das informações dos instrumentos de bordo.

Área de Controle Terminal (TMA) - Área geralmente situada na confluência de rotas ATS e nas imediações de um ou mais aeródromos importantes. Tem como objetivo controlar e proteger as aeronaves durante os procedimentos de subida (SID) e chegada (STAR). Os limites laterais e verticais variam de acordo com a área e são indicados nas cartas de navegação.

Zona de Controle (CTR) - Espaço aéreo controlado, cujo objetivo principal é proteger os procedimentos IFR de saída ou descida. A CTR tem configuração variável e estende-se do solo ou água até um limite vertical especificado nas cartas de navegação.

Zona de Tráfego de Aeródromo (ATZ) - É o espaço aéreo controlado cujo objetivo é proteger o circuito de tráfego do aeródromo, onde encontram-se as aeronaves saindo e chegando sob regras de voo VFR. A configuração da ATZ é variável e, nas localidades onde o circuito de tráfego difere do padrão estabelecido nas regras do ar, os limites da ATZ são representados na carta de aproximação visual.

\subsection{Classificação do Espaço Aéreo}

De acordo com o tipo de serviço prestado, os espaços aéreos são classificados alfabeticamente, com letras que vão de "A" até "G". Para cada tipo de espaço aéreo são definidas as regras de operação, o tipo de serviço prestado pelos órgãos de controle, os mínimos de separação entre as aeronaves, os limites de velocidade, os equipamentos de comunicação necessários e a exigência ou não de autorização de 
tráfego aéreo. A figura 5 ilustra a classificação dos espaços aéreos. São descritos os tipos de separação e serviços prestados pelos órgãos de controle de tráfego aéreo, as distâncias mínimas para as nuvens e visibilidade requerida (apenas para voos VFR), os limites de velocidade aplicáveis às aeronaves, a obrigatoriedade ou não de a aeronave possuir equipamento de rádio comunicação e a necessidade de autorização prévia de um órgão de controle de tráfego aéreo para a realização do voo.

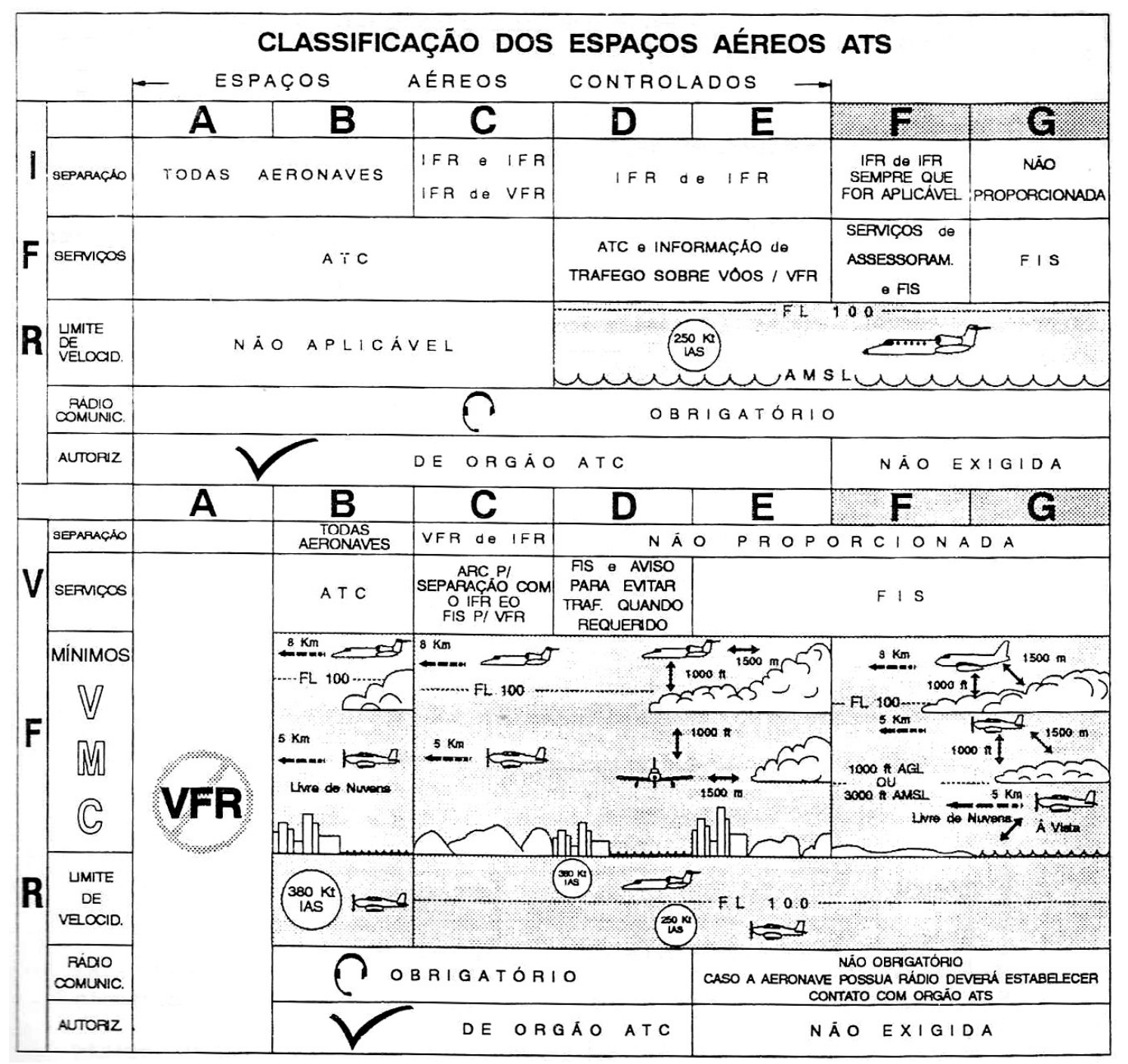

Figura 5 - Classificação dos espaços aéreos ATS Fonte: (SOARES, 1993)

Classe "A" é a classificação dada ao espaço aéreo superior, onde todos os voos recebem o serviço de controle de tráfego aéreo, devendo receber autorização de um órgão ATC, que é responsável pela separação entre as aeronaves. 
Não existem espaços aéreos classe "B" no Brasil.

São classe "C" os espaços aéreos que constituem as áreas de controle terminal com cobertura radar. Nessas áreas, todos os voos IFR são controlados e separados dos VFR. Já os espaços aéreos classe "D" são as áreas de controle terminais que não dispõem de equipamento radar. O órgão de controle é, então, responsável apenas pela separação dos voos IFR e os VFR recebem serviços de informação de voo e avisos para evitar interferência com os voos IFR. Normalmente, são classe "D" as TMA de menor fluxo de tráfego.

Não existem espaços aéreos classe "E" e "F" no Brasil.

A classe "G" é reservada às regiões de informação de voo, que são grandes áreas onde não é prestado serviço de controle de tráfego aéreo e cada aeronave é responsável por prover sua separação em relação às demais. É prestado apenas o serviço de informação de voo, mas não é exigida autorização de órgão ATC.

\subsection{Regras de Tráfego Aéreo}

Os voos evoluindo numa área de controle terminal devem respeitar regras de separação entre as aeronaves, a fim de preservar a segurança. Num espaço aéreo classe C, como é o caso da TMA São Paulo, o controle é feito com cobertura de radar de aproximação e o controlador de tráfego aéreo é responsável pela separação das aeronaves, devendo emitir autorizações específicas para cada uma, de forma que a separação lateral e/ou vertical esteja assegurada.

\subsubsection{Separação Radar}

Quando sob cobertura radar, a separação vertical mínima é de 1000 pés (300 metros) e a lateral é de 5NM (milhas náuticas) para aeronaves operando segundo regras IFR. No espaço aéreo brasileiro, a separação lateral pode ser reduzida para até 3NM, desde que (COMANDO DA AERONÁUTICA, 2007):

- As aeronaves envolvidas estejam a menos de 40NM da antena radar; 
- As informações de posição das aeronaves sejam provenientes de radar primário, isto é, independente de equipamentos instalados a bordo das aeronaves;

- As informações sejam provenientes de uma única antena, não sendo permitido o uso de sistema multirradar;

- Os controladores de tráfego aéreo e os pilotos estejam familiarizados com esse procedimento.

Quando existir uma aeronave em aproximação e outra decolando, a separação deverá ser de pelo menos 3NM, considerando que a aeronave que decola deverá ter ultrapassado o final da pista.

Para separação entre decolagens, a ICAO (2007) recomenda um mínimo de:

- 1 minuto de intervalo se as aeronaves seguirem em rumos divergentes, pelo menos de 45 graus, como mostrado na figura 6 ;

- 2 minutos de intervalo se as aeronaves seguirem no mesmo rumo, desde que a aeronave precedente seja pelo menos 40 nós $(74 \mathrm{~km} / \mathrm{h})$ mais rápida, como mostrado na figura 6 ;

- 5 minutos de separação se a aeronave posterior for cruzar em altitude com a aeronave precedente seguindo a mesma rota de saída, como mostrado na figura 7 ;

Além dos mínimos de separação apresentados, a separação mínima relacionada à esteira de turbulência deverá ser aplicada sempre que for superior aos valores aqui descritos e o controlador de tráfego aéreo julgar necessário. Os procedimentos relacionados à esteira de turbulência são descritos na seção 3.4 . 

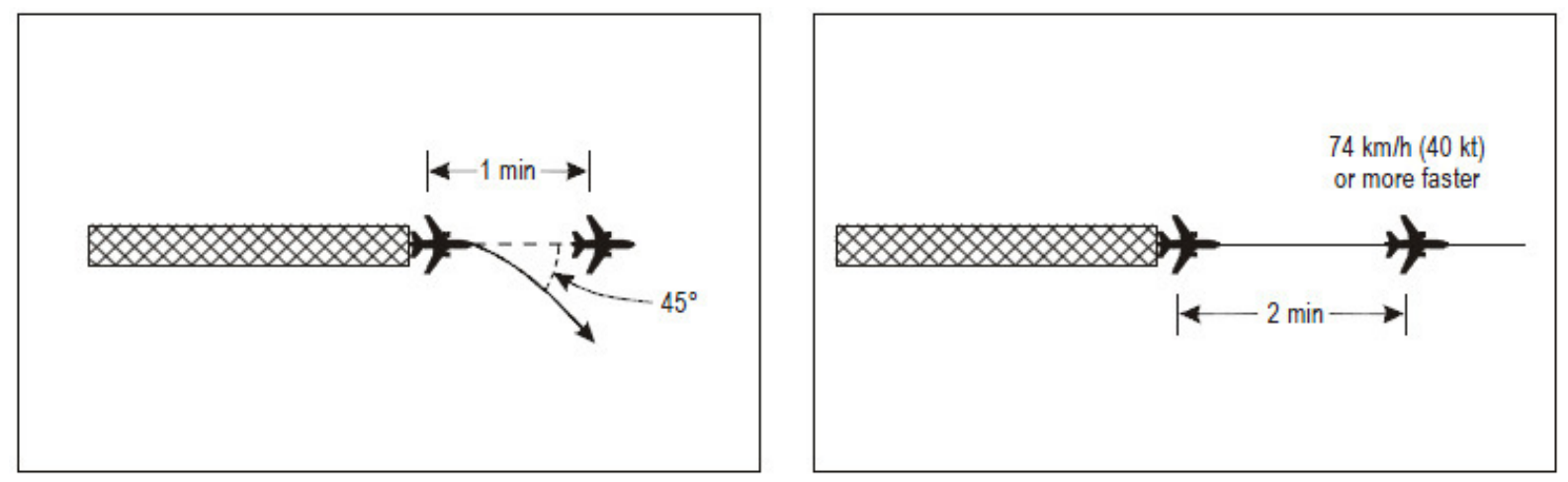

Figura 6 - Separação mínima entre decolagens

Fonte: (ICAO, 2007)

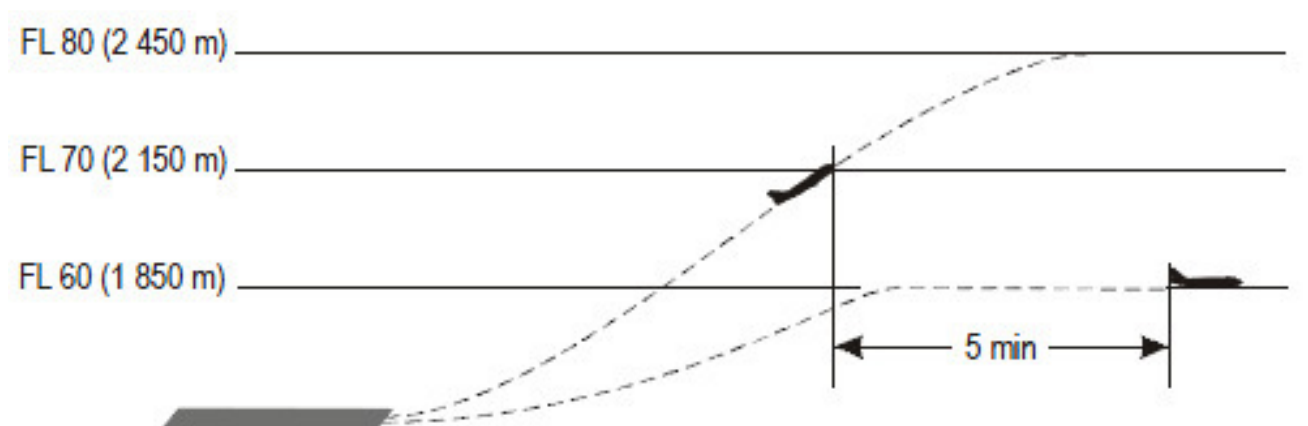

Figura 7 - Separação mínima entre decolagens com cruzamento em altitude Fonte: (ICAO, 2007)

\subsubsection{Serviços ATS}

Quando em espaço aéreo controlado com cobertura radar, as aeronaves em voo IFR poderão receber dois tipos de serviço de tráfego aéreo, a saber:

- Vigilância radar: Serviço onde a aeronave é identificada pelo radar de vigilância e passa a ter sua trajetória monitorada pelo controlador de tráfego aéreo. O piloto é o responsável pela navegação da aeronave.

- Vetoração radar: Serviço onde o controlador de tráfego aéreo é responsável pela navegação e separação da aeronave em relação a outras aeronaves e obstáculos, devendo emitir autorizações de proa e altitudes específicas que serão seguidas pelo piloto.

No Brasil, os procedimentos SID baseiam-se exclusivamente na utilização de auxílios rádio à navegação ou em informações provenientes de sistema GNSS. Nesses procedimentos, os pilotos devem seguir trajetórias pré-estabelecidas nas cartas, recebendo, quando disponível, o serviço de vigilância radar. Exemplos de 
cartas SID encontram-se no Anexo A, figuras 30 a 32. Não existem procedimentos de subida baseados em vetoração radar no Brasil.

Já outros países com grande volume de tráfego aéreo, como os Estados Unidos, por exemplo, empregam procedimentos SID baseados em vetoração radar. Tais procedimentos visam agilizar o fluxo de tráfego aéreo e elevar a capacidade do sistema, porém, aumentam a carga de trabalho dos controladores de tráfego aéreo.

\subsection{Esteira de Turbulência}

Esteira de turbulência é um fenômeno que ocorre em função da sustentação da asa das aeronaves. As altas pressões existentes no intra-dorso fluem pelas pontas da asa em direção ao extradorso. Com o deslocamento longitudinal da aeronave, formam-se vórtices contrarrotativos atrás da aeronave. O tamanho e a intensidade dos vórtices estão relacionados ao peso e à envergadura da aeronave (FAA, 1995). A figura 8 ilustra a formação de vórtices por uma aeronave.

Os vórtices gerados por uma aeronave são potencialmente perigosos para outras aeronaves que vêm atrás, causando esforços estruturais, fortes tendências de rolamento, perdas de altitude e velocidade. Esse perigo é agravado quando as aeronaves estão a baixa altura, pois, nessa situação, sua manobrabilidade é limitada e há risco de colisão com o solo.

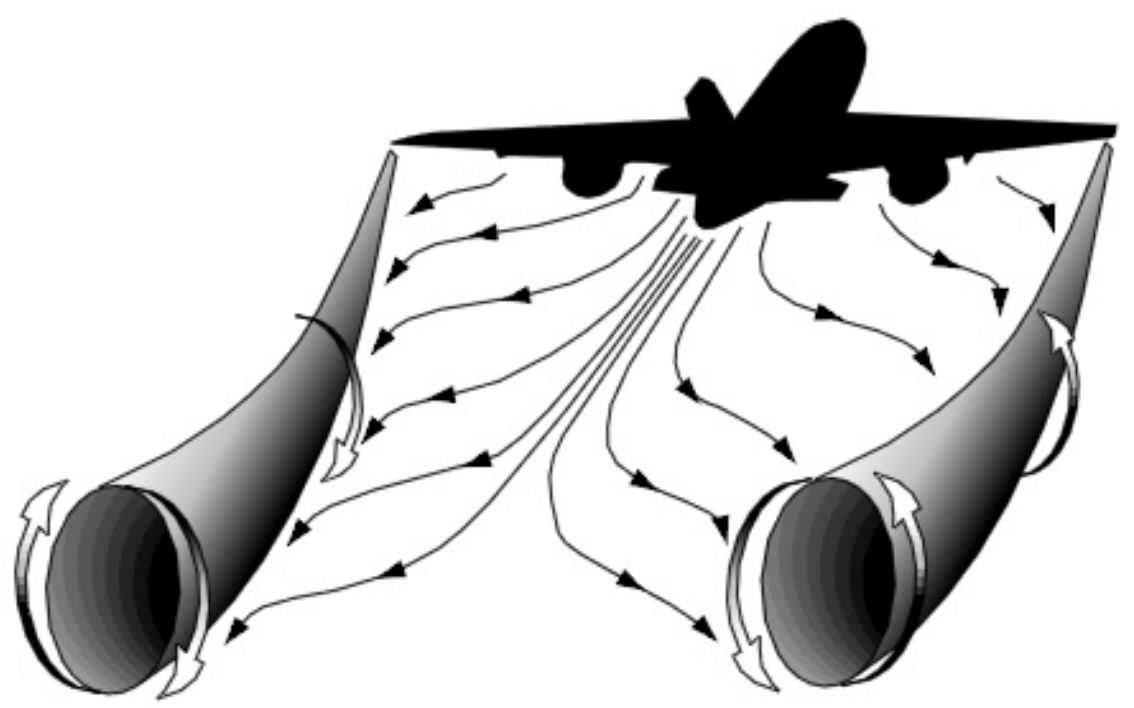

Figura 8 - Esteira de turbulência Fonte: FAA (1995) 


\subsubsection{Classificação das Aeronaves}

Para evitar-se os perigos oriundos da esteira de turbulência, faz-se necessário separar as aeronaves em função desse fenômeno. Para tanto, as aeronaves são divididas em três categorias, de acordo com o peso máximo de decolagem (COMANDO DA AERONÁUTICA, 2009b):

- Categoria Leve: aeronaves até $7000 \mathrm{~kg}$;

- Categoria Média: aeronaves com mais de 7000 e menos de $136.000 \mathrm{~kg}$;

- Categoria Pesada: aeronaves com $136.000 \mathrm{~kg}$ ou mais.

Com o surgimento das aeronaves Airbus A380-800, que têm peso máximo de decolagem de cerca de $560.000 \mathrm{~kg}$, um tratamento especial quanto à esteira de turbulência foi criado para esse modelo de aeronave (DECEA, 2008).

\subsubsection{Separação na Aproximação}

Sempre que o controlador de tráfego aéreo observar que existe um possível perigo devido à esteira de turbulência para aeronaves em aproximação, deverá aplicar a separação mostrada na tabela 2.

Tabela 2 - Mínimos de separação de esteira de turbulência Fonte: (COMANDO DA AERONÁUTICA, 2009b)

\begin{tabular}{ccc}
\hline $\begin{array}{c}\text { Categoria da aeronave } \\
\text { que segue à frente }\end{array}$ & $\begin{array}{c}\text { Categoria da aeronave } \\
\text { que segue atrás }\end{array}$ & Mínimos \\
\hline A380-800 ou outra & A380-800 & não requerido \\
aeronave pesada & Pesada & $6 \mathrm{NM}$ \\
A380-800 & Média & $7 \mathrm{NM}$ \\
& Leve & $8 \mathrm{NM}$ \\
\hline \multirow{3}{*}{ Pesada } & Pesada / A380-800 & $4 \mathrm{NM}$ \\
& Média & $5 \mathrm{NM}$ \\
& Leve & $6 \mathrm{NM}$ \\
\hline \multirow{2}{*}{ Média } & Pesada / A380-800 & $3 \mathrm{NM}$ \\
& Média & $3 \mathrm{NM}$ \\
& Leve & $5 \mathrm{NM}$ \\
\hline \multirow{2}{*}{ Leve } & Pesada / A380-800 & $3 \mathrm{NM}$ \\
& Média & $3 \mathrm{NM}$ \\
& Leve & $3 \mathrm{NM}$ \\
\hline
\end{tabular}




\subsubsection{Separação na Decolagem}

A ICAO recomenda que seja empregada uma separação mínima de 2 minutos quando uma aeronave leve ou média decolar atrás de uma pesada, ou quando uma aeronave leve decolar atrás de uma média, sempre que as aeronaves utilizarem a mesma pista ou pistas paralelas separadas por menos de 760 metros. Quando a decolagem da segunda aeronave ocorrer a partir de um ponto intermediário da pista, essa separação deverá ser aumentada para 3 minutos. As figuras 9 e 10 mostram a separação recomendada na decolagem para esses casos (ICAO, 2007).

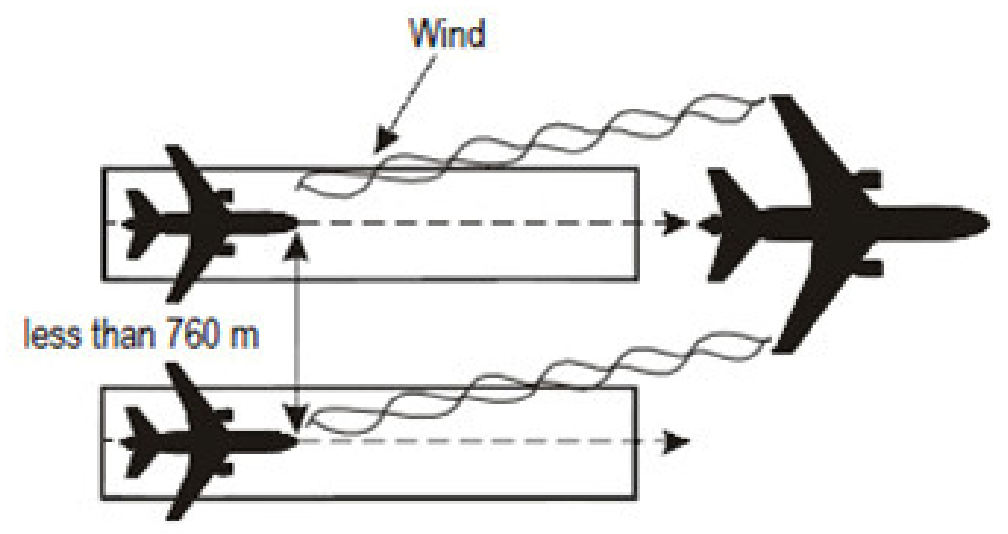

Figura 9 - Separação entre decolagens - 2 minutos Fonte (ICAO, 2007)

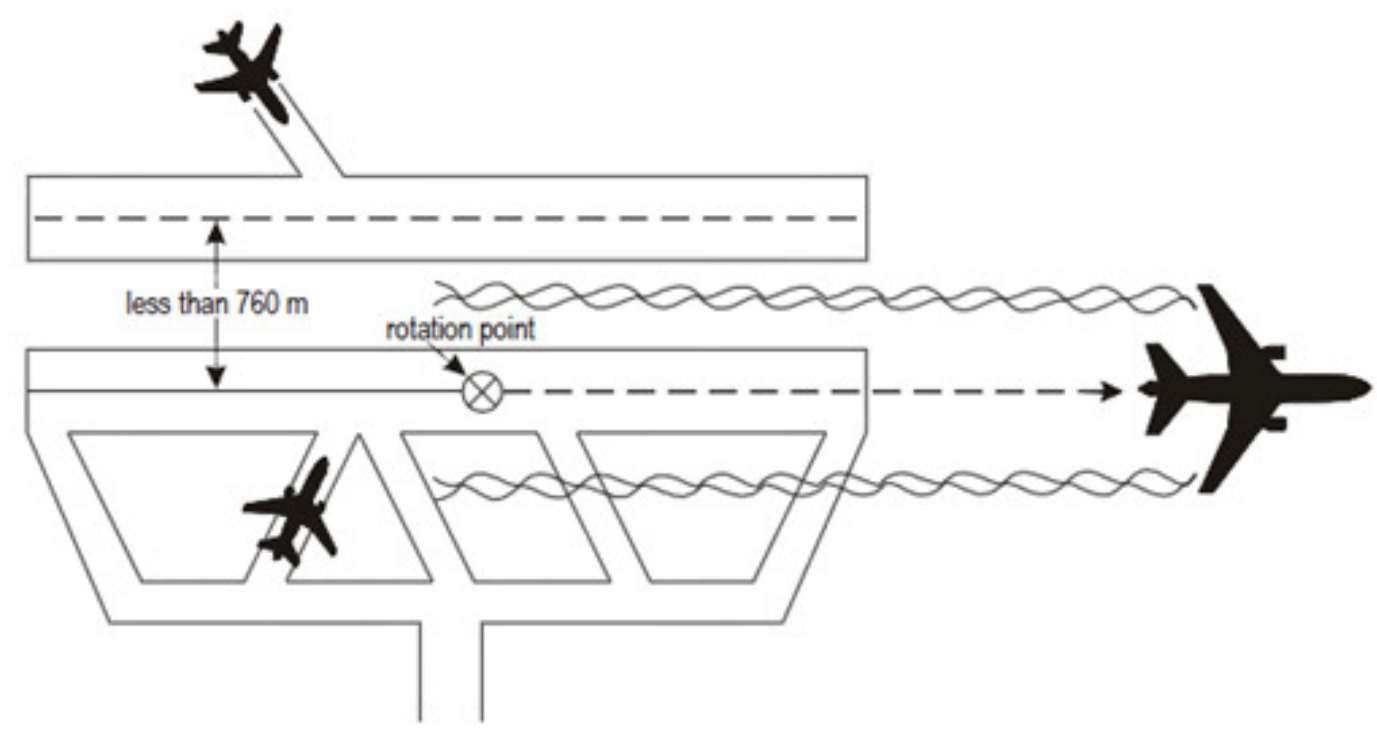

Figura 10 - Separação entre decolagens de pontos intermediários da pista - 3 minutos Fonte: (ICAO, 2007) 


\subsection{Conclusões do Capítulo}

Nesse capítulo foi apresentada uma breve descrição das regras de tráfego aéreo que afetam a operação de aeronaves nas proximidades dos aeroportos, principalmente aquelas realizando procedimentos de saída e chegada. No capítulo seguinte é apresentada a metodologia proposta para análise de capacidade do lado aéreo de aeroportos, considerando as regras aqui apresentadas. 


\section{METODOLOGIA}

A metodologia proposta nesse trabalho consiste na aplicação do método científico combinado ao enfoque sistêmico, já apresentado na seção 2.1. Gualda (1995) considera as seguintes etapas para a utilização desse método:

1. Identificação do sistema;

2. Formulação do problema e das medidas de rendimento;

3. Geração de alternativas para solução do problema;

4. Avaliação das alternativas;

5. Seleção da alternativa que melhor atende às premissas consideradas.

A seguir, são descritos os passos necessários para implementação dessas etapas.

\subsection{Identificação do Sistema}

Considerando as definições de enfoque sistêmico descritas na seção 2.1, o sistema que será analisado é o lado aéreo do aeroporto, composto pelas posições de estacionamento, pistas de táxi, pistas de pouso e espaço aéreo da área terminal ao redor do aeroporto.

\subsection{Formulação do Problema}

$\mathrm{Na}$ avaliação do lado aéreo de um aeroporto, deve-se, então, identificar e caracterizar cada um dos componentes que se deseja avaliar, para inserção no modelo. O levantamento de dados deve incluir os seguintes itens:

- Pistas de pouso: Deve-se realizar um levantamento das características físicas das pistas de pouso, incluindo dados como: comprimento, largura, distância entre pistas, posição e tipos das saídas, resistência do pavimento, etc. É importante também obter informações sobre os ventos predominantes no aeroporto, a fim de determinar qual a cabeceira mais utilizada. 
- Pistas de táxi: As pistas de táxi devem ser igualmente caracterizadas, com atenção a eventuais restrições de operação de determinados modelos de aeronave.

- Pátios de estacionamento: Deve-se conhecer a quantidade de posições de parada, os tipos de aeronave que podem ocupar cada uma delas e os tempos médios de permanência de cada tipo de aeronave nas posições.

- Espaço aéreo: Com relação ao espaço aéreo adjacente ao aeroporto, é necessário conhecer os procedimentos de saída (SID) e chegada (STAR) utilizados, incluindo suas trajetórias e restrições operacionais. Regras de tráfego aéreo e procedimentos operacionais específicos do aeroporto, se houver, devem também ser considerados, com ênfase nas separações de saída e chegada utilizadas pelos controladores de tráfego aéreo.

- Desempenho das aeronaves: É necessário dispor de dados de desempenho dos modelos de aeronave envolvidos, tais como: velocidades de saída e de aproximação, altitudes em função da distância percorrida, tempos de ocupação de pista, velocidades de táxi, etc.

- Conjunto de voos: É necessário definir um conjunto de voos que será utilizado, com base num dia considerado de maior movimento na operação do aeroporto (dia pico), de acordo com o cenário que se deseja modelar.

\subsection{Medidas de Rendimento Consideradas}

A escala de nível de serviço apresentada na seção 2.2 considera que um aeroporto opera de forma aceitável quando o nível de atraso médio das aeronaves é igual ou inferior a cinco minutos. Para a determinação de capacidade, será encontrado o número máximo de movimentos que proporcione um atraso médio aceitável para aeronaves na fila de decolagem. Para a execução das simulações, os tempos de atraso para decolagem serão calculados como o tempo de atraso ainda na posição de estacionamento (tempo que a aeronave, quando já pronta para iniciar a partida, 
fica estacionada, aguardando autorização do controle de tráfego aéreo), somado ao tempo dilatado de táxi (tempo de táxi que excede a média para operações em horários não congestionados) e ao tempo de espera na fila de decolagem.

\subsection{Construção do Modelo de Simulação}

Tendo sido realizado o levantamento de todos os dados necessários, apresentados em 4.2, cria-se um modelo do lado aéreo do aeroporto em estudo, utilizando um software de simulação. A escolha do software deve levar em consideração fatores como disponibilidade de licença de uso, documentação existente e existência de outros trabalhos na literatura já realizados com o software. Considerando esses fatores, foi escolhido o software Visual SIMMOD para a realização deste trabalho. $O$ funcionamento do SIMMOD está descrito no apêndice $A$.

O SIMMOD requer definições de velocidade e altitude para cada segmento de rota criado. Para as rotas de chegada, são consideradas as altitudes mínimas e velocidades máximas apresentadas nas cartas. Para as altitudes nas rotas de saída, foram usados os dados da pesquisa de Hupalo (2003), que determinou as altitudes que as aeronaves atingem na subida, em função da distância desde o aeroporto de decolagem. Foram utilizadas as velocidades de subida características de cada tipo de aeronave, conforme a tabela de dados do SIMMOD.

Em relação à demanda, é recomendado que se utilizem dados de um dia pico para realizar as simulações, a fim de que seja possível observar os atrasos decorrentes da sobrecarga aplicada ao sistema. Para a escolha do dia pico, encontram-se na literatura algumas variações: Santana (2002) identificou o mês com maior número de movimentos dentro do período de estudo e, dentro deste, o dia com maior movimentação; Hupalo (2003) considerou, em seu trabalho, um dia com registro de movimentos acima do normal e para o qual havia um outro estudo que serviria como base de comparação; Moser (2007) escolheu um dia de movimento intenso para o qual também existia um outro trabalho de simulação que poderia ser comparado; Barragan (2009) selecionou o dia com maior número de movimentos no período estudado. 


\subsubsection{Replicações}

Em função da natureza estocástica do modelo de simulação, é necessário utilizar uma quantidade suficiente de iterações para obter resultados confiáveis. Seguindo as recomendações da literatura, serão realizadas 10 iterações de cada cenário, valor considerado adequado para utilização do SIMMOD (SANTANA, 2002 e MOSER, 2007).

\subsubsection{Validação do modelo}

Antes de utilizar um modelo para simular condições diferentes da realidade, é necessário certificar-se de que este retrata, de forma fiel, as condições reais de operação do sistema. Uma forma de validar um modelo é analisar seu comportamento em relação à operação real do sistema, comparando-se os resultados da simulação com dados obtidos na operação real num dia de referência, a fim de identificar erros de modelagem e/ou de funcionamento do software de simulação. A validação do modelo conta com os seguintes passos:

- Utilização da ferramenta Animator para verificação gráfica e visual da operação das aeronaves, com vistas a detectar erros de modelagem de procedimentos, conflitos de tráfego e gridlocks ${ }^{1}$;

- Análise dos dados quantitativos, onde são comparados os movimentos previstos e os efetivamente realizados pela simulação a cada hora, no período de funcionamento do aeroporto.

\subsection{Geração de Alternativas para Solução do Problema}

Com o modelo testado e considerado confiável, pode-se partir para a simulação dos diferentes cenários que se deseja avaliar. Não serão simulados cenários que envolvam modificações físicas no aeroporto, como a construção de novas pistas de pouso e de táxi, ou de novas posições de estacionamento, pois o objetivo do

\footnotetext{
${ }^{1}$ Gridlocks são conflitos entre aeronaves disputando um mesmo recurso do modelo, causando a interrupção da simulação.
} 
trabalho concentra-se em determinar a capacidade da infra-estrutura já instalada do aeroporto.

Para tanto, serão construídos os seguintes cenários:

- Operação atual do aeroporto;

- Variação na separação entre decolagens;

- Variação na separação entre aproximações;

- Combinação das variações nas separações entre decolagens e aproximações;

- Substituição de aeronaves mais lentas por outras mais velozes;

- Combinação da substituição de aeronaves com as variações de separações;

- Redistribuição dos voos ao longo do tempo.

\subsubsection{Variações de demanda}

Para todos os cenários simulados, serão aplicadas variações de demanda que vão desde $15 \%$ de redução até $15 \%$ de acréscimo no volume de voos regulares, em intervalos de $5 \%$. Tais variações têm o objetivo de permitir a análise do comportamento do sistema nos cenários simulados, bem como possibilitar a comparação dos resultados com a estimativa dos modelos teóricos.

\subsection{Avaliação e Seleção das Alternativas}

De posse dos resultados das simulações dos diferentes cenários, é possível discutir quais as alternativas mais viáveis para que o aeroporto possa oferecer o máximo de capacidade sem, no entanto, violar os níveis de serviço pré-estabelecidos para a operação.

\subsection{Conclusões do Capítulo}

Esse capítulo descreve a metodologia proposta para análise de capacidade do lado aéreo de aeroportos, que associa conceitos do método científico, de abordagem sistêmica e de capacidade associada a nível de serviço para determinar a 
capacidade e o nível de serviço oferecido às aeronaves. No próximo capítulo é mostrada a aplicação dessa metodologia ao Aeroporto de Congonhas. 


\section{APLICAÇÃO AO AEROPORTO DE CONGONHAS}

O Aeroporto de Congonhas fica localizado na região central da cidade de São Paulo, ocupando uma área de $1.647 .000 \mathrm{~m}^{2}$. Inaugurado oficialmente em 1936, em 1957 figurava como o terceiro aeroporto do mundo em volume de carga aérea. A partir de 1981, passou a ser administrado pela INFRAERO. (INFRAERO, 2010a). A figura 11 mostra a localização do aeroporto na cidade.

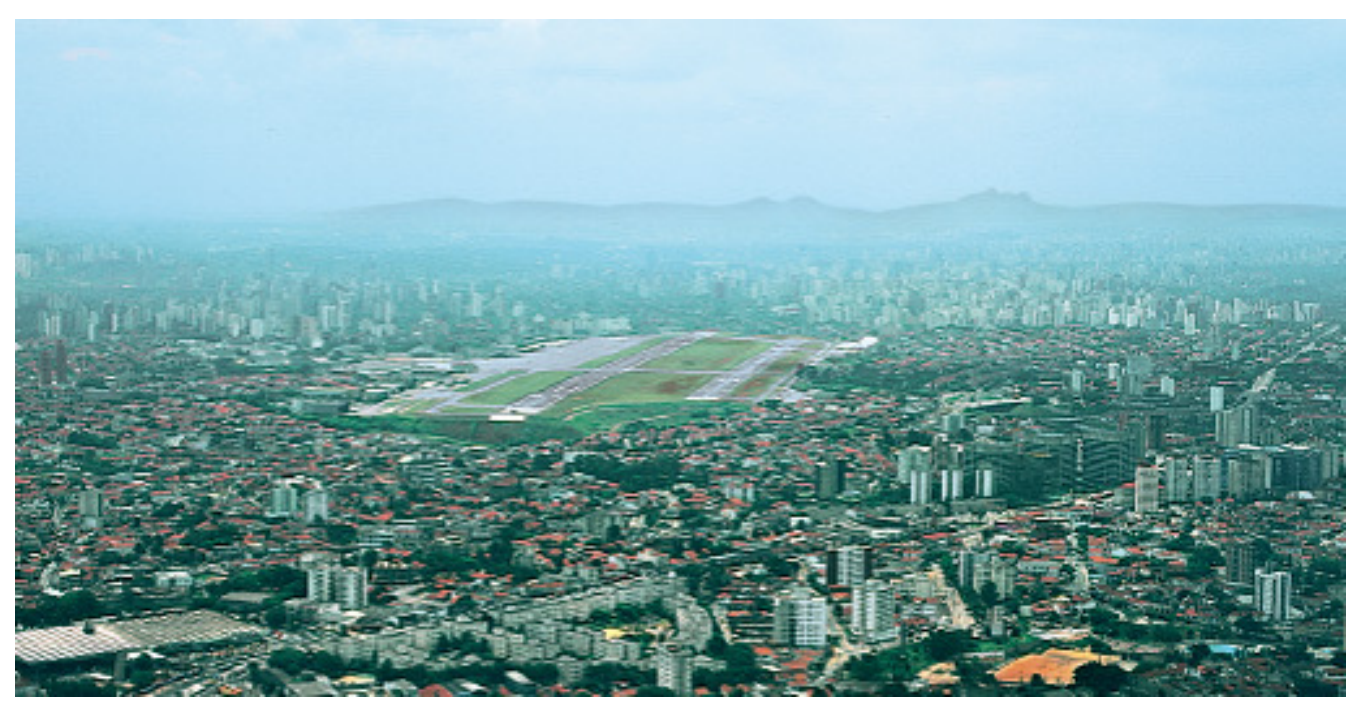

Figura 11 - Localização do Aeroporto de Congonhas Fonte: (JEPPESEN, 2010b)

Atualmente, Congonhas é um dos aeroportos mais movimentados do Brasil, tendo registrado em 2009 um total de 193.308 movimentos de decolagem e pouso, que transportaram cerca de 13.700 .000 passageiros. Segundo estudos realizados, a demanda em Congonhas cresce $5,6 \%$ ao ano e poderia chegar a 727.000 movimentos e quase 56 milhões de passageiros transportados em 2025, caso existisse capacidade instalada para suportá-la (COMANDO DA AERONÁUTICA, 2005).

Em contrapartida, a ANAC vem adotando medidas de restrição de tráfego cada vez mais severas em Congonhas, tais como: alocação de slots para operações, proibição de operação de aeronaves comerciais na pista auxiliar e determinação de tempo máximo de permanência de aeronaves nos boxes de estacionamento, entre outras (ANAC, 2006). Com isso, o número de movimentos nos últimos anos encontra-se muito abaixo do previsto, como pode ser visto na figura 12 . 


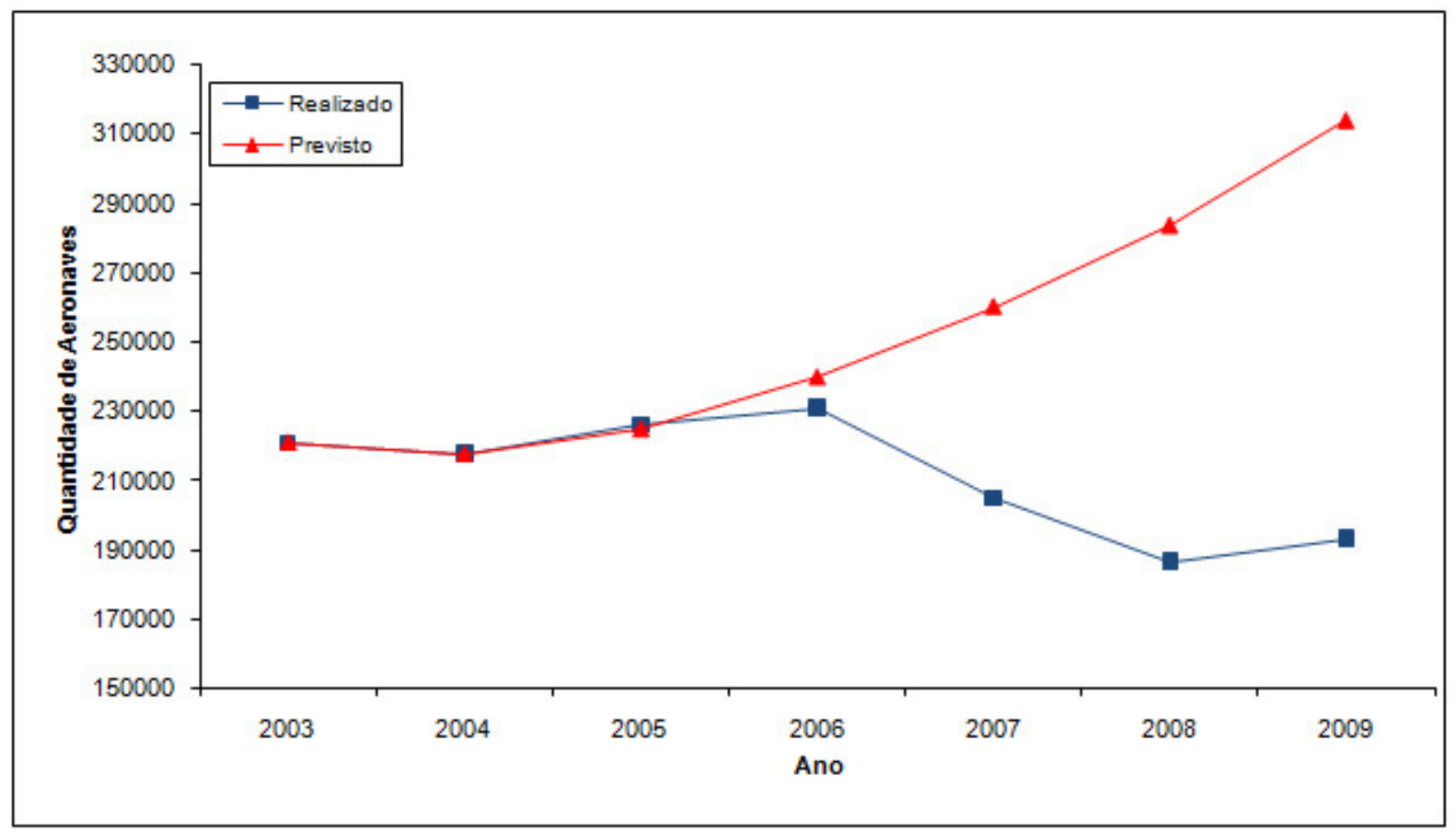

Figura 12 - Comparativo de movimentos realizados e previstos

Fonte: (INFRAERO, 2010b)

A diferença crescente entre a demanda prevista e o número de operações efetivamente realizadas impõe aos usuários restrições de oferta de voos pelas companhias aéreas que, impedidas de aumentar suas frequências em Congonhas, são obrigadas a criar novos voos partindo de outros aeroportos, como Guarulhos e Campinas.

Por outro lado, o Aeroporto de Congonhas opera próximo ao limite de sua capacidade. Nessas condições, as medidas restritivas são a única forma de manter os atrasos dos voos dentro de valores razoáveis, sem diminuir exageradamente a qualidade dos serviços oferecidos aos usuários. Os fatores que podem limitar a quantidade de tráfego são: capacidade das pistas de pouso, capacidade das pistas de rolamento, quantidade de boxes de estacionamento disponíveis, capacidade das salas de embarque / desembarque e tráfego aéreo nas proximidades do aeroporto, entre outros.

\subsection{Características do aeroporto}

Congonhas possui duas pistas de pouso, sendo a principal (17R / 35L) de 1940m de comprimento, e a auxiliar (17L / 35R) de 1435m. A pista principal conta com ILS 
categoria I em ambas as cabeceiras, o que permite operações de pouso em condições meteorológicas de até 60 metros (200 pés) de teto e 1200 metros de visibilidade. Já a pista auxiliar conta apenas com aproximações de não precisão, baseadas em estações tipo VOR / DME ou GPS. Nesse caso, os mínimos meteorológicos para pouso são 150 metros (500 pés) de teto e 1600 metros de visibilidade. A distância entre os eixos das pistas é de 220 metros, o que não permite operações simultâneas. A figura 13 mostra a planta do aeroporto.

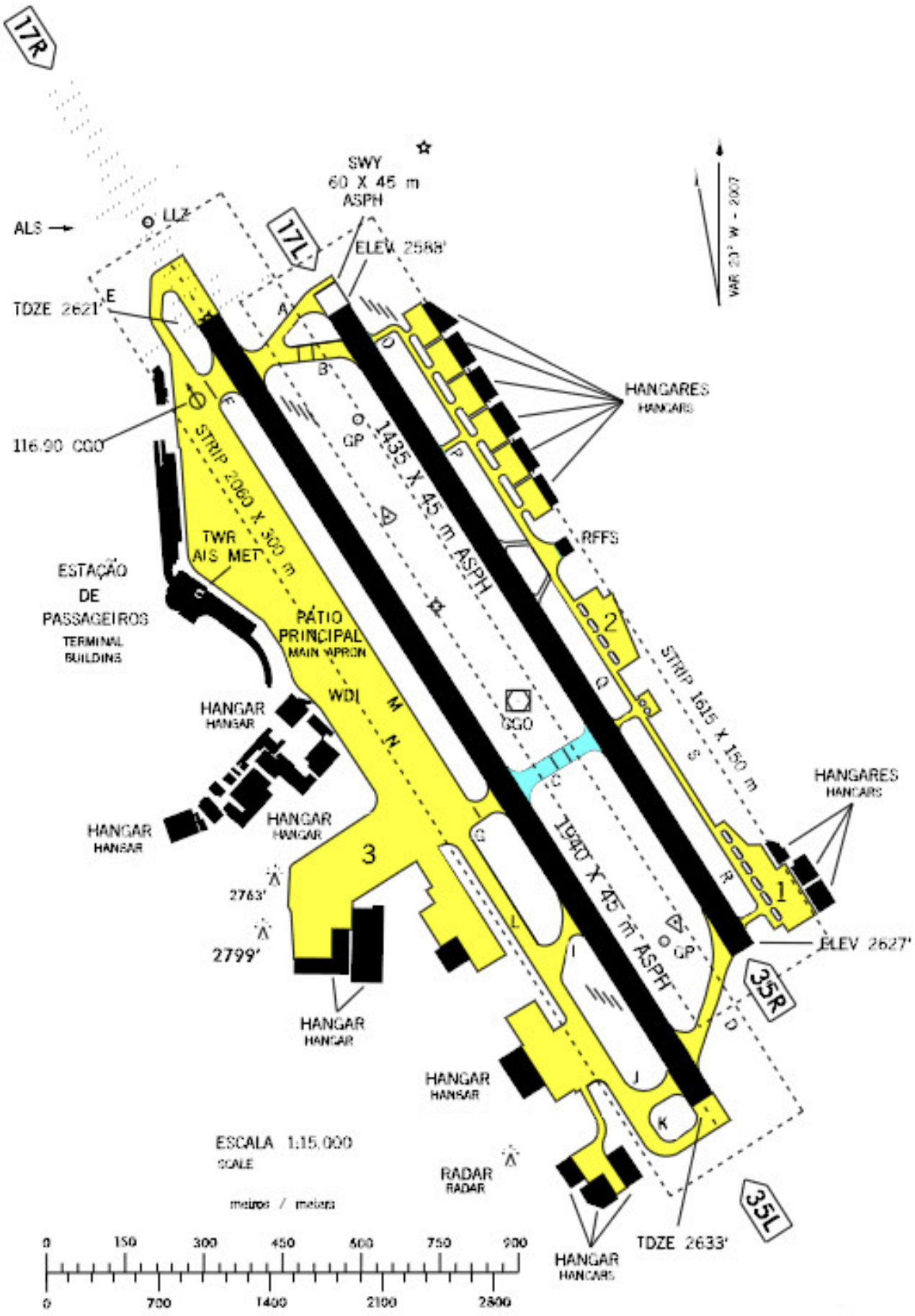

Figura 13 - Planta do Aeroporto de Congonhas Fonte: (COMANDO DA AERONÁUTICA, 2010) 
Para o estacionamento de aeronaves comerciais existem doze pontes de embarque e dezessete posições de estacionamento remotas. Devido a restrições operacionais, aeronaves tipo Fokker 100, ATR 42 e similares não podem utilizar as pontes de embarque, devendo utilizar somente as posições remotas. As aeronaves modelos Boeing 737-300 / 700 / 800 e Airbus A319 / A320 podem utilizar todas as posições de estacionamento, sem restrições. Para estacionamento de aeronaves de pequeno porte, existem dois pátios denominados pátios de aviação geral, situados do lado leste do aeroporto, além de diversos hangares particulares e de empresas de táxi aéreo. A figura 14 mostra o pátio de estacionamento para aeronaves comerciais, com as posições de estacionamento numeradas de 01 a 29.

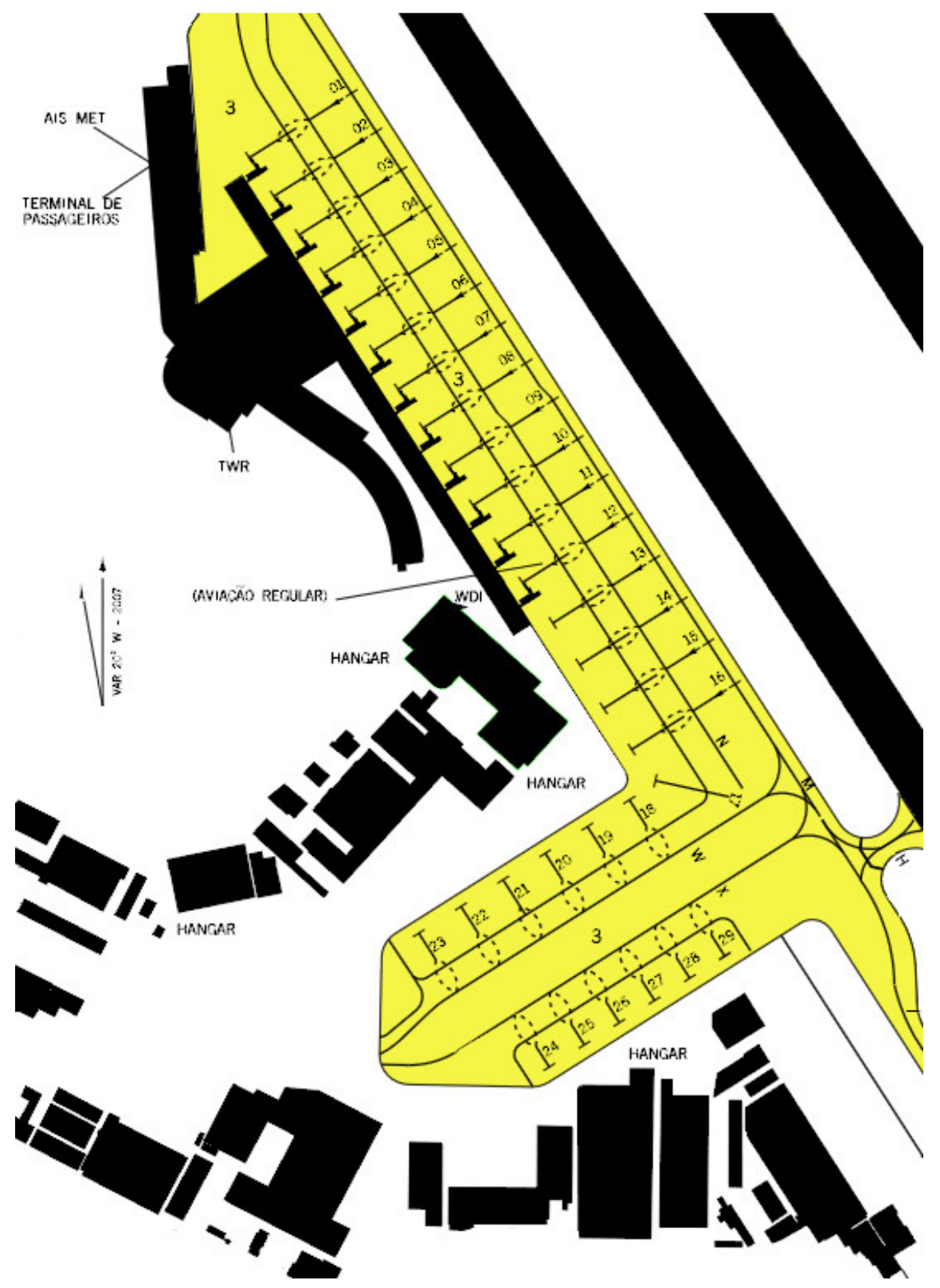

Figura 14 - Pátio de Aeronaves Comerciais Fonte: (COMANDO DA AERONÁUTICA, 2010) 
Atualmente, diversas restrições são aplicadas à operação do aeroporto, o que, de alguma forma, contribui para a diminuição da sua capacidade. As restrições impostas são:

- O aeroporto é considerado coordenado pelo CGNA do Comando da Aeronáutica; assim, somente podem operar em Congonhas voos que receberam previamente um slot para decolagem ou pouso designado por esse órgão (ANAC, 2006).

- Horário de funcionamento: não são permitidos voos entre 23:00h e 06:00h (ANAC, 2008a).

- Voos charter são permitidos apenas aos sábados, entre 14:00h e 22:45h e aos domingos, entre 06:00h e 14:00h, ainda assim, sujeitos à disponibilidade de slots (ANAC, 2008a).

- Aeronaves comerciais, quando transportando passageiros, não podem operar na pista auxiliar (ANAC, 2008b).

- Apesar das pistas principal e auxiliar possuírem 1940m e 1435m, respectivamente, as aeronaves devem operar com pesos ajustados para $1790 \mathrm{~m}$ para decolagem e $1646 \mathrm{~m}$ para pouso na pista principal, e $1345 \mathrm{~m}$ para decolagem e $1195 \mathrm{~m}$ para pouso na pista auxiliar (COMANDO DA AERONÁUTICA, 2010).

- Aeronaves não podem cruzar a pista principal quando o aeroporto estiver operando por instrumentos e existir uma aeronave que já tenha passado o marcador externo na aproximação, pois os cruzamentos interferem nos sinais de rádiofrequência do sistema ILS.

\section{2 Área Terminal de São Paulo}

A TMA de São Paulo engloba os aeroportos de Congonhas, Guarulhos, Campinas, Marte e Jundiaí, entre outros. É uma área de confluência de muitas aerovias com tráfego aéreo intenso. O órgão responsável pelo controle de tráfego na terminal é o APP São Paulo, que responde ainda pelas CTRs existentes dentro da terminal. As figuras 15 e 16 mostram os perfis lateral e vertical da TMA São Paulo. 


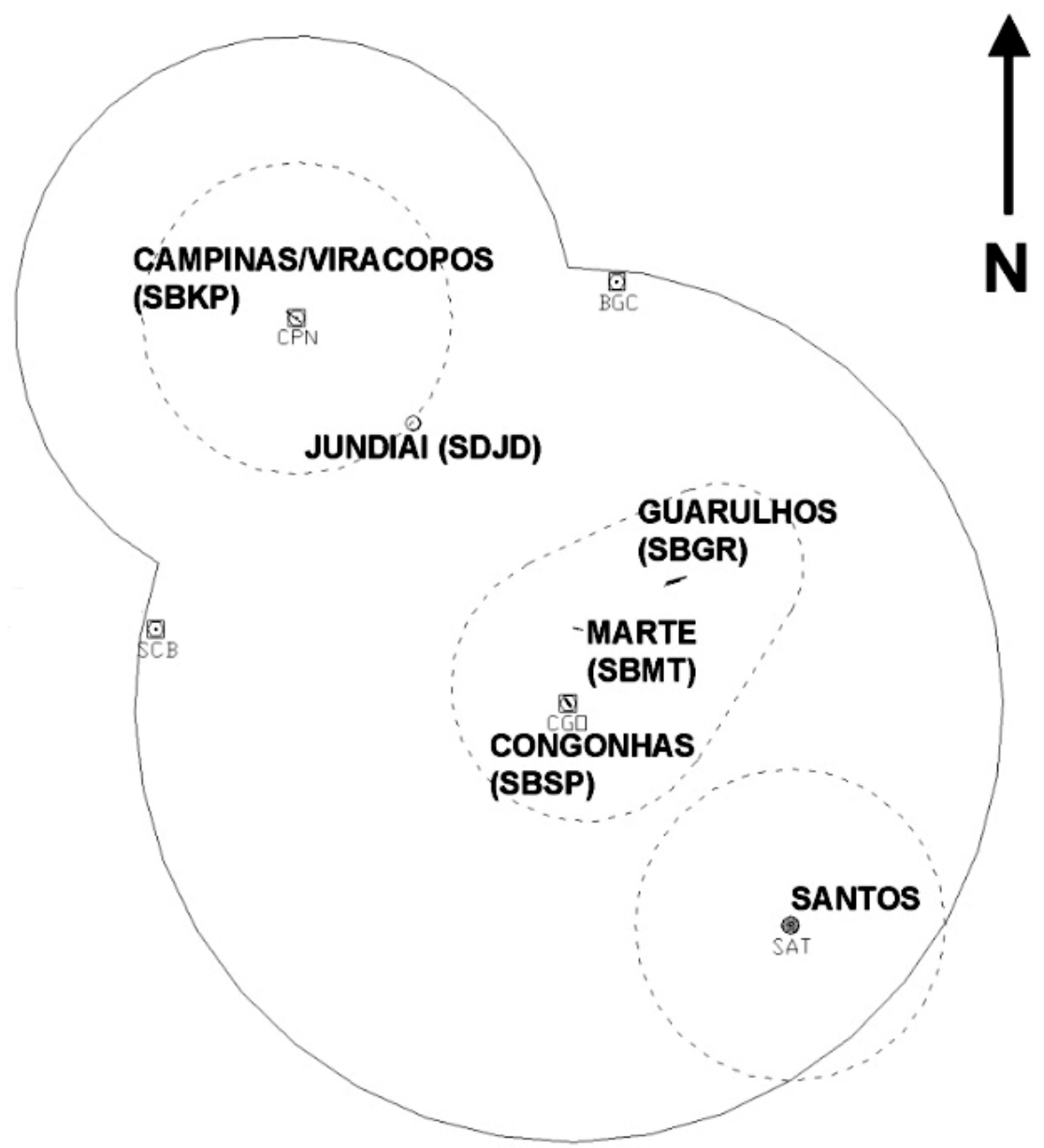

Figura 15 - Perfil lateral da TMA São Paulo Fonte: (FRAGA, 2009)

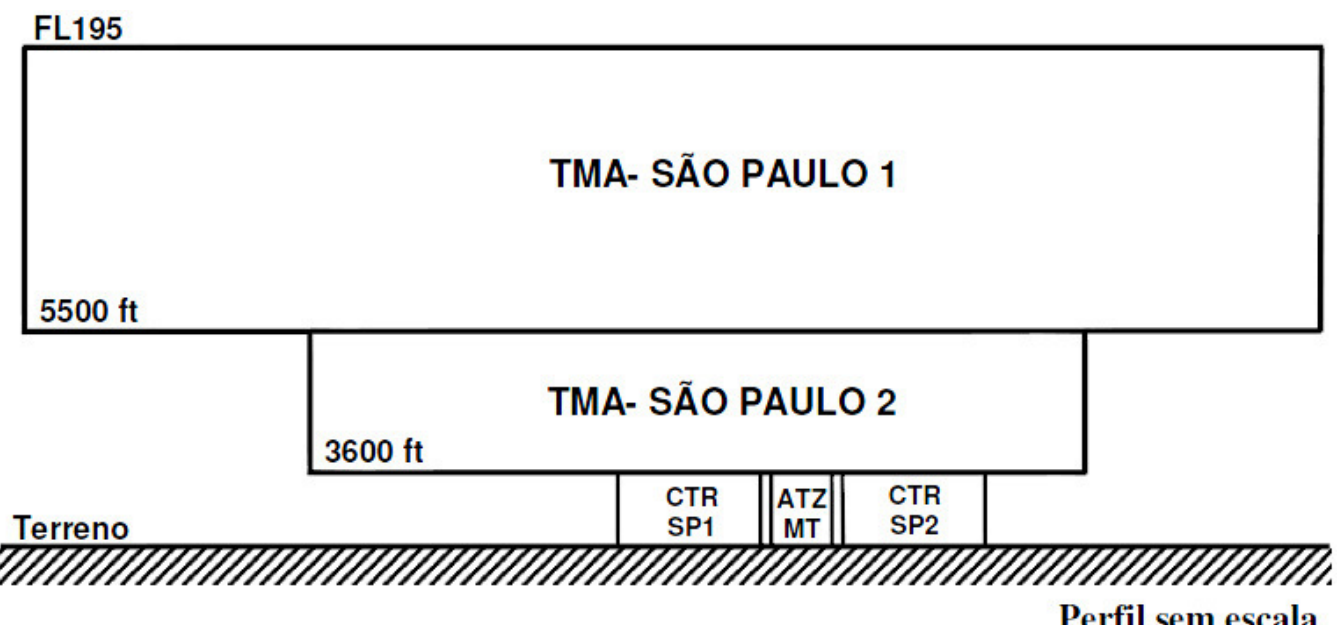

Figura 16 - Perfil vertical da TMA São Paulo Fonte: (DECEA, 2010) 


\subsubsection{Regras de tráfego aéreo específicas}

Não obstante as regras de tráfego aéreo descritas na seção 3.3, no Aeroporto de Congonhas existem regras específicas de separação que levam em consideração as condições meteorológicas reinantes (COMANDO DA AERONÁUTICA, 2009a). Os mínimos de separação aplicáveis nessas condições estão descritos na tabela 3.

Tabela 3 - Separação mínima entre aeronaves em aproximação para Congonhas Fonte: (COMANDO DA AERONÁUTICA, 2009a)

\begin{tabular}{ccc}
\hline Teto & Visibilidade & Separação mínima \\
\hline $\begin{array}{c}\text { Igual ou superior a } 1000 \\
\text { pés }(300 \text { metros })\end{array}$ & Igual ou superior a $5 \mathrm{~km}$ & $3 \mathrm{NM}$ \\
$\begin{array}{c}\text { Entre } 600 \text { e } 1000 \text { pés } \\
(180 \text { a } 300 \text { metros })\end{array}$ & Entre 2800 e 5000 metros & $4 \mathrm{NM}$ \\
$\begin{array}{c}\text { Entre } 200 \text { e } 600 \text { pés } \\
(60 \text { a } 180 \text { metros })\end{array}$ & Entre 1200 e 2800 metros & $5 \mathrm{NM}$ \\
\hline
\end{tabular}

É importante observar que, na TMA São Paulo, aviões operando segundo regras VFR e helicópteros devem seguir corredores específicos pré-estabelecidos (REA e $\mathrm{REH}$ ), de forma a não interferir com os tráfegos operando IFR (DECEA, 2010).

\subsection{Modelo construído}

No modelo criado para este estudo foram definidas as pistas de pouso, as pistas de rolamento e as posições de estacionamento para aeronaves comerciais. Para o estacionamento de aeronaves privadas, foram definidos os pátios de aviação geral 1 e 2, no lado leste do aeroporto, e dois pátios adicionais em posições onde existem hangares com grande movimento de aeronaves. A figura 17 mostra a representação do aeroporto utilizada no modelo do sítio aeroportuário construído. Para os tempos de parada de cada tipo de aeronave nas posições de estacionamento, foram utilizados os valores recomendados pelos fabricantes, por meio dos gráficos mostrados no anexo B.

De acordo com Perrella et al (2000), os ventos predominantes sopram entre sudoeste e sudeste, ocasionando maior utilização das pistas 17R e 17L. Sendo assim, para o espaço aéreo da Área Terminal de São Paulo, foram modeladas todas 
as SID e STAR para as pistas $17 \mathrm{~L}$ e $17 \mathrm{R}$, apresentadas no Anexo A. A figura 18 mostra as rotas modeladas.

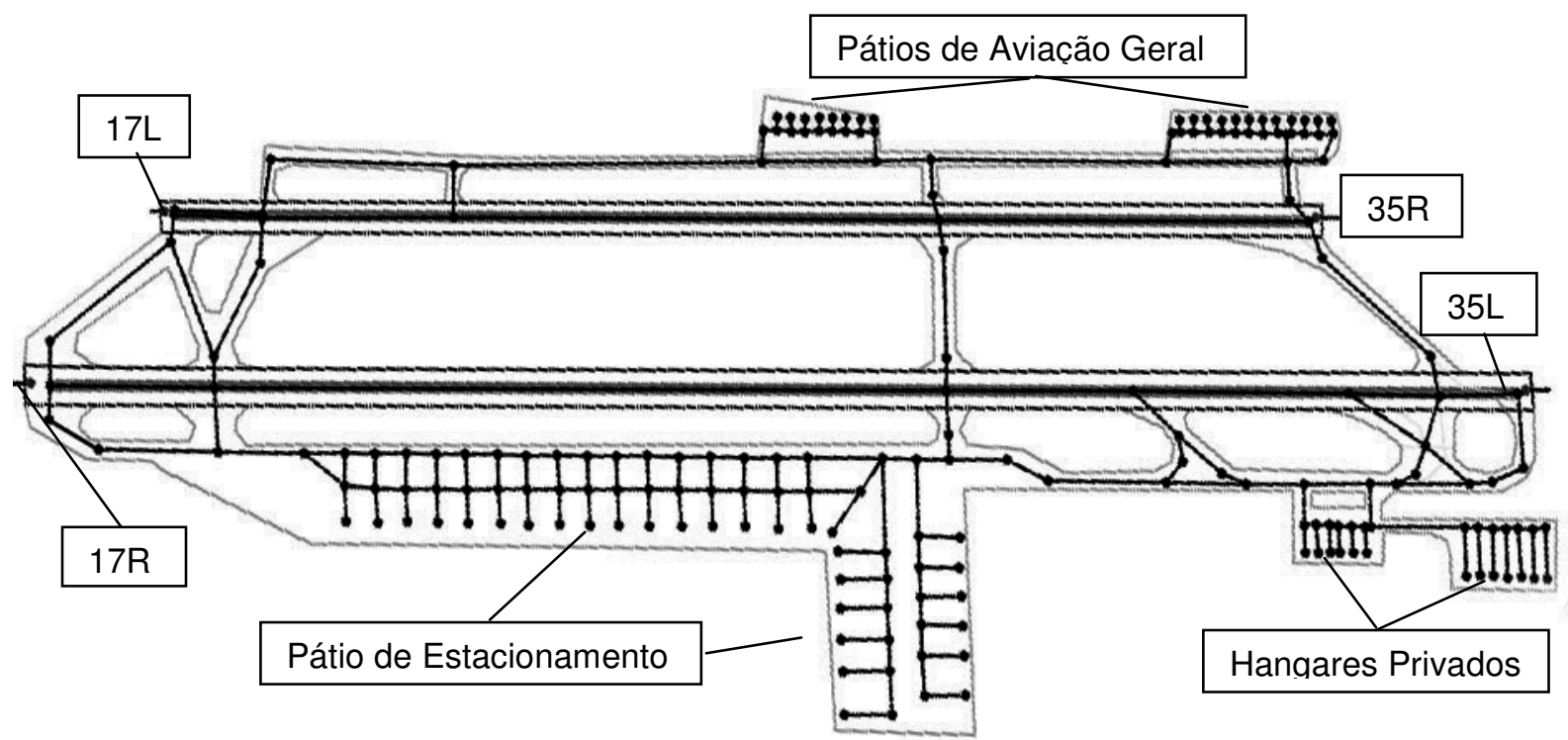

Figura 17 - Representação do Aeroporto de Congonhas no Visual SIMMOD

Foram inseridas, ainda, as regras de tráfego aéreo brasileiras, descritas na seção 3.3. Não obstante os mínimos de separação descritos nas regras, em entrevistas realizadas com controladores de tráfego aéreo da torre de controle do Aeroporto de Congonhas verificou-se que a separação de saída empregada depende de vários fatores, tais como: experiência do controlador, procedimentos de saída que serão usados, desempenho das aeronaves e contingência para falhas de comunicação, entre outros. Dessa forma, para o modelo desenvolvido neste trabalho foi proposta uma separação de noventa segundos entre decolagens, considerada adequada pelos controladores e condizente com o atualmente praticado.

Os controladores informaram também que uma instrução de trabalho específica determina que uma aeronave só poderá ser autorizada a ingressar na pista para decolagem caso uma aeronave em aproximação esteja a 7NM, pelo menos, da cabeceira da pista. Tal restrição tem por objetivo evitar aproximações perdidas causadas pela eventual demora em iniciar a corrida de decolagem, após a autorização de ingresso na pista. Essa separação mínima também foi considerada no modelo. 


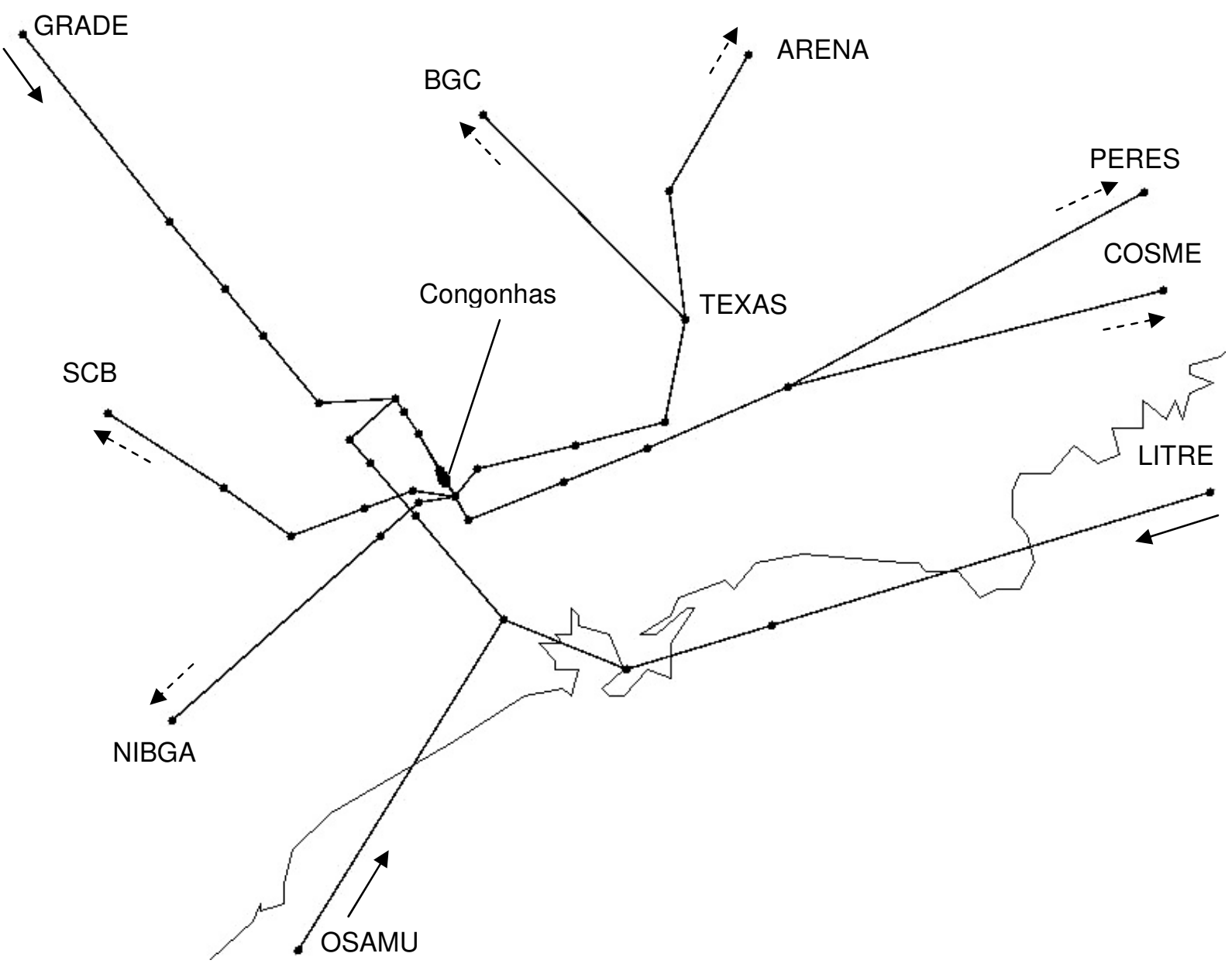

Figura 18 - Rotas de saída (SID) e chegadas (STAR) modeladas no SIMMOD

\subsection{Dados de demanda}

Uma vez que Congonhas é um aeroporto coordenado pelo CGNA, todos os voos devem receber previamente um slot para operação. A tabela do anexo C.2 apresenta os slots alocados para voos comerciais e sua análise mostra que não há dia pico de operação. De fato, os mesmos voos são realizados diariamente, de segunda a sexta-feira, havendo apenas uma diminuição aos sábados e domingos. Sendo assim, foram inseridos no software todos os voos regulares que possuem slots designados pelo CGNA para dias úteis. A tabela 4 apresenta a quantidade de voos alocados por hora de operação do aeroporto. Analisando a tabela, é possível notar pequenas variações entre o total de movimentos nas 17 horas de operação do aeroporto, e também variações no balanceamento entre decolagens e pousos alocados para cada hora, conforme mostrado na figura 19. 
Além dos slots para voos comerciais, o CGNA aloca quatro slots por hora, sendo um a cada 15 minutos, para a aviação geral, que opera aeronaves de menor porte.

Tabela 4 - Alocação de slots para voos comerciais Fonte: (CGNA, 2009)

\begin{tabular}{cccc}
\hline Horários & Decolagens & Pousos & Total \\
\hline 06:00-06:59 & 17 & 11 & 28 \\
07:00-07:59 & 12 & 17 & 29 \\
08:00-08:59 & 19 & 12 & 31 \\
09:00-09:59 & 11 & 19 & 30 \\
10:00-10:59 & 18 & 13 & 31 \\
11:00-11:59 & 14 & 16 & 30 \\
12:00-12:59 & 14 & 15 & 29 \\
13:00-13:59 & 17 & 13 & 30 \\
14:00-14:59 & 11 & 21 & 32 \\
15:00-15:59 & 17 & 10 & 27 \\
16:00-16:59 & 12 & 18 & 30 \\
17:00-17:59 & 16 & 15 & 31 \\
18:00-18:59 & 16 & 14 & 30 \\
19:00-19:59 & 15 & 16 & 31 \\
20:00-20:59 & 14 & 17 & 31 \\
21:00-21:59 & 14 & 18 & 32 \\
22:00-22:59 & 11 & 6 & 17 \\
Total & 248 & 251 & 499 \\
\hline
\end{tabular}

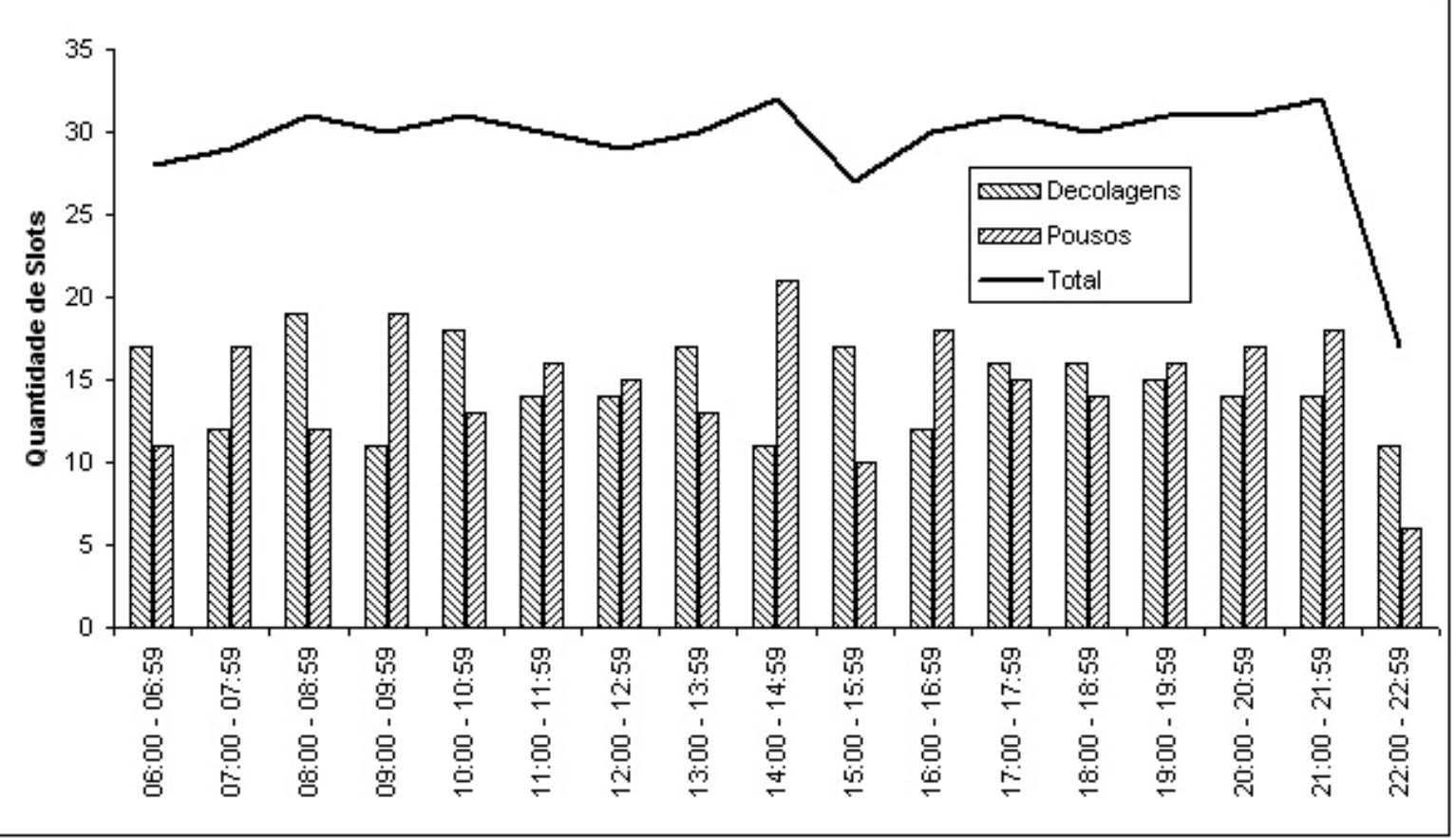

Figura 19 - Movimentos por hora em Congonhas

Fonte: (CGNA, 2009) 


\subsection{Validação do modelo de simulação}

Após a inserção dos voos, inicialmente foi feita uma análise visual do funcionamento do modelo com a ferramenta Animator, a fim de identificar possíveis erros em procedimentos, conflitos e gridlocks. Ao final desse passo, o modelo encontrou-se com todas as rotas, tanto aéreas como de táxi, corretas e sem conflitos. A figura 20 mostra um exemplo de análise com o Animator.

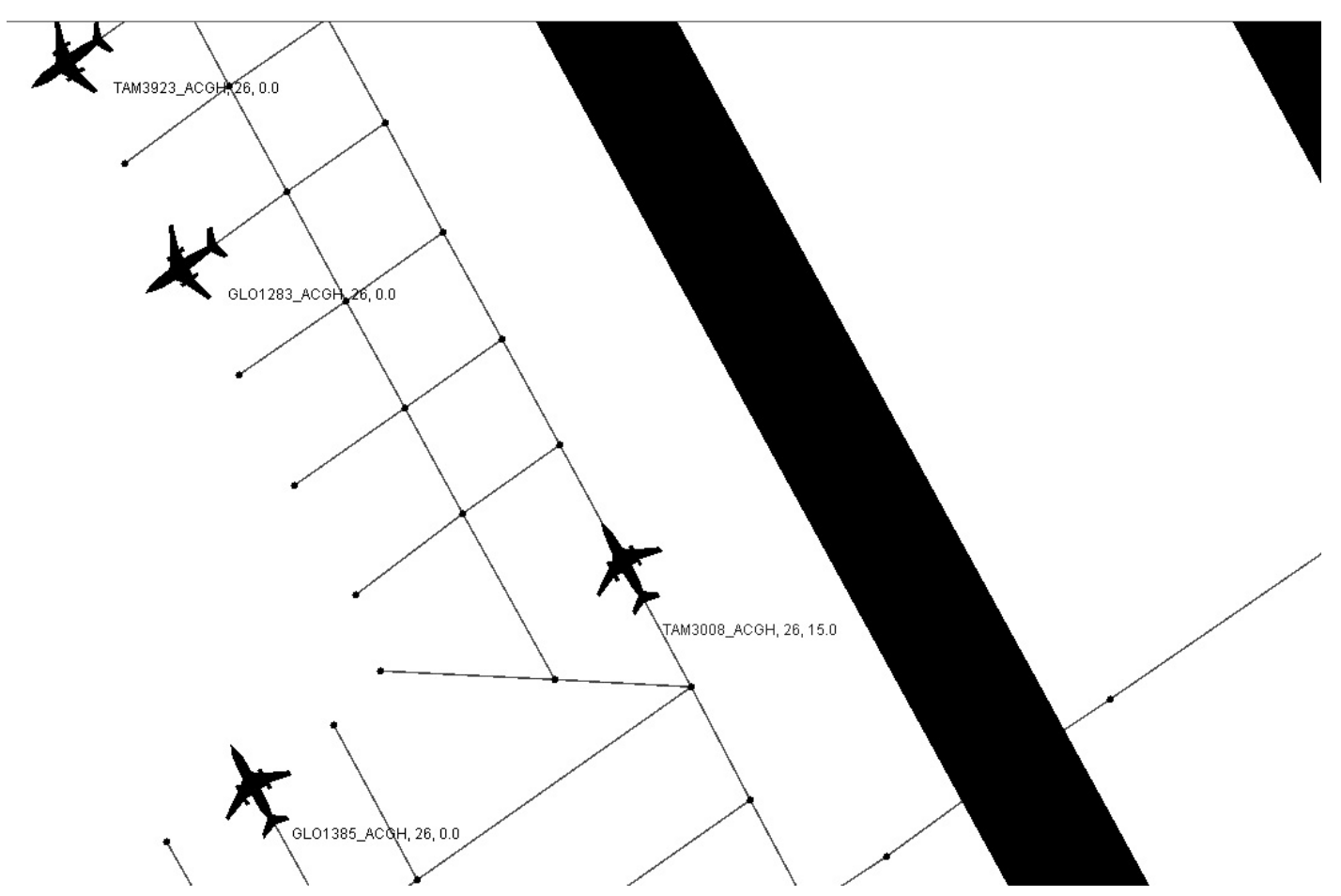

Figura 20 - Verificação de rotas de solo com a ferramenta Animator

Em seguida, passou-se à análise dos dados quantitativos, comparando-se o total de voos simulados e sua distribuição pelas rotas de saída e chegada com a tabela do Anexo C.2. O objetivo dessa fase é verificar se todos os voos foram criados com origem e destino corretos. A tabela 5 mostra a quantidade de voos simulada por rota de saída e chegada. Por tratar-se de uma quantidade de movimentos relativamente pequena e distribuída de forma diferente a cada dia, os slots da aviação geral não foram incluídos no modelo durante a fase de validação, mas estão presentes em todos os outros cenários simulados.

A última parte da validação consistiu em verificar o funcionamento do modelo ao longo do tempo, comparando a quantidade de decolagens e pousos ocorridos em 
cada hora da simulação com os dados da tabela 4. Nessa fase, foram validadas as regras de separação inseridas no modelo, analisando-se as possíveis ondas de atraso e sua propagação em relação ao previsto.

Tabela 5 - Quantidade de voos simulados por rota

\begin{tabular}{|c|c|c|c|c|}
\hline Tipo & Rota & Transição & Quantidade & Total \\
\hline \multirow{6}{*}{ Saída } & APIT & NIBGA & 61 & \multirow{6}{*}{248} \\
\hline & \multirow{2}{*}{ DIADEMA } & PERES & 23 & \\
\hline & & COSME & 69 & \\
\hline & \multirow{2}{*}{ DUMO } & TEXAS & 29 & \\
\hline & & $\mathrm{BGC}$ & 40 & \\
\hline & $\mathrm{KING}$ & SCB & 26 & \\
\hline \multirow{5}{*}{ Chegada } & \multirow{2}{*}{ CELSO 17} & GRADE & 70 & \multirow{5}{*}{251} \\
\hline & & PCL & 28 & \\
\hline & \multirow{3}{*}{ REDE 17} & PNG & 15 & \\
\hline & & OSAMU & 43 & \\
\hline & & LITRE & 95 & \\
\hline \multicolumn{4}{|c|}{ Total } & 499 \\
\hline
\end{tabular}

Os cálculos de validação são detalhados no apêndice B. Ao final do processo de análise e ajuste foi obtido o gráfico da figura 21, que mostra as diferenças entre movimentos previstos e simulados por hora.

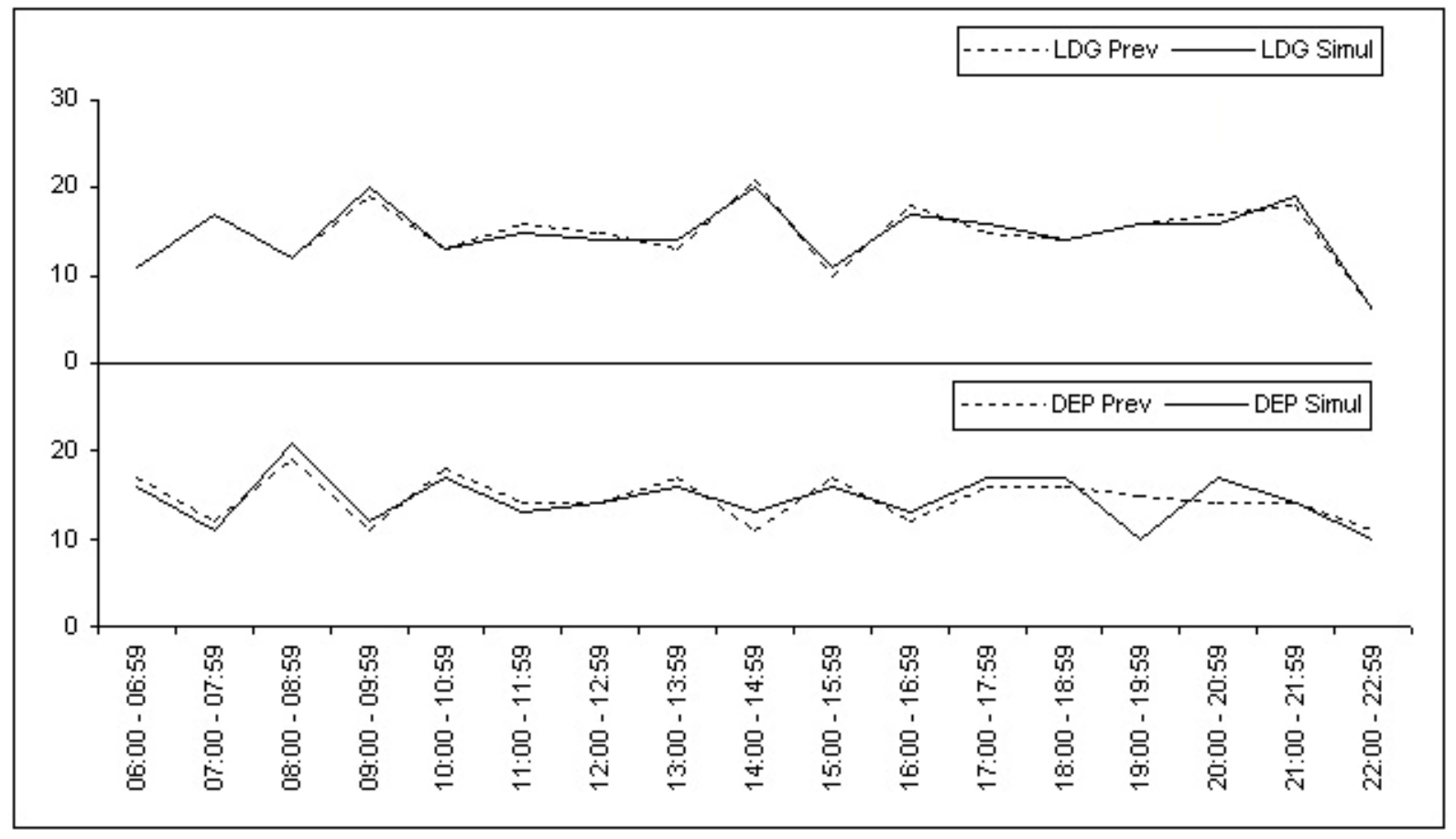

Figura 21 - Comparação entre movimentos previstos e simulados 
Observando o gráfico, é possível notar que os pousos (LDG) sofrem variações menores que as decolagens (DEP). Essa diferença corresponde à realidade, pois, de acordo com as regras de tráfego aéreo, as aeronaves pousando têm prioridade sobre as que vão decolar (COMANDO DA AERONÁUTICA, 2009b). Outra característica do modelo é diluir os voos que não puderam operar na hora prevista, na hora subsequente. Isto também corresponde ao que ocorre na realidade, já que o espaçamento entre as aproximações é aumentado quando existe acúmulo de tráfego para decolagem, para permitir um fluxo maior de decolagens.

\subsection{Cenários alternativos}

Tendo sido concluída a fase de validação, o modelo encontrou-se pronto para a simulação dos cenários alternativos de operação do aeroporto. Foram, então, criados diversos cenários a fim de verificar o comportamento do sistema sob variações de demanda e com alterações de procedimentos.

Para a definição dos cenários que seriam simulados, foram calculadas capacidades teóricas com o FAA Airport Capacity Model e esses dados foram confrontados com a distribuição diária de slots. A figura 22 apresenta os envelopes de capacidade para 0 cenário com separação de 7NM entre aproximações e 90 segundos entre decolagens (operação atual) e um outro, com separações de 5NM / 60 segundos. Os envelopes mostram que o volume de operações excede a capacidade no cenário atual e que as reduções de separação têm potencial para aumento da capacidade do aeroporto. Com isso, foram definidos os seguintes cenários para serem simulados:

- Separação de 90 segundos entre decolagens e 7NM entre aproximações;

- Separação de 60 segundos entre decolagens e 7NM entre aproximações;

- Separação de 90 segundos entre decolagens e 5NM entre aproximações;

- Separação de 60 segundos entre decolagens e 5NM entre aproximações;

- Substituição de aeronaves turbo-hélices por jatos;

- Reorganização da tabela de alocação de slots. 


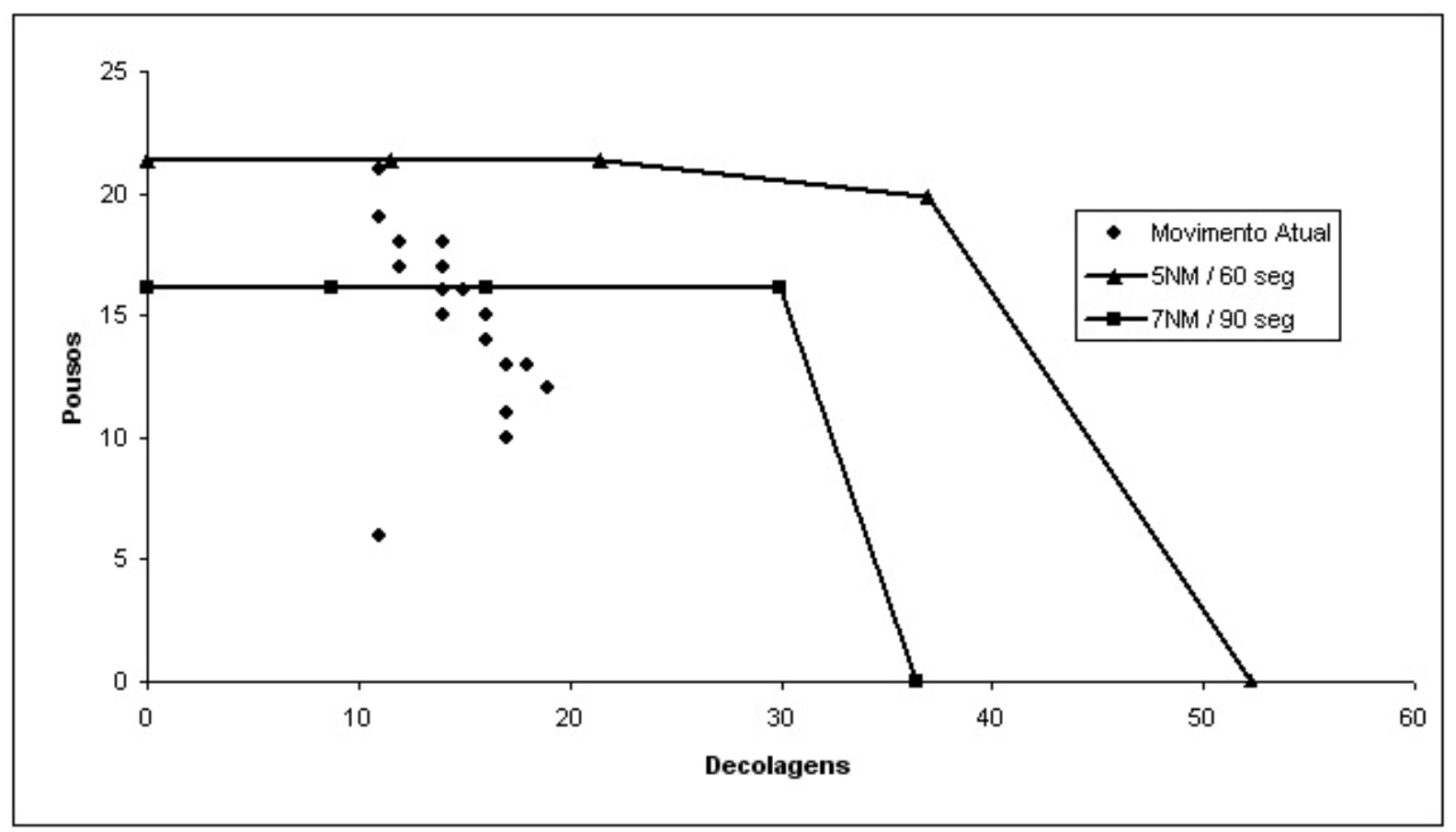

Figura 22 - Distribuição atual de slots e envelopes de capacidade de Congonhas

\subsubsection{Cenário atual}

No modo atual de operação, os controladores de tráfego aéreo empregam separação de 90 segundos entre decolagens consecutivas e 7NM de separação entre aeronaves em aproximação, a fim de permitir uma decolagem intercalada entre duas aproximações. A tabela 6 apresenta os resultados obtidos com a separação atual e as variações de demanda propostas em 4.5.1. Note-se que o aeroporto encontra-se saturado, sendo necessária uma redução de tráfego da ordem de 9.9\% para que o atraso médio chegue a um nível aceitável.

Tabela 6 - Atraso médio - cenário simulado: 7NM / 90 seg

\begin{tabular}{ccc}
\hline Demanda & $\begin{array}{c}\text { Número de voos } \\
\text { diários }\end{array}$ & $\begin{array}{c}\text { Atraso médio } \\
\text { para decolagem } \\
\text { (minutos) }\end{array}$ \\
\hline Redução de 15\% & 492 & 3.07 \\
Redução de 10\% & 517 & 5.07 \\
Redução de 5\% & 542 & 7.51 \\
Atual & 567 & 9.52 \\
Aumento de 5\% & 593 & 12.42 \\
Aumento de 10\% & 617 & 15.28 \\
Aumento de 15\% & 642 & 23.49 \\
\hline
\end{tabular}




\subsubsection{Separação de 60 segundos entre decolagens}

Considerando a recomendação da ICAO de uma separação mínima de 60 segundos entre decolagens consecutivas, foi criado outro cenário, combinando separações de 60 segundos entre decolagens e 7NM entre aproximações, com as variações de demanda propostas. A tabela 7 apresenta do resultados obtidos nessas condições. Neste cenário, note-se que 0 aeroporto opera em condição seriamente congestionado, sendo necessária uma redução de $9 \%$ no volume de tráfego para que o atraso médio seja considerado aceitável.

\begin{tabular}{cc} 
Tabela 7 - Atraso médio - cenário simulado: $7 \mathrm{NM} / 60 \mathrm{seg}$ \\
\hline Demanda & $\begin{array}{c}\text { Atraso médio } \\
\text { para decolagem } \\
\text { (minutos) }\end{array}$ \\
\hline Redução de 15\% & 3.03 \\
Redução de 10\% & 5.21 \\
Redução de 5\% & 5.86 \\
Atual & 7.58 \\
Aumento de 5\% & 11.98 \\
Aumento de 10\% & 13.95 \\
Aumento de 15\% & 22.57
\end{tabular}

\subsubsection{Separação de 5NM entre aproximações}

De acordo com as regras apresentadas em 3.3.1 e as regras específicas de operação do Aeroporto de Congonhas (5.2.1), a separação entre aeronaves em aproximação poderia ser reduzida para até 3NM quando operando sob boas condições meteorológicas. No entanto, além de não ser possível operar com essa separação sob condições adversas, também não é possível intercalar uma decolagem entre duas aproximações separadas em 3NM.

Já uma separação de $5 \mathrm{NM}$ pode ser utilizada mesmo em condições meteorológicas de operação por instrumentos e permite uma decolagem entre duas aproximações consecutivas. Por isso, optou-se por criar um cenário de simulação combinando a separação de $5 \mathrm{NM}$ com as variações de demanda consideradas anteriormente. É importante destacar que a separação entre decolagens foi novamente ajustada para 
90 segundos. O objetivo desse retorno é avaliar o impacto de cada modificação separadamente. A tabela 8 apresenta os resultados dessa simulação. Note-se, neste cenário, que o aeroporto encontra-se congestionado, sendo necessária uma redução do volume de tráfego da ordem de $7 \%$ no volume de tráfego para que 0 atraso médio torne-se aceitável.

Tabela 8 - Atraso médio - cenário simulado: 5NM / 90 seg

\begin{tabular}{cc}
\hline Demanda & $\begin{array}{c}\text { Atraso médio } \\
\text { para decolagem } \\
\text { (minutos) }\end{array}$ \\
\hline Redução de 15\% & 2.79 \\
Redução de 10\% & 4.71 \\
Redução de 5\% & 5.53 \\
Atual & 6.49 \\
Aumento de 5\% & 10.94 \\
Aumento de 10\% & 14.36 \\
Aumento de 15\% & 19.47 \\
\hline
\end{tabular}

\subsubsection{Combinação dos cenários de 60 segundos e 5NM}

Visando a verificar a capacidade do sistema num cenário otimizado e dentro das normas recomendadas, foi criado um cenário de simulação combinando-se os anteriores, ou seja, separação entre decolagens de 60 segundos e $5 \mathrm{NM}$ entre aproximações. A tabela 9 apresenta os resultados desse cenário com a aplicação das demandas estipuladas. Note-se que com a combinação de separações utilizada, o aeroporto é considerado congestionado com o volume de tráfego atual e uma redução da ordem de 3.5\% é necessária para que os atraso médio fique aceitável.

\subsubsection{Substituição de aeronaves turbo-hélices por jatos}

As aeronaves com propulsão por motores turbo-hélices têm desempenho inferior às aeronaves a jato e, com isso, utilizam velocidades menores durante a aproximação, podendo causar uma diminuição do fluxo de aeronaves e consequente aumento dos atrasos. Para verificar o impacto da operação dessas aeronaves, foi criado um cenário onde as aeronaves ATR 42 foram substituídas por Airbus A320. O cenário com essa substituição foi combinado com as separações de 7NM / 90 segundos e 
5NM / 60 segundos, além das variações de demanda. A tabela 10 mostra os resultados obtidos. Note-se que no cenário com 7NM e 90 segundos de separação, o aeroporto apresenta-se seriamente congestionado e uma redução de cerca de $8 \%$ no volume de tráfego é necessária para que o atraso médio fique aceitável. Já no cenário que utiliza separações de $5 \mathrm{NM}$ e 60 segundos, o atraso médio é considerado aceitável com o volume de tráfego atual.

Tabela 9 - Atraso médio - cenário simulado: 5NM / 60 seg

\begin{tabular}{cc}
\hline Demanda & $\begin{array}{c}\text { Atraso médio } \\
\text { para decolagem } \\
\text { (minutos) }\end{array}$ \\
\hline Redução de 15\% & 2.12 \\
Redução de 10\% & 3.93 \\
Redução de 5\% & 4.36 \\
Atual & 5.65 \\
Aumento de 5\% & 8.61 \\
Aumento de 10\% & 10.97 \\
Aumento de 15\% & 18.35 \\
\hline
\end{tabular}

Tabela 10 - Atraso médio - cenário simulado: aeronaves a jato

\begin{tabular}{ccc}
\hline Demanda & $\begin{array}{c}\text { Atraso médio para decolagem (minutos) } \\
\text { Separação } \\
\text { 7NM / 90 seg }\end{array}$ & $\begin{array}{c}\text { Separação } \\
\text { 5NM / 60 seg }\end{array}$ \\
\hline Redução de 15\% & 3.21 & 2.34 \\
Redução de 10\% & 4.48 & 3.35 \\
Redução de 5\% & 6.46 & 3.74 \\
Atual & 8.19 & 4.91 \\
Aumento de 5\% & 12.33 & 8.21 \\
Aumento de 10\% & 14.68 & 11.23 \\
Aumento de 15\% & 24.58 & 22.57 \\
\hline
\end{tabular}

\subsubsection{Reorganização da tabela de alocação de slots}

Observando a tabela de alocação de slots do CGNA, apresentada no anexo C.2, verifica-se que alguns slots sequenciais são alocados a voos que decolam para o mesmo destino ou, em outros casos, usando a mesma subida. A tabela 11 mostra um exemplo dessas ocorrências. 
Tabela 11 - Exemplo de decolagens sucessivas usando a mesma SID

\begin{tabular}{|c|c|c|c|c|c|c|c|}
\hline Slot ATC & Voo & Origem & Destino & Eqpto & Tipo & Procedimento & Transição \\
\hline $6: 17$ & TAM3900 & & SBRJ & A319 & DEP & DIADEMA & COSME \\
\hline $6: 19$ & GLO1500 & & SBRJ & B737 & DEP & DIADEMA & COSME \\
\hline $6: 21$ & GLO1346 & & SBVT & B737 & DEP & DIADEMA & PERES \\
\hline
\end{tabular}

Essa sucessão de voos seguindo a mesma trajetória pode levar a atrasos para decolagem e ainda a restrições impostas às aeronaves em voo, durante a subida, para que a separação mínima seja mantida. Para verificar se é possível reduzir os níveis de atraso modificando a tabela de slots, foi criado um cenário com trocas de slots entre voos, de forma a evitar ao máximo decolagens sucessivas na mesma rota. Os voos modificados encontram-se no anexo C.6. A tabela 12 mostra os resultados da simulação do cenário, combinado com as separações de 7NM / 90 seg e com as variações de demanda. Note-se que os atrasos com a modificação da tabela de slots são maiores que na condição atual de operação, com o aeroporto permanecendo saturado para a demanda atual. Uma redução da ordem de $16 \%$ é necessária para que o nível de serviço oferecido às aeronaves passe a ser adequado.

Tabela 12 - Atraso médio - cenário simulado: reorganização de slots

\begin{tabular}{cc}
\hline Demanda & $\begin{array}{c}\text { Atraso médio } \\
\text { para decolagem } \\
\text { (minutos) }\end{array}$ \\
\hline Redução de 15\% & 5.42 \\
Redução de 10\% & 6.57 \\
Redução de 5\% & 7.28 \\
Atual & 11.22 \\
Aumento de 5\% & 16.22 \\
Aumento de 10\% & 29.88 \\
Aumento de 15\% & 109.57 \\
\hline
\end{tabular}

\subsection{Conclusões do capítulo}

Nesse capítulo foram apresentadas as principais características do Aeroporto de Congonhas e da Área Terminal de São Paulo, bem como descritas a criação e validação de um modelo de simulação para o Aeroporto, de acordo com a metodologia proposta. A partir do modelo inicial validado, foram considerados 
diversos cenários de operação e apresentados os resultados obtidos em cada um, segundo as métricas propostas. No próximo capítulo é feita uma análise comparativa desses resultados, assim como comparações entre cenários diferentes. 


\section{ANÁLISE COMPARATIVA DOS RESULTADOS}

Em uma primeira análise da simulação do cenário atual de operação (separações de $7 \mathrm{NM}$ e 90 segundos), pode-se obter o gráfico da figura 23, a partir do dados apresentados na tabela 6 .

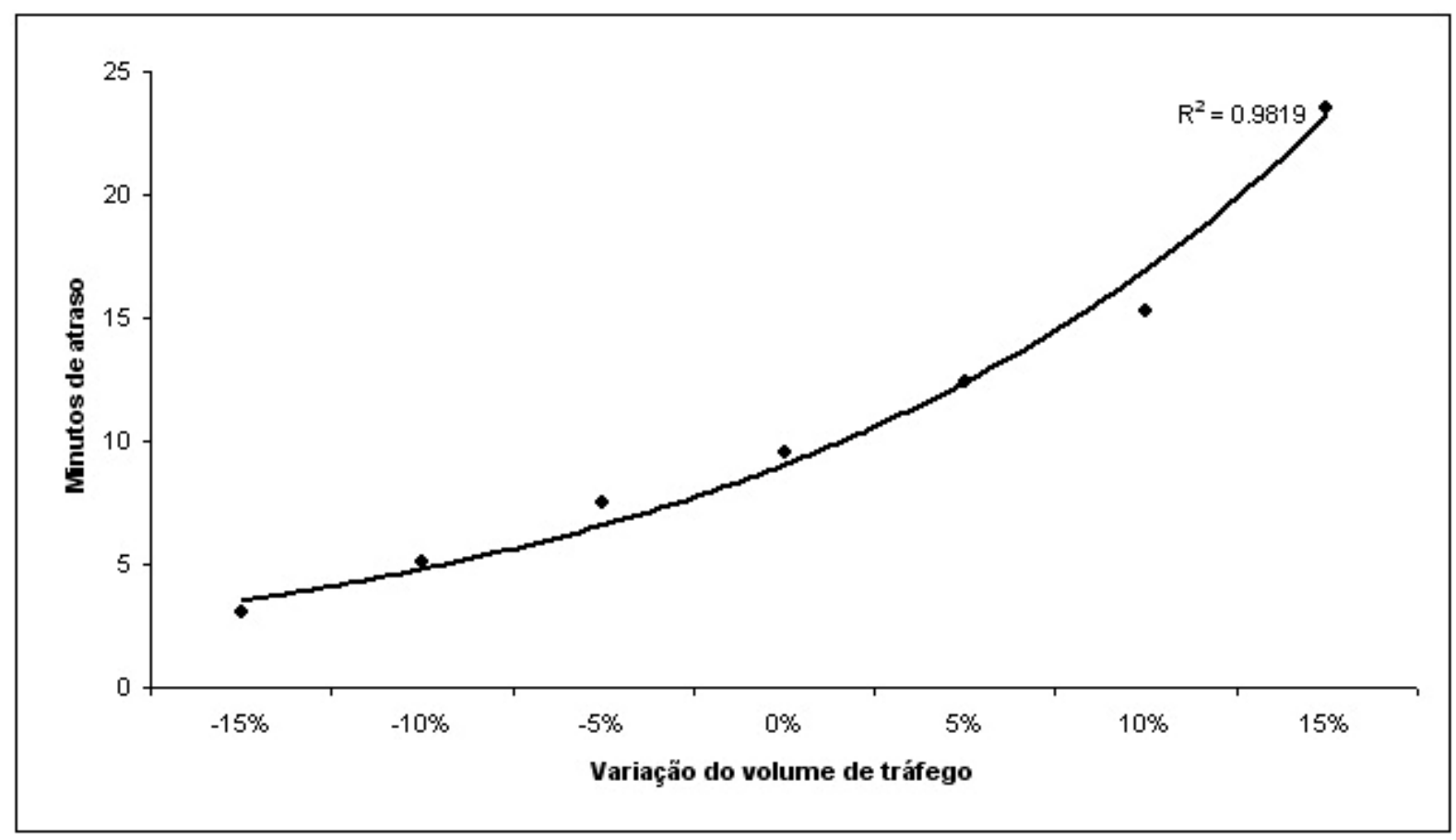

Figura 23 - Atraso médio em função do volume de tráfego - separação de 7NM / 90 segundos

O gráfico mostra que os atrasos obtidos com os sete níveis de volume de tráfego são consistentes com o encontrado na literatura, que afirma que os atrasos aumentam de forma exponencial quando o aeroporto está operando próximo ao limite de capacidade (DE NEUFVILLE, 1976 e HORONJEFF e MCKELVEY, 1994). Essa consistência assegura a confiabilidade do modelo para uso nos próximos cenários. Também é possível inferir que o Aeroporto de Congonhas encontra-se saturado, de acordo com a escala de nível de serviço adotada, mostrada na tabela 1.

Como descrito na metodologia (seção 4.3), o atraso para decolagem é calculado por meio da soma do atraso na posição de estacionamento, do tempo de atraso no táxi e do tempo de espera na fila de decolagem. Decompondo-se essa soma, foi obtido o 
gráfico da figura 24, que mostra a variação de cada uma dessas parcelas de atraso em função do volume de tráfego.

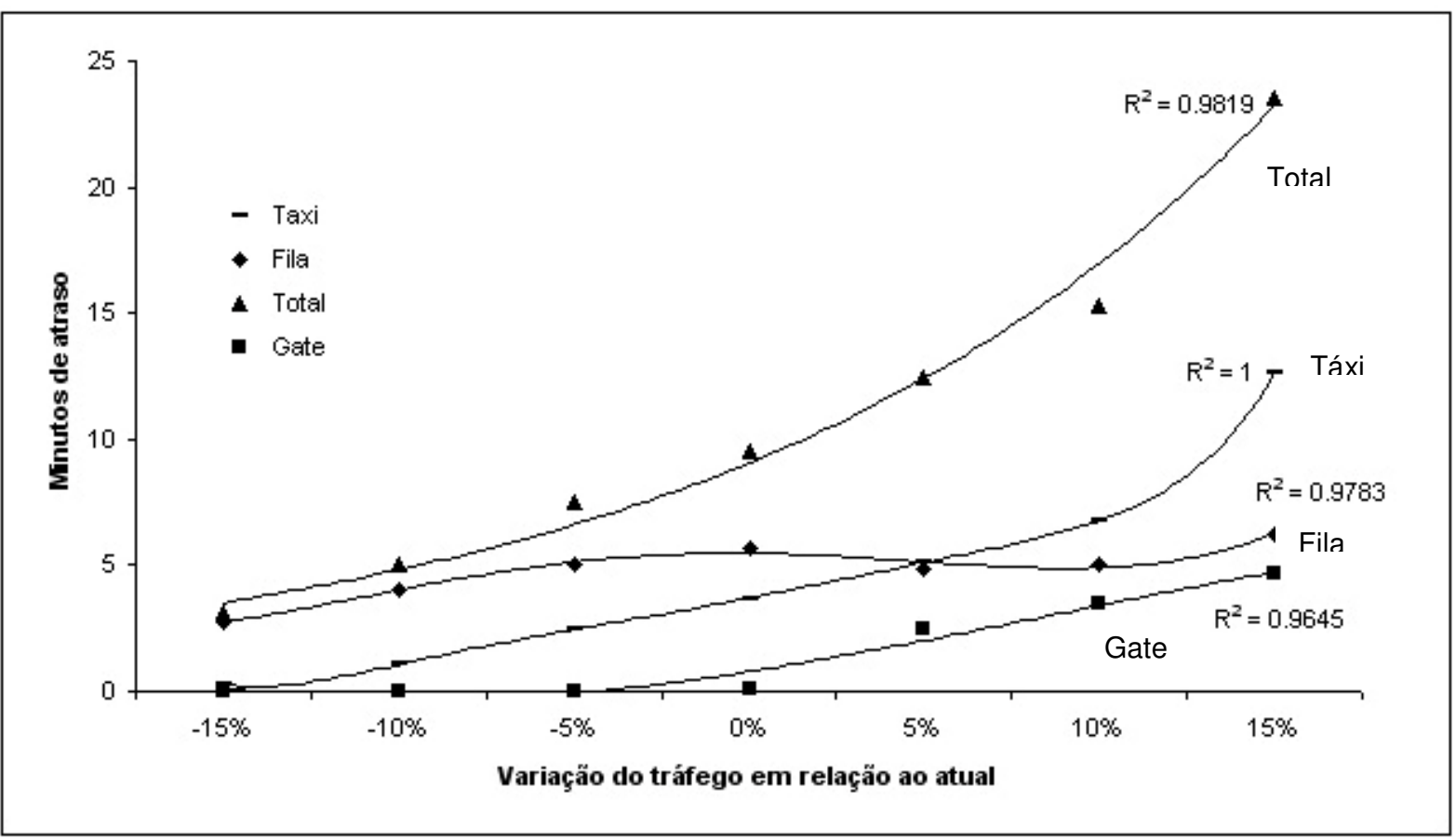

Figura 24 - Decomposição do atraso médio para decolagem - separação 7NM / 90 segundos

O gráfico mostra que, com baixas demandas, a fila de decolagem ainda não opera na capacidade máxima, o que passa a ocorrer com demanda entre $-5 \%$ e a atual. $\mathrm{A}$ partir desse ponto, o tempo de atraso na fila é praticamente constante, pois, como a fila tem um tamanho definido, as aeronaves esperam o mesmo tempo para percorrer toda a fila. Com isso, passam a existir atrasos na posição de estacionamento, já que as aeronaves aguardam estacionadas até que exista uma posição livre na fila de decolagem.

Já o atraso durante o táxi aumenta de forma praticamente linear com a demanda, até cerca de $10 \%$ acima do volume atual e, acima disso, o aumento torna-se exponencial. $\mathrm{O}$ aumento de atraso no táxi deve-se principalmente às pistas de táxi "X" e "W", que comportam, cada uma, apenas uma aeronave, visto que possuem apenas uma entrada / saída. Com o aumento do volume de tráfego, aumenta também a quantidade de aeronaves que utilizam as posições 17 a 23, servidas pela pista de táxi "W", e 24 a 29, servidas pela pista de táxi "X", levando a maiores esperas no táxi. 
Outra análise que pode ser feita é do tempo de ocupação das posições de estacionamento das aeronaves comerciais. O gráfico da figura 25 mostra a taxa de ocupação dos gates em função do volume de tráfego. A taxa de ocupação para cada nível de demanda é calculada por meio da razão entre a soma dos tempos de permanência das aeronaves nos gates pelo tempo total disponível (17 horas de operação do aeroporto multiplicado por 29 gates).

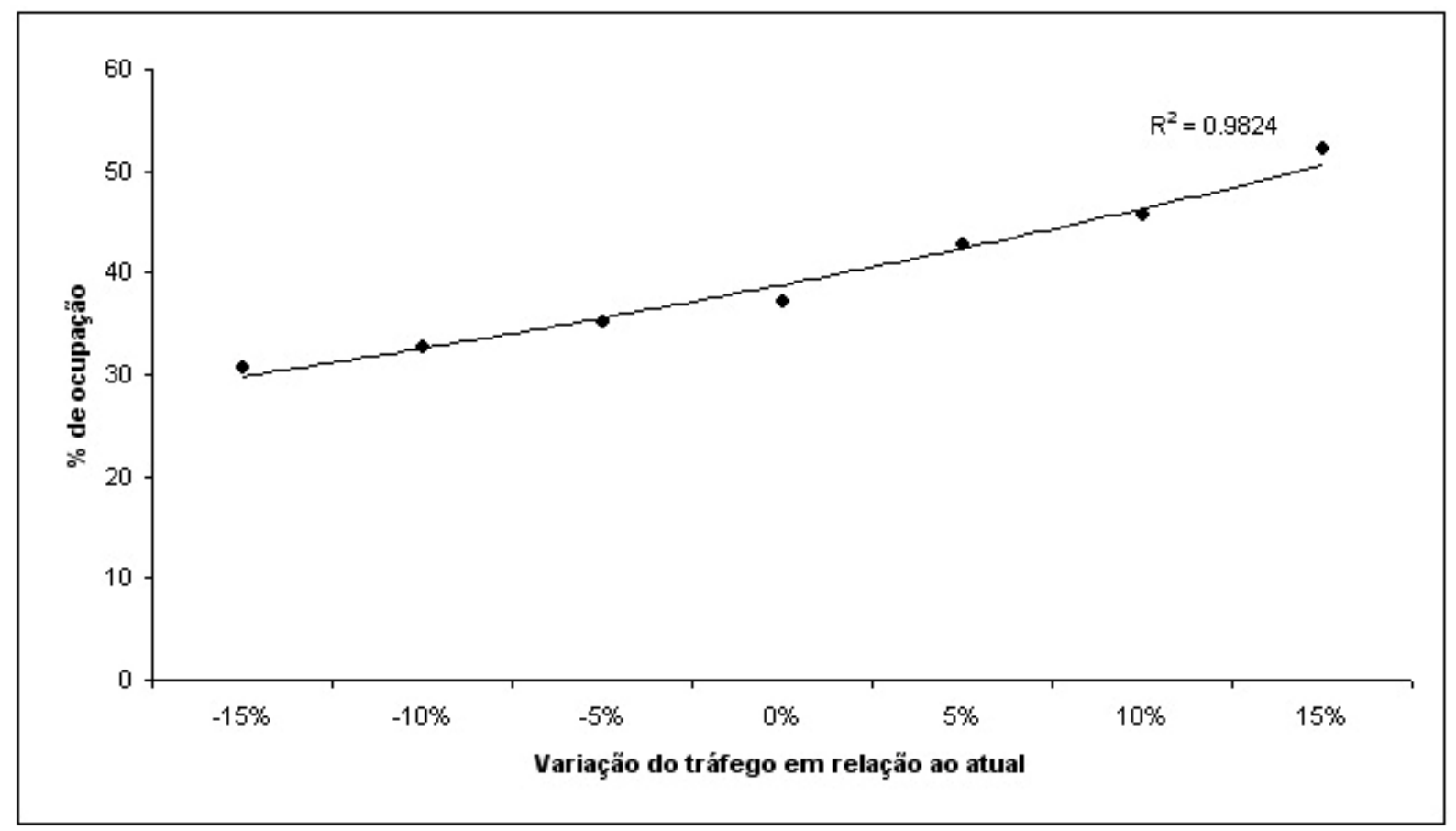

Figura 25 - Taxa de ocupação das posições de estacionamento - Cenário 7NM / 90seg

O gráfico indica que a ocupação das posições de estacionamento aumenta de forma consistente com o aumento de demanda e que esse subsistema tem capacidade para absorver aumento de demanda, já que a taxa de ocupação média é de pouco mais de $50 \%$, mesmo considerando uma demanda $15 \%$ superior à atual.

É importante notar que a análise de ocupação das posições de estacionamento foi realizada com base nos tempos ótimos de trânsito (turn around time) informados pelos fabricantes e apresentados no Anexo B. No entanto, na operação diária, as empresas frequentemente deixam as aeronaves estacionadas por tempos maiores, aguardando a conexão entre voos. Não foi possível simular esses tempos, pois isso depende de dados restritos às empresas que variam diariamente em função dos 
trilhos alocados à cada aeronave. Com isso, é possível que a ocupação real dos gates seja superior aos resultados aqui apresentados.

\subsection{Comparação entre os cenários}

Com os resultados dos cenários de simulação, apresentados nas tabelas 6 a 12, foram construídos a tabela 13 e o gráfico da figura 26. A comparação entre os cenários mostra que a redução de separação é eficaz para redução de atrasos e melhora do nível de serviço do aeroporto. A redução de 90 para 60 segundos entre decolagens permite uma redução de $20,4 \%$ nos atrasos para a demanda atual. A redução de separação de $7 \mathrm{NM}$ para $5 \mathrm{NM}$, diminui os atrasos em $31,8 \%$. Já a combinação das reduções de separação entre decolagens e aproximações permite uma redução de atrasos da ordem de $40,6 \%$ para a demanda atual.

Tabela 13: Comparação entre os cenários simulados

\begin{tabular}{ccccccccc}
\hline Cenário & $\begin{array}{c}\text { Número } \\
\text { de voos } \\
\text { diários }\end{array}$ & $\begin{array}{c}\text { Cenário } \\
\text { atual }\end{array}$ & $\begin{array}{c}60 \mathrm{seg} / \\
7 \mathrm{NM}\end{array}$ & $\begin{array}{c}90 \mathrm{seg} / \\
5 \mathrm{NM}\end{array}$ & $\begin{array}{c}60 \mathrm{seg} / \\
5 \mathrm{NM}\end{array}$ & $\begin{array}{c}\text { Subst. de turbo- } \\
\text { hélices por jatos } \\
90 \mathrm{seg} / \\
7 \mathrm{NM}\end{array}$ & $\begin{array}{c}\text { Reorg. } \\
\text { tabela } \\
\text { te slots } \\
5 \mathrm{NM}\end{array}$ & $\begin{array}{c}\text { 90seg/ } \\
7 N M\end{array}$ \\
\hline Redução de 15\% & 492 & 3.07 & 3.03 & 2.79 & 2.12 & 3.21 & 2.34 & 5.42 \\
Redução de 10\% & 517 & 5.07 & 5.21 & 4.71 & 3.93 & 4.48 & 3.35 & 6.57 \\
Redução de 5\% & 542 & 7.51 & 5.86 & 5.53 & 4.36 & 6.46 & 3.74 & 7.28 \\
Atual & 567 & 9.52 & 7.58 & 6.49 & 5.65 & 8.19 & 4.91 & 11.22 \\
Aumento de 5\% & 593 & 12.42 & 11.98 & 10.94 & 8.61 & 12.33 & 8.21 & 16.22 \\
Aumento de 10\% & 617 & 15.28 & 13.95 & 14.36 & 10.97 & 14.68 & 11.23 & 29.88 \\
Aumento de 15\% & 642 & 23.49 & 22.57 & 19.47 & 18.35 & 24.58 & 22.57 & 109.57 \\
\hline
\end{tabular}

\subsection{Substituição de aeronaves turbo-hélices por jatos}

Comparando-se os resultados das simulações do cenário atual com um cenário onde apenas aeronaves a jato são operadas (tabelas 6, 9 e 10), foi construído o gráfico da figura 27. Esse gráfico mostra que a substituição de aeronaves reduz os atrasos em cerca de 13\%, tanto no cenário com separação de $7 \mathrm{NM} / 90$ segundos quanto com 5NM / 60 segundos. Com isso, no cenário com 7NM / 90 segundos, o aeroporto passa de saturado para seriamente congestionado e, no cenário com 5NM 
/ 60 segundos, passa de congestionado para aceitável, de acordo com a escala de nível de serviço adotada.

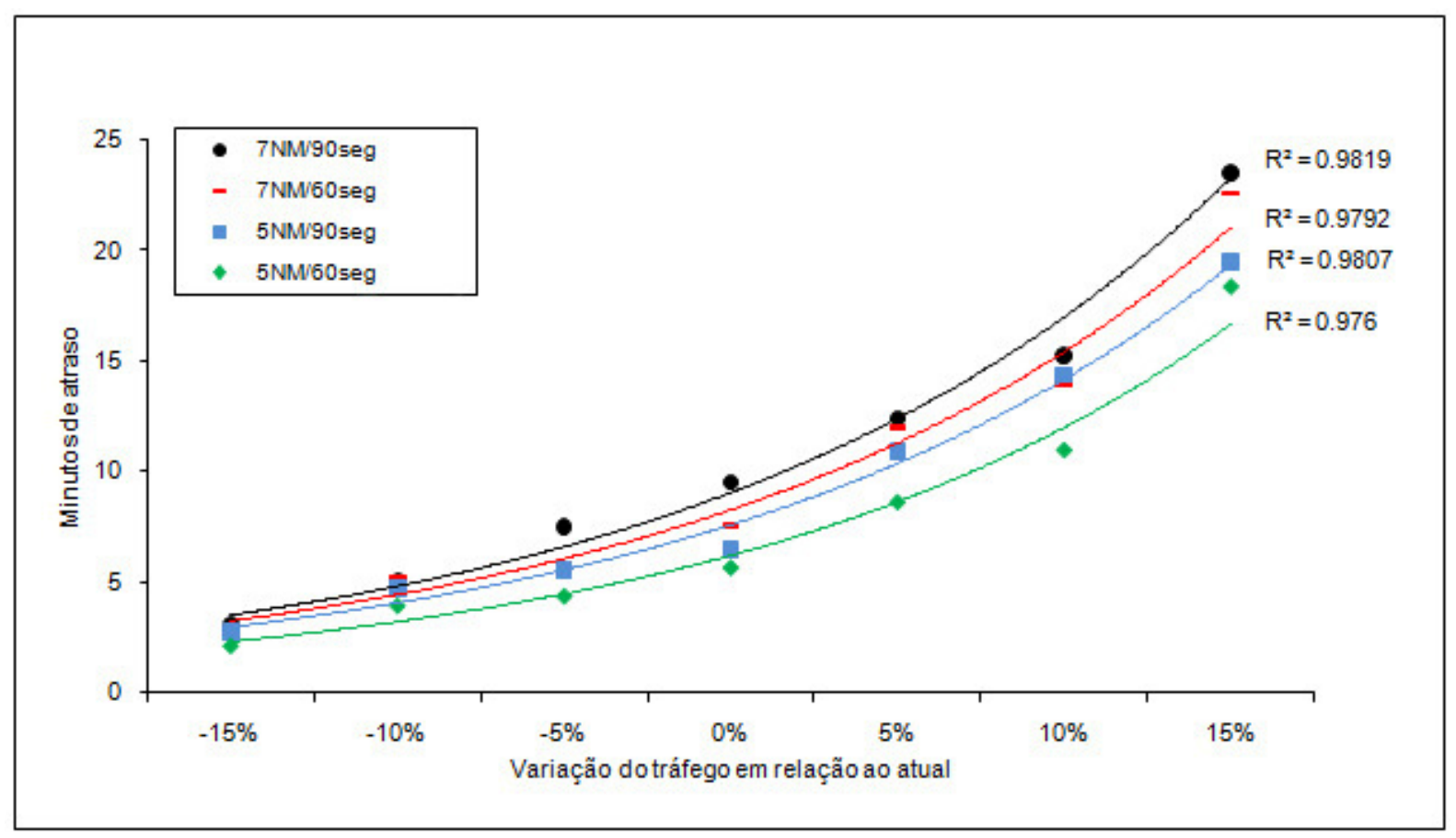

Figura 26 - Comparação entre os cenários simulados

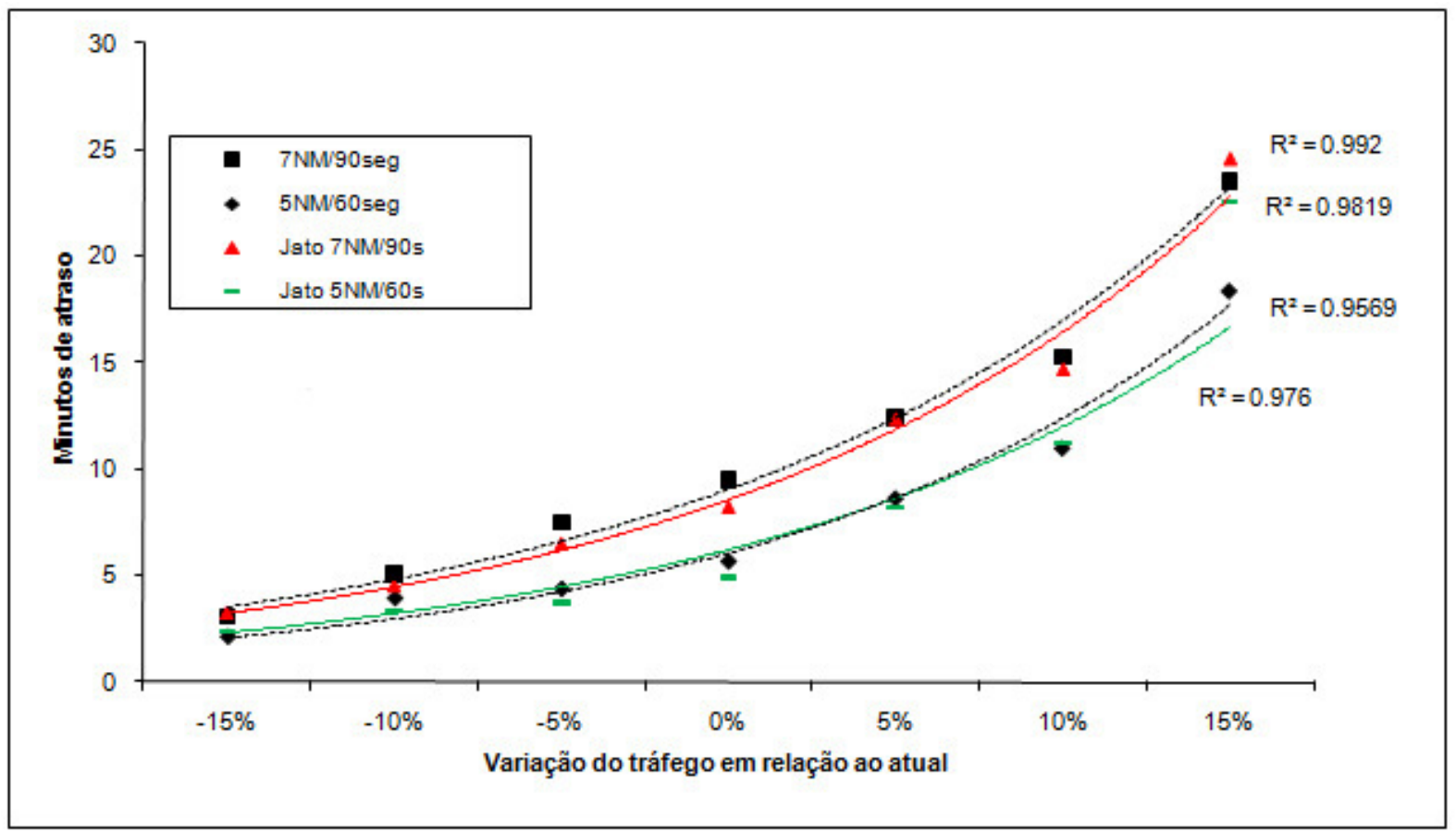

Figura 27 - Comparação entre os cenários com substituição de turbo-hélices por jatos 


\subsection{Reorganização da tabela de slots}

A figura 28 mostra a comparação entre o cenário atual e o cenário com os slots reordenados, ambos com separação de 7NM / 90 segundos.

A modificação da tabela de slots, de forma a separar as decolagens consecutivas, gerou um aumento dos atrasos médios. Tal aumento pode ser explicado pela característica de operação de aeródromos com uma única pista, onde a maior capacidade horária é atingida quando há apenas decolagens. Dessa forma, quando há, na tabela de slots, vários voos decolando sem nenhuma aproximação intercalada, o aeroporto está, ainda que momentaneamente, operando em sua capacidade máxima. A figura 23, apresentada na seção 5.5, mostra os envelopes de capacidade do Aeroporto de Congonhas, onde essa característica de capacidade máxima de decolagens pode ser observada.

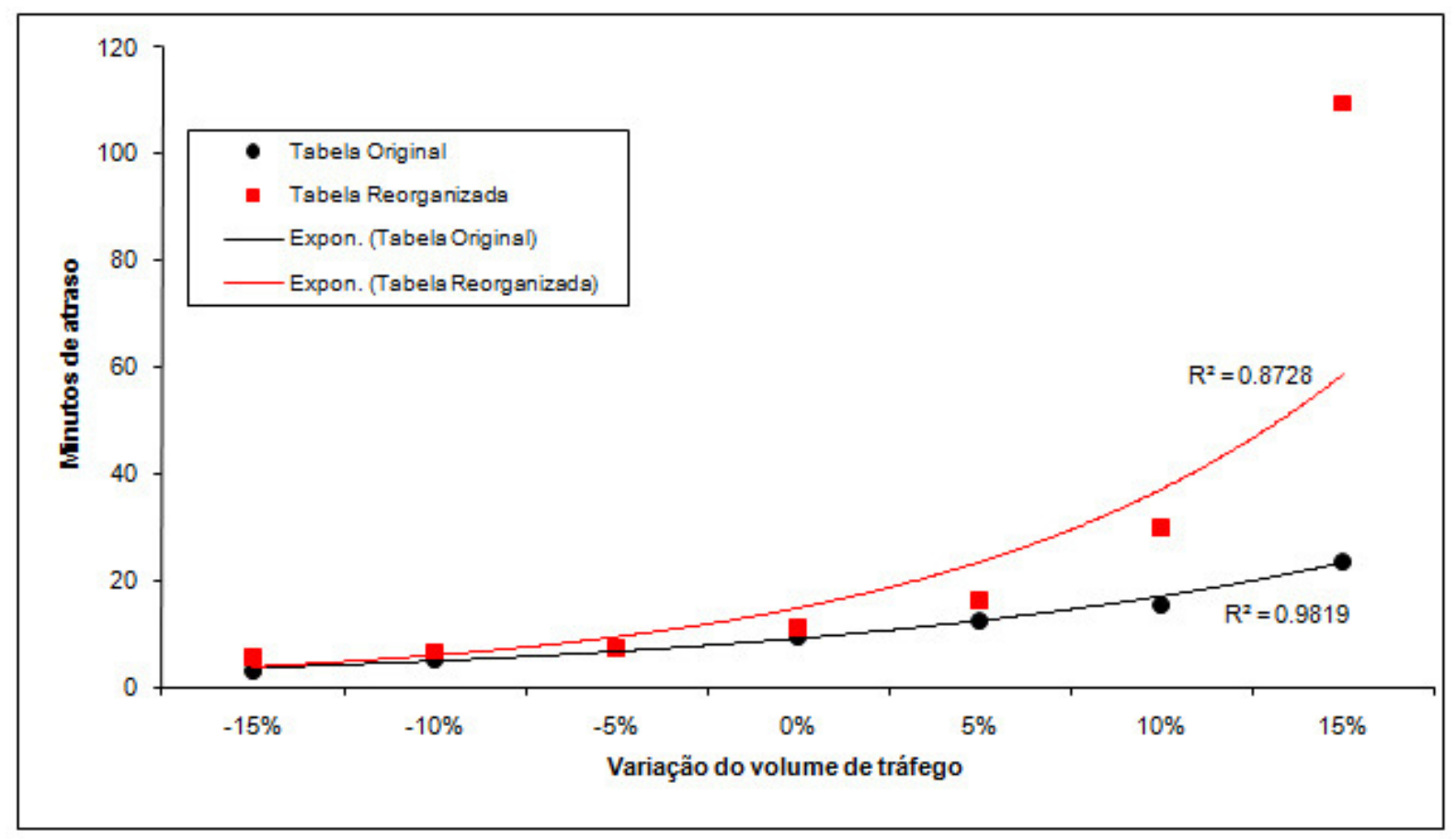

Figura 28 - Comparação entre os cenários com reorganização de slots

\subsection{Conclusões do Capítulo}

Nesse capítulo foi apresentada uma análise dos diversos cenários simulados para o Aeroporto de Congonhas, bem como uma comparação entre eles. A comparação 
mostra que alguns cenários apresentam-se eficazes para a redução do atraso médio para decolagem das aeronaves, como as reduções de separação entre aeronaves na aproximação, por exemplo. Já outros cenários apresentaram-se ineficazes, como a modificação da tabela de slots. No próximo capítulo são apresentadas as conclusões finais do trabalho. 


\section{CONCLUSÕES, RECOMENDAÇÕES E TRABALHOS FUTUROS}

O objetivo deste trabalho foi apresentar uma metodologia de avaliação da capacidade do lado aéreo de aeroportos associada ao nível de serviço oferecido às aeronaves. Para tanto, foram reunidos conceitos de abordagem sistêmica, capacidade associada a nível de serviço e ferramentas de simulação, úteis para esse tipo de abordagem. A metodologia foi aplicada ao Aeroporto de Congonhas.

A metodologia descrita mostrou-se eficaz para avaliação de capacidade do lado aéreo de aeroportos, bem como para propor cenários capazes de melhorar o nível de serviço oferecido pelo aeroporto analisado.

De acordo com os resultados obtidos, o Aeroporto de Congonhas encontra-se saturado nas condições atuais de operação, levando as aeronaves a esperas excessivas no solo, com nível de serviço abaixo do aceitável. Também é possível concluir que há um desbalanceamento entre os componentes analisados: enquanto a limitação operacional é imposta pelo subsistemas pistas de pouso e de táxi, as posições de estacionamento apresentam capacidade ociosa.

A partir de uma regressão linear com dados obtidos no cenário de operação atual, conclui-se que uma redução do volume de tráfego da ordem de 9,9\% é necessária para que o aeroporto passe a operar com um nível de serviço aceitável. Isso equivale à redução de 49 voos por dia.

Os cenários alternativos mostram que é possível melhorar sensivelmente o nível de serviço oferecido às aeronaves sem necessidade de investimentos em infraestrutura, apenas com modificações nos procedimentos operacionais de controle de tráfego aéreo.

Assim, num cenário com redução da separação entre decolagens para 60 segundos e mantendo-se a separação entre aproximações em 7NM, há uma redução de quase 2 minutos no atraso médio das aeronaves, mas uma redução de tráfego da ordem de $9 \%$ ainda se faz necessária para que o atraso seja aceitável. 
Num cenário com modificação da separação entre aproximações para 5NM, mas mantendo-se o intervalo entre decolagens em 90 segundos, o aeroporto passa de saturado para congestionado e uma redução de volume de tráfego de $7 \%$ é necessária para que o atraso seja aceitável.

Num cenário que combina as modificações da separação entre aproximações de 7NM para 5NM, e entre decolagens de 90 para 60 segundos, o nível de serviço passa de saturado para congestionado. É necessária, então, uma redução de 3,7\% no volume de operações para atingir-se um nível de serviço aceitável, o que representa a redução de 19 voos por dia. A adoção de saídas por instrumentos baseadas em vetoração radar é uma alternativa viável para a efetivação das mudanças nas separações entre decolagens. Essas modificações exigem, no entanto, redimensionamento e capacitação do pessoal dos órgãos ATC envolvidos (torre e controle de aproximação), bem como a familiarização dos pilotos das empresas que operam em Congonhas com os novos procedimentos.

Um cenário que alia as modificações nas regras de tráfego aéreo com a substituição de aeronaves turbo-hélices por jatos apresenta-se como a melhor opção de operação do aeroporto, levando a que o nível de serviço seja aceitável sem necessidade de redução na quantidade de operações. No entanto, a implantação desse cenário depende de uma análise de viabilidade econômica e financeira por parte da empresa que opera as aeronaves turbo-hélices, o que pode dificultar ou mesmo inviabilizar esse cenário, em função dos investimentos envolvidos na substituição.

A reordenação da tabela de slots intercalando-se pousos e decolagens mostrou-se ineficaz, levando a um aumento do atraso médio. Nesse aspecto, pode-se concluir que a ordenação atual dos voos é mais eficaz para a operação do aeroporto.

Entre as várias possibilidades que se apresentam para ampliação e aprofundamento do trabalho, destacam-se as seguintes: 
- Estudos que determinem o nível de risco associado ao aumento da capacidade do aeroporto a partir da utilização da pista auxiliar por aeronaves comerciais;

- Modificação do modelo para inclusão de operações de aeronaves comerciais a partir da pista auxiliar, com o objetivo de determinar a nova capacidade do aeroporto nessa condição de operação;

- Elaboração de modelos mais abrangentes, capazes de verificar se o tráfego aéreo induzido pelos outros aeroportos localizados na TMA São Paulo (Guarulhos e Campinas) tem impacto negativo sobre a capacidade do Aeroporto de Congonhas. 


\section{REFERÊNCIAS}

AIRBUS - Societé par Actions Simplifiée. A319 Airplane Characteristics for Airport Planning. Toulouse, France, 1995.

AIRBUS - Societé par Actions Simplifiée. A320 Airplane Characteristics for Airport Planning. Toulouse, France, 1995b.

Alves, C. J. P. Uma Metodologia para Avaliação e Dimensionamento de Terminais de Passageiros em Aeroportos Brasileiros. 1981. Dissertação (Mestrado) - Escola Politécnica, Universidade de São Paulo, São Paulo, 1981.

ANAC - Agência Nacional de Aviação Civil. Ato Resolução 2, Diretoria, Brasília, 2006.

ANAC - Agência Nacional de Aviação Civil. Resolução 55, Diretoria, Brasília, 2008a.

ANAC - Agência Nacional de Aviação Civil. Instrução de Aviação Civil IAC 121-1013. Procedimentos e Requisitos Técnico-Operacionais Complementares para Operação no Aeroporto de Congonhas (Empresa Aérea RBHA 121), Superintendência de Segurança Operacional, Rio de Janeiro, 2008b.

ATR - Avions de Transport Régional. ATR 42 Airplane Characteristics. Toulouse, France, 1999.

BARRAGAN, G. A. D.; ALVES, C. J. P. Utilização de um Modelo de Simulação para a Análise da Capacidade do SBKP. In: Anais do VII SITRAER - Simpósio de Transporte Aéreo, Rio de Janeiro, 2008. v. 1, p. 925-936.

BARRAGAN, G. A. D. Uma Análise de Capacidade das Pistas dos Aeroportos da CTR São Paulo Através de Simulação. 2009. Dissertação (Mestrado), Instituto Tecnológico de Aeronáutica, São José dos Campos, 2009.

BASTOS, A. D.; BAUM, D. M.; CORREIA, A. R. Análise de Capacidade de Operação dos Boxes do Aeroporto de São Paulo - Congonhas por Meio de Simulação Computacional. In: Anais do VII SITRAER - Simpósio de Transporte Aéreo, Rio de Janeiro, 2008. v. 1, p. 193-208.

BASTOS, A. L. D. Uma Aplicação do Conceito de Navegação Baseado em Performance: Análise das Rotas ATS de Salvador. 2009. Dissertação (Mestrado), Instituto Tecnológico de Aeronáutica, São José dos Campos, 2009.

BAUM, D. M. Uma Análise da Carga de Trabalho do APP-SP por Meio de Simulação Computacional. 2009. Dissertação (Mestrado), Instituto Tecnológico de Aeronáutica, São José dos Campos, 2009.

BOEING Commercial Airplanes. 737 Airplane Characteristics for Airport Planning. Seattle, USA, 2005. 
BOEING Commercial Airplanes. Current Market Outlook 2010-2029. Seattle, USA, 2010.

CGNA - Centro de Gerenciamento de Navegação Aérea. Tabela de Alocação de Slots, , Rio de Janeiro, 2009. Disponível em http://www.cgna.gov.br/full_web_slot/. Último acesso: 25 de abril de 2009.

COMANDO DA AERONÁUTICA. Demanda Detalhada dos Aeroportos Brasileiros. Instituto de Aviação Civil, Rio de Janeiro, 2005.

COMANDO DA AERONÁUTICA. Circular de Tráfego Aéreo 100-6, Separação Radar Mínima de 3NM entre Aeronaves. Departamento de Controle do Espaço Aéreo, Rio de Janeiro, 2007.

COMANDO DA AERONÁUTICA. AIP-Brasil. Departamento de Controle do Espaço Aéreo, Rio de Janeiro, 2009a.

COMANDO DA AERONÁUTICA. ICA 100-12, Regras do Ar e Serviços de Tráfego Aéreo. Departamento de Controle do Espaço Aéreo, Rio de Janeiro, 2009b.

COMANDO DA AERONÁUTICA. NOTAM D0349/2009 e D0350/2009, 2009d. Disponível em http://www.aisweb.aer.mil.br/aisweb/. Último acesso: 10 de abril de 2009.

COMANDO DA AERONÁUTICA. AIP-MAP. Departamento de Controle do Espaço Aéreo, Rio de Janeiro, 2010.

DE NEUFVILLE, R. Airport Systems Planning - A Critical Look at the Methods and Experience. Center for Transportation Studies, Massachusetts Institute of Technology, The MacMillan Press, London, 1976.

DECEA - Departamento de Controle do Espaço Aéreo. AIC N 25/08 - Diretrizes Operacionais Relativas às Características do Vórtice da Esteira de Turbulência do Airbus A380-800, 2008. Disponível em http://publicacoes.decea.gov.br. Último acesso de 10 de setembro de 2010.

FAA - Federal Aviation Administration. Wake Turbulence Training Aid, 1995. Disponível em http://www.faa.gov/training_testing/training/media/wake/01wake.pdf. Último acesso em 07 de setembro de 2010.

FAA - Federal Aviation Administration. SIMMOD Manual How SIMMOD Works, 2003. Disponível em http://www.tc.faa.gov/acb300/how_AASW_works.pdf. Último acesso em 10 de abril de 2010.

FOKKER Aircraft B. V. Airplane Characteristics for Airport Planning. Schiphol, The Netherlands, 1990.

FRAGA, R. Analysis of Criteria for Closely-Spaced Parallel Runway Approaches Applied to a Multiple Airport System, 2009. Dissertação (Mestrado), Instituto Tecnológico de Aeronáutica, São José dos Campos, 2009. 
GUALDA, N. D. F. Modeling the Airport Terminal Building for Capacity Evaluation under Level-of-Service Criteria, 1978. PhD Dissertation, The University of Texas at Austin, Austin, TX, USA, 1978.

GUALDA, N. D. F. Terminais de Transportes: Contribuição ao Planejamento e ao Dimensionamento Operacional, 1995. Tese (Livre Docência), Escola Politécnica, Universidade de São Paulo, São Paulo, 1995.

GUALDA, N. D. F.; BOTTER, R. C.; RODRIGUES FILHO, O. S. Simulation-Based Analyses of Aircraft Passenger Bridges Installation at Sao Paulo Congonhas Airport. In: IX Air Transport Research Society World Conference ATRS, Rio de Janeiro, 2005. p. 1-9.

HORONJEFF, R. Planning and Design of Airports. McGraw Hill, $2^{\text {nd }}$ ed., New York, USA, 1975.

HORONJEFF, R.; MCKELVEY, F. X. Planning \& Design of Airports. McGraw Hill, $4^{\text {th }}$ ed, Boston, USA, 1994.

HUPALO, M. F. Análise da Área de Controle Terminal São Paulo (TMA-SP) por Meio de Simulação (Fast Time Simulation). 2003. Dissertação (Mestrado), Instituto Tecnológico de Aeronáutica, São José dos Campos, 2003.

ICAO - International Civil Aviation Organization. Annex 11 to the Convention on International Civil Aviation - Air Traffic Services. International Civil Aviation Organization, $13^{\text {th }}$ ed., Montreal, Canadá, 2001.

ICAO - International Civil Aviation Organization. Annex 2 to the Convention on International Civil Aviation - Rules of the Air. International Civil Aviation Organization, $10^{\text {th }}$ ed., Montreal, Canadá, 2005.

ICAO - International Civil Aviation Organization. Doc 7300/9 - Convention on International Civil Aviation. International Civil Aviation Organization, $9^{\text {th }}$ ed., Montreal, Canadá, 2006.

ICAO - International Civil Aviation Organization. Doc 4444 ATM/501 - Procedures for Air Navigation Services - Air Traffic Management. International Civil Aviation Organization, $15^{\text {th }}$ ed., Montreal, Canadá, 2007.

ICAO - International Civil Aviation Organization. Doc 7030 - Regional Supplementary Procedures. International Civil Aviation Organization, $5^{\text {th }}$ ed., Montreal, Canadá, 2008.

INFRAERO - Empresa Brasileira de Infra-estrutura Aeroportuária. Histórico do Aeroporto de Congonhas, 2010a. Disponível em http://www.infraero.gov.br/index.php/br/aeroportos/sao-paulo/aeroporto-de-saopaulo--congonhas/historico.html. Último acesso de 27 de setembro de 2010. 
INFRAERO - Empresa Brasileira de Infra-estrutura Aeroportuária. Movimento Operacional Acumulado, 2010b. Disponível em http://www.infraero.gov.br/movi.php. Último acesso em 27 de setembro de 2010.

ISA Software. RAMS Plus Simulation Solutions. 2009. Disponível em http://www.ramsplus.com/files/What\%20ls\%20RAMS\%20Plus.pdf. Último acesso em 18 de setembro de 2010.

JEPPESEN A Boeing Company. TAAM Solutions. Jeppesen, 2010a. Disponível em http://www.jeppesen.com/industry-solutions/aviation/government/total-airspaceairport-modeler.jsp. Último acesso em 18 de setembro de 2010.

JEPPESEN A Boeing Company. South America Airway Manual. 2010b, Englewood, CO, USA.

JEPPESEN A Boeing Company. United States Airway Manual. 2010c, Englewood, CO, USA.

KAZDA, A., CAVES, R. E. Airport Design and Operation. Emerald Group Publishing Limited, $2^{\text {nd }}$ ed, Howard House, Wagon Lane, Bingley, UK, 2008.

MEREDITH, D. D.; WONG, K. W.; WORTMAN, R. H. Design and Planning of Engineering Systems. Prentice-Hall Inc., New Jersey, USA, 1973.

METROPOLITAN TRANSPORTATION COMISSION (MTC). Regional Airport System Plan Update 2000 Final Report, San Francisco, CA, USA, 2000.

MOSER, R. F. Simulação e Análises de Configurações Aeroportuárias Utilizando Visual SIMMOD: Aplicação ao Aeroporto Internacional de São Paulo / Guarulhos. 2007. Dissertação (Mestrado), Escola Politécnica, Universidade de São Paulo, São Paulo, 2007.

MÜLLER, C. A Framework for Quality of Service Evaluation at Airport Terminals. 1987. Tese (Doutorado), University of California, Berkeley, CA, USA, 1987.

MÜLLER, C; SANTANA, E. S. M. Analysis of flight-operating costs and delays: The Sao Paulo terminal maneuvering area. Journal of Air Transport Management, 2008. v. 14, n. 6, p. $293-296$.

ODONI, A. R. et al. Existing and Required Modeling Capabilities for Evaluating ATM Systems and Concepts. International Center for Air Transportation, Massachussets Intitute of Technology, 1997. Disponível em

http://web.mit.edu/aeroastro/www/labs/AATT/aatt.html. Último acesso de 18 de setembro de 2010.

OLIVEIRA, M. Carga de Trabalho dos Controladores de Tráfego Aéreo - Análise da Área de Controle Terminal Brasília por Meio de Simulação. 2007. Dissertação (Mestrado), Instituto Tecnológico de Aeronáutica, São José dos Campos, 2007. 
ORTÚZAR, J. D.; WILLUMSEN, L. G. Modeling Transport. John Willey \& Sons, $3^{\text {rd }}$ ed., New York, USA, 2001.

PIDD, M. Just Modeling Through: A Rough Guide to Modeling. Interfaces, 1999. v. 29 , n. 2, p. 118-132.

PERRELLA, A. C. F. et al. Comparação dos Elementos Climáticos Observados nas Estações dos Aeroportos de Guarulhos, Congonhas, Campinas e São José dos Campos. XI Congresso Brasileiro de Meteorologia, Rio de Janeiro, 2000, v. 1, p. 804 $-811$.

POGIANELO, M. Análise do Espaço Aéreo Brasileiro por Meio de Simulação "Fast Time" - Estudo de Caso na Área de Controle Terminal Recife. 2008. Dissertação (Mestrado), Instituto Tecnológico de Aeronáutica, São José dos Campos, 2008.

PRADO, D. Usando o Arena em Simulação. INDG Tecnologia e Serviços Ltda, $1^{\text {a }}$ ed., Belo Horizonte, 2003.

RIBEIRO, F. R. Modelo de Simulação para Análise Operacional de Pátios de Aeroportos. 2003. Dissertação (Mestrado), Escola Politécnica, Universidade de São Paulo, São Paulo, 2003.

SANTANA, E. S. M. Análise de Novos Cenários Operacionais para o Aeroporto Internacional de São Paulo / Guarulhos. 2002. Dissertação (Mestrado), Instituto Tecnológico de Aeronáutica, São José dos Campos, 2002.

SOARES, A. C. F. Regulamento de Tráfego Aéreo para Piloto Privado de Avião e Helicóptero, Manual Didático para o Voo Visual (VFR). Espaço Aéreo, $7^{\text {a }}$ ed., São Paulo, 1993.

SUBRAMANIAN, P. A. Simulation Study to Investigate Runway Capacity Using TAAM. 2002. Dissertação (Master Business Administration), Embry-Riddle Aeronautical University, Daytona Beach, Florida, USA, 2002.

SWEDISH, W. J. Upgraded FAA Airfield Capacity Model Volume I: Supplemental User's Guide. The MITRE Corporation, Virginia, USA, 1981.

WINSTON L. W. Operations Research Applications and Algorithms. Thompson Brooks / Cole, $4^{\text {th }}$ ed, Belmont, CA, USA, 2004. 


\section{APÊNDICE A - FUNCIONAMENTO DO SOFTWARE SIMMOD}

O SIMMOD é um modelo de simulação de eventos discretos e representa um sistema evoluindo no tempo, por meio de um modelo matemático e variáveis, cujo estado varia em pontos discretos do tempo. Esses pontos, conhecidos como eventos, são momentos onde ocorrem mudanças nas variáveis de estado. Por exemplo: considerando uma fila simples de aeronaves aguardando para decolagem, a chegada de uma nova aeronave na fila é um evento. O SIMMOD, então, calcula o efeito desse evento no comportamento do sistema e modifica o estado das variáveis afetadas antes de processar o próximo evento. Trata-se, também, de um modelo estocástico que utiliza variáveis aleatórias para produzir resultados únicos a cada iteração, representando as variações diárias do tráfego aéreo.

O escalonador de eventos e o relógio de simulação trabalham juntos para processar os eventos na sequência apropriada. O relógio avança de um evento para outro e o modelo ignora os intervalos de tempo onde não ocorrem eventos. Os eventos são processados na ordem estrita em que aparecem no escalonador. Se dois ou mais eventos são programados para ocorrer no mesmo instante de tempo, o relógio de simulação não avança até que todos tenham sido processados. No início da simulação, o SIMMOD inicializa o relógio com 00:00 e ordena os eventos externos. O relógio, então, avança para o primeiro evento e atualiza o estado das variáveis do sistema. Em seguida, o relógio avança para o segundo evento e o processo se repete até o final da simulação.

Para representar aeroportos e espaço aéreo o SIMMOD utiliza nós conectados por arcos. Um nó é um ponto do sistema onde o SIMMOD avalia a posição de uma aeronave com relação às demais aeronaves do sistema. Um arco define o caminho entre dois nós, por onde as aeronaves se movem. Existem dois grupos diferentes de nós: terrestres (ground nodes) e aéreos (airspace nodes). Nós terrestres representam posições no aeroporto, como gates, filas de decolagem e intersecções de pistas de pouso e de táxi. Nós aéreos representam locais como auxílios rádio à navegação, fixos, órbitas de espera, junções de rotas e interfaces com aeroportos. Os arcos terrestres e aéreos também formam dois grupos separados: os arcos terrestres representam pistas de pouso e táxi, enquanto os arcos aéreos 
representam rotas, incluindo subidas, chegadas e aerovias. Grandes estruturas, como rotas e pistas, são geralmente compostas por vários arcos.

Cada voo criado na simulação corresponde a um certo tipo de aeronave. Os dados da aeronave permitem ao sistema distinguir diferentes aeronaves e atribuir regras apropriadas de separação, sequenciamento, velocidades, características de decolagem e pouso e limitações baseadas em tamanho da aeronave para cada um dos voos simulados.

O espaço aéreo simulado no SIMMOD é composto de uma malha de rotas das aeronaves. As rotas são definidas como uma série de nós e arcos e, quando duas ou mais rotas convergem, alguns nós e arcos serão comuns, ou seja, poderão aparecer nas definições de mais de uma rota. Todas as aeronaves movem-se no espaço ao longo dessas rotas e todos os voos simulados devem ter uma rota pré definida nos dados de entrada.

A cada movimento de uma aeronave no espaço aéreo simulado, os requisitos de separação são checados em relação a outras aeronaves na mesma rota e em rotas que se juntam ou que se cruzam. Ao contrário de voos reais, as aeronaves na simulação não podem desviar-se das rotas previamente definidas. Se isso ocorresse, os mínimos de separação não poderiam ser checados a cada iteração. A separação lateral é mantida, considerando-se que as rotas de entrada foram corretamente definidas.

Cada nó em uma rota tem uma altitude pré-definida, que é usada para calcular o consumo de combustível e a velocidade verdadeira. As altitudes são checadas e, caso necessário, ajustadas para manter as separações verticais mínimas e resolver conflitos.

Uma vez que o SIMMOD foi projetado para fornecer resultados realistas a partir de qualquer iteração de um conjunto de dados de entrada, é necessário rodar diversas iterações com um conjunto único de dados de entrada para estabelecer tendências estatisticamente significativas. Para uma série de iterações, os relatórios de saída agregam valores, além de calcular médias e desvios padrão, quando apropriado. 
Outros trabalhos existentes na literatura mostram que dez iterações são suficientes para produzir resultados confiáveis com um conjunto de dados de entrada (SANTANA, 2002 e MOSER, 2007). 


\section{APÊNDICE B - VALIDAÇÃO DO MODELO DE SIMULAÇÃO}

A validação do modelo de simulação foi feita comparando-se os movimentos previstos com os realizados em cada hora de funcionamento do aeroporto, no período escolhido. Para o modelo de Congonhas, a tabela 14 apresenta 0 comparativo.

Tabela 14 - Movimentos previstos e realizados no cenário de validação

\begin{tabular}{|c|c|c|c|c|c|c|c|c|c|}
\hline Hora & $\begin{array}{l}\text { DEP } \\
\text { Prev }\end{array}$ & $\begin{array}{l}\text { DEP } \\
\text { Simul }\end{array}$ & $\begin{array}{c}\text { Dif } \\
\text { DEP }\end{array}$ & $\begin{array}{l}\text { ARR } \\
\text { Prev }\end{array}$ & $\begin{array}{l}\text { ARR } \\
\text { Simul }\end{array}$ & $\begin{array}{c}\text { Dif } \\
\text { ARR }\end{array}$ & $\begin{array}{l}\text { Total } \\
\text { Prev }\end{array}$ & $\begin{array}{l}\text { Total } \\
\text { Simul }\end{array}$ & $\begin{array}{c}\text { Dif } \\
\text { Total }\end{array}$ \\
\hline $06-07$ & 17 & 16 & 1 & 11 & 11 & 0 & 28 & 27 & 1 \\
\hline 07-08 & 12 & 11 & 1 & 17 & 17 & 0 & 29 & 28 & 1 \\
\hline 08-09 & 19 & 21 & -2 & 12 & 12 & 0 & 31 & 33 & -2 \\
\hline $09-10$ & 11 & 12 & -1 & 19 & 20 & -1 & 30 & 32 & -2 \\
\hline $10-11$ & 18 & 17 & 1 & 13 & 13 & 0 & 31 & 30 & 1 \\
\hline $11-12$ & 14 & 13 & 1 & 16 & 15 & 1 & 30 & 28 & 2 \\
\hline $12-13$ & 14 & 14 & 0 & 15 & 14 & 1 & 29 & 28 & 1 \\
\hline $13-14$ & 17 & 16 & 1 & 13 & 14 & -1 & 30 & 30 & 0 \\
\hline $14-15$ & 11 & 13 & -2 & 21 & 20 & 1 & 32 & 33 & -1 \\
\hline $15-16$ & 17 & 16 & 1 & 10 & 11 & -1 & 27 & 27 & 0 \\
\hline $16-17$ & 12 & 14 & -2 & 18 & 17 & 1 & 30 & 31 & -1 \\
\hline $17-18$ & 16 & 16 & 0 & 15 & 16 & -1 & 31 & 32 & -1 \\
\hline $18-19$ & 16 & 17 & -1 & 14 & 14 & 0 & 30 & 31 & -1 \\
\hline $19-20$ & 15 & 11 & 4 & 16 & 16 & 0 & 31 & 27 & 4 \\
\hline $20-21$ & 14 & 16 & -2 & 17 & 15 & 2 & 31 & 31 & 0 \\
\hline 21-22 & 14 & 15 & -1 & 18 & 20 & -2 & 32 & 35 & -3 \\
\hline $22-23$ & 11 & 10 & 1 & 6 & 6 & 0 & 17 & 16 & 1 \\
\hline Total & 248 & 248 & 0 & 251 & 251 & 0 & 499 & 499 & 0 \\
\hline
\end{tabular}

Com os dados da tabela 13, pode-se obter o gráfico da figura 29 , onde são mostradas as diferenças entre os movimentos de decolagem e pouso previstos e realizados por hora. A partir dessas diferenças, pode-se calcular a aderência média do modelo, por meio da equação 6. 


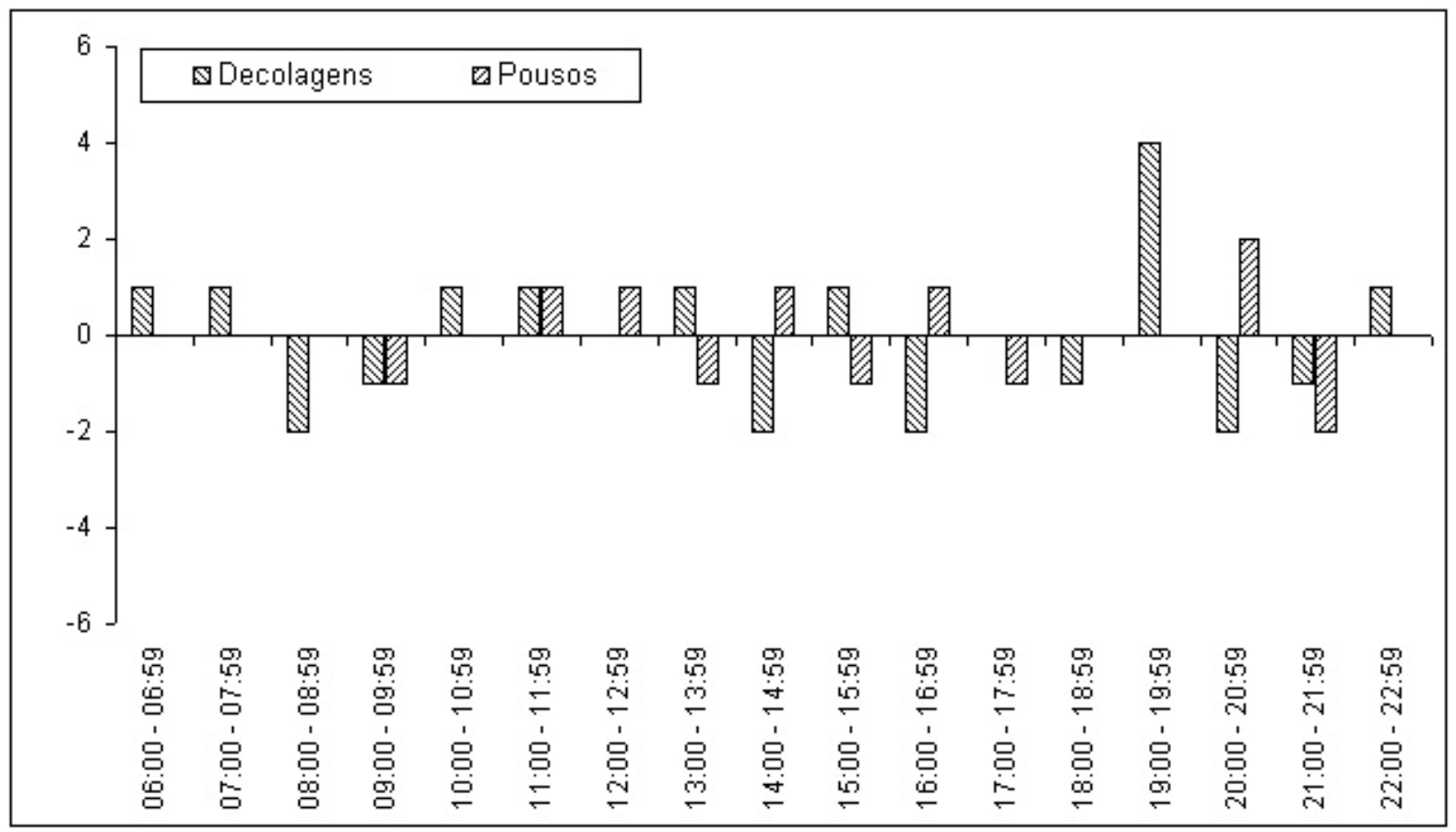

Figura 29 - Diferenças entre movimentos previstos e realizados no cenário de validação

$$
A=1-\frac{\sum_{1}^{h}\left|\frac{\text { Dif Total }}{\text { Total Prev }}\right|}{h}
$$

onde:

$A$ - aderência média do modelo

Dif Total - diferença entre movimentos previstos e realizados

Tot Prev - total de movimentos por hora

$h$ - horas de operação do aeroporto

Aplicando-se os dados da tabela 13 na equação 6, obtém-se o valor de 95,6\% de aderência do modelo criado à operação real do Aeroporto de Congonhas. 


\section{Anexo A - Saídas (SID) e Chegadas (STAR) de Congonhas}

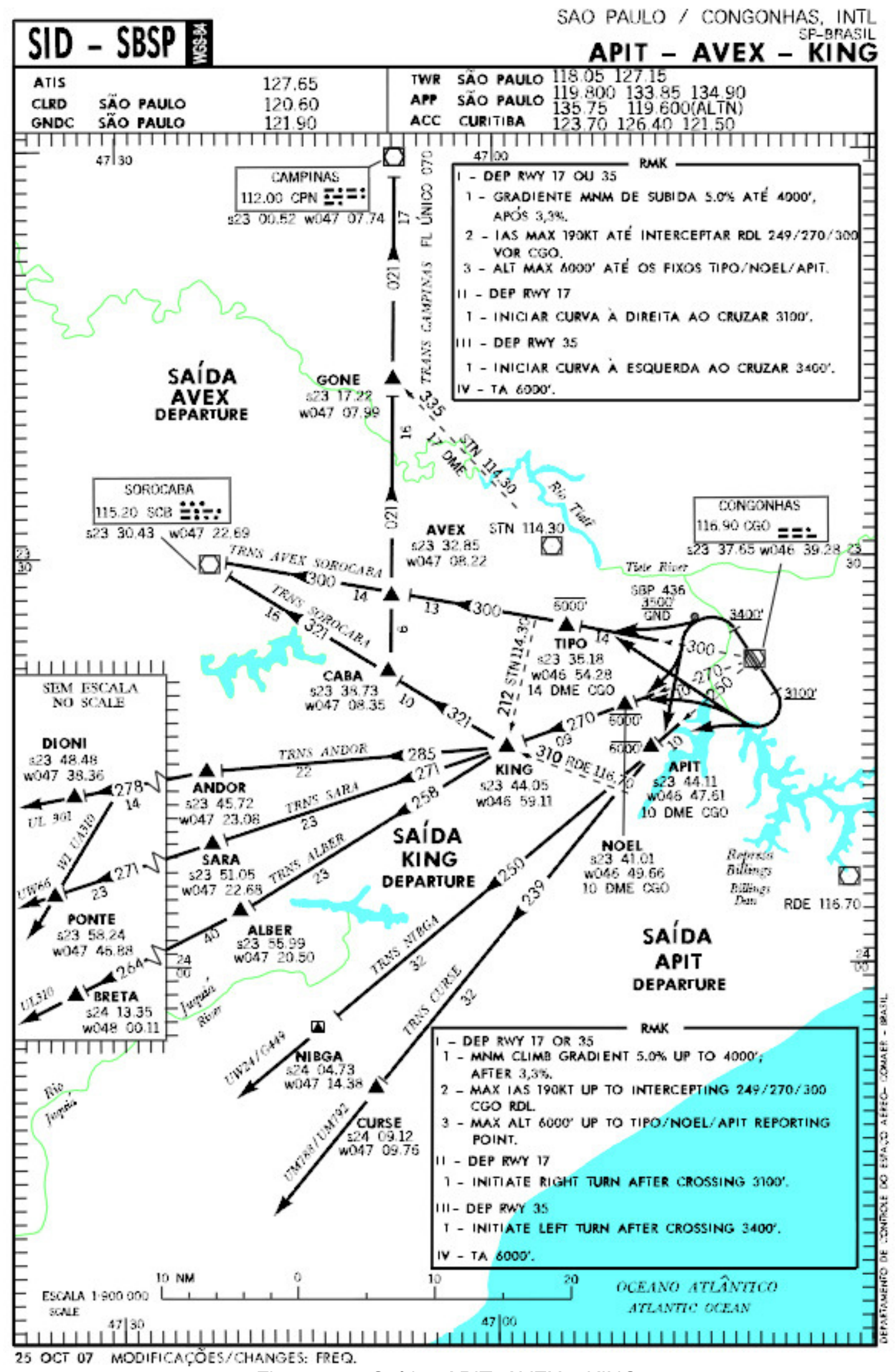

Figura 30 - Saídas APIT, AVEX e KING

Fonte: (COMANDO DA AERONÁUTICA, 2009) 


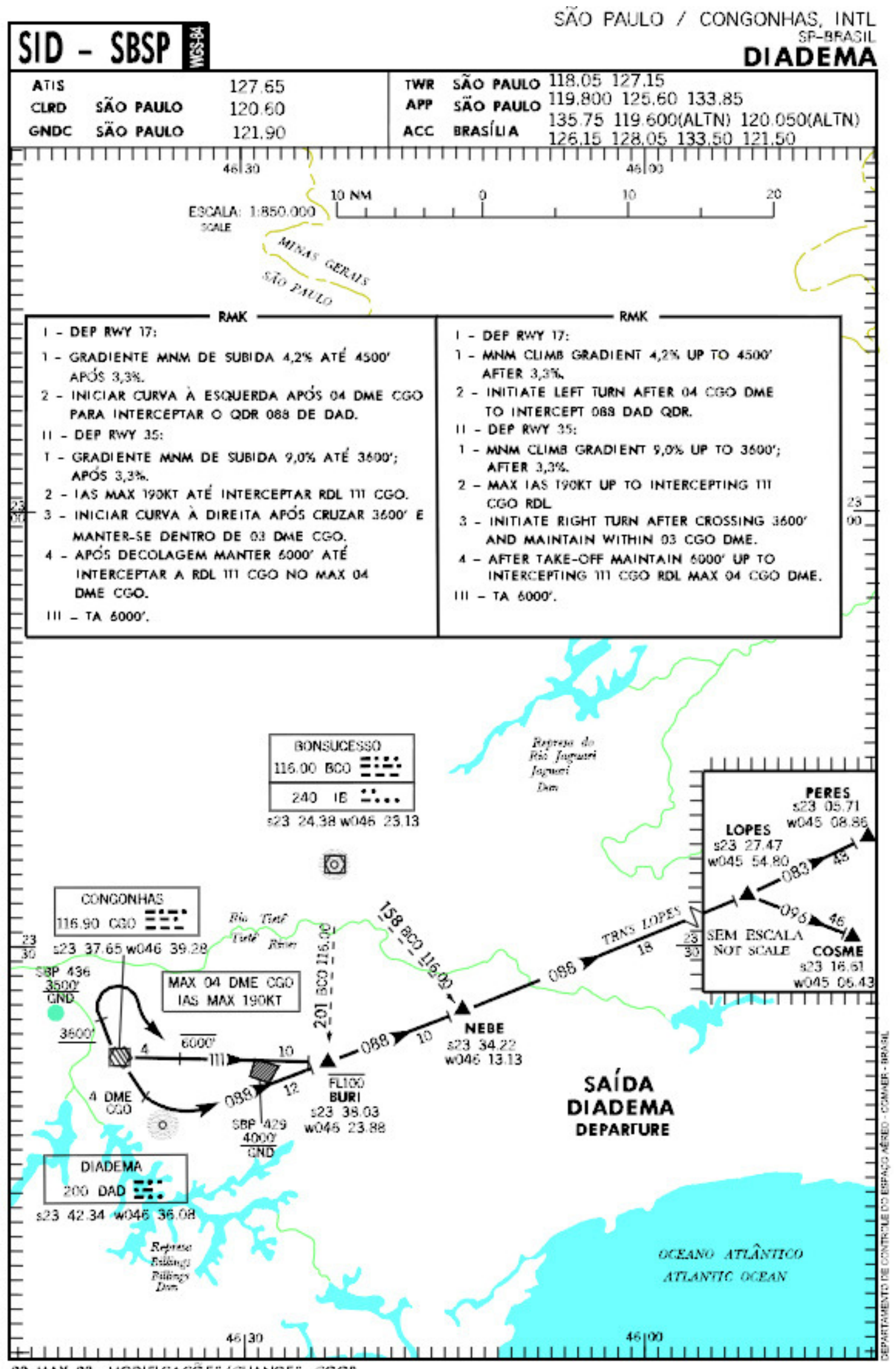

o8 MAY o8 MOOIFICACOES/CHANGES: COOR.

Figura 31 - Saída DIADEMA

Fonte: (COMANDO DA AERONÁUTICA, 2010) 


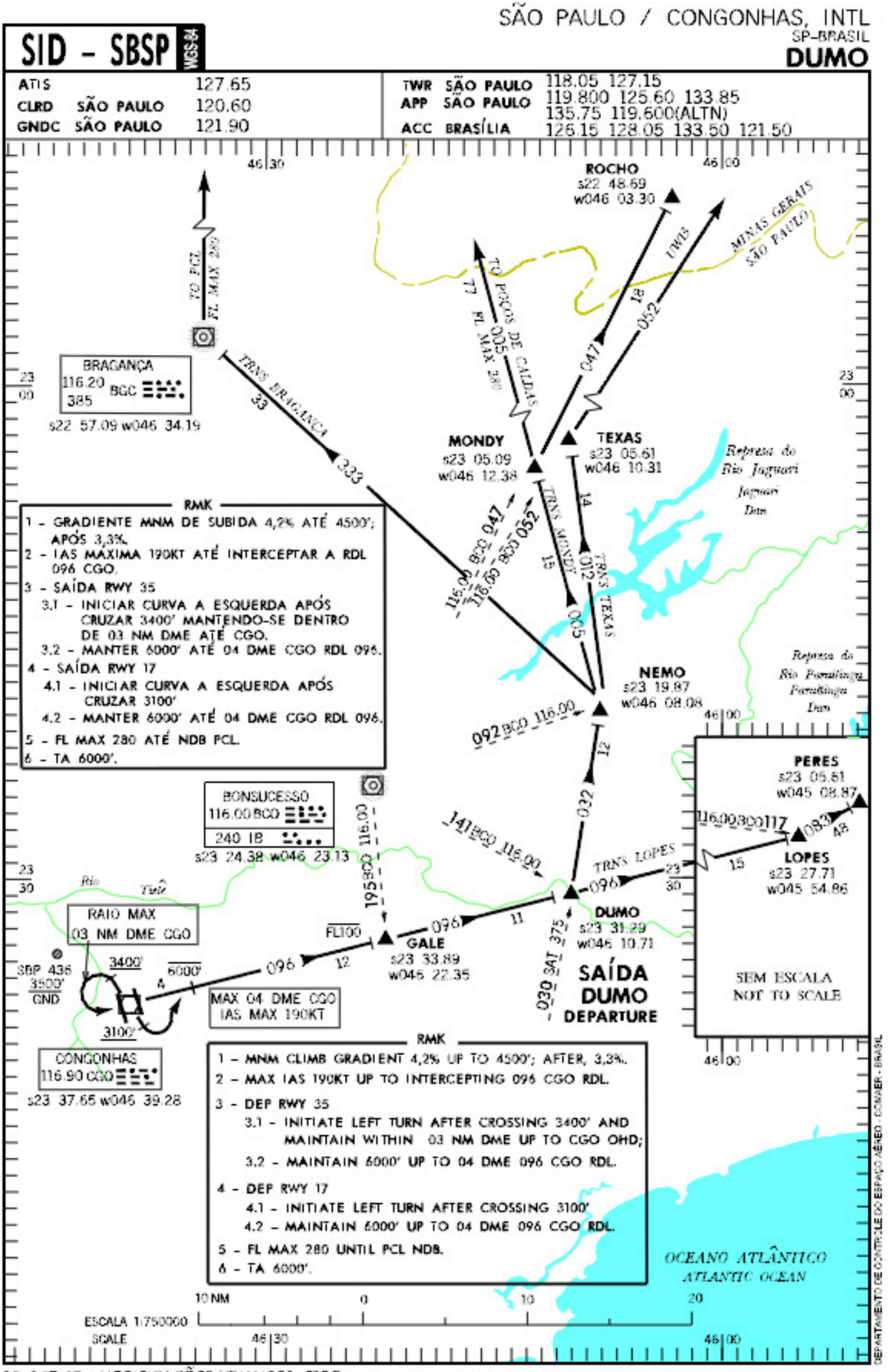

25 OCT O7 MODIFICAÇÕES/CHANGES: FREQ.

Figura 32 - Saída DUMO

Fonte: (COMANDO DA AERONÁUTICA, 2010) 


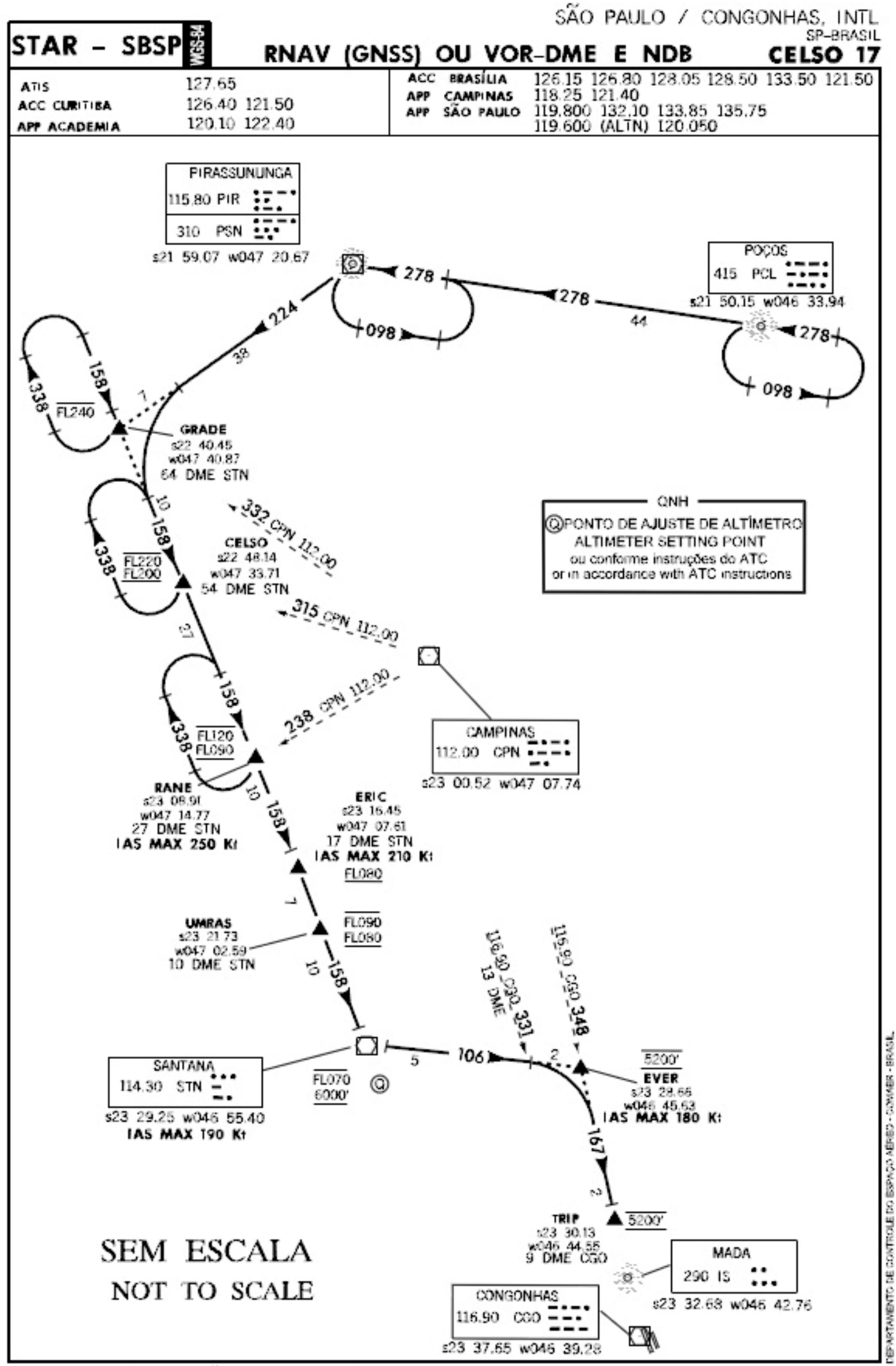

25 SEP O8 MODIFICACÓES/CHANCES: RMK.

Figura 33 - Chegada CELSO 17

Fonte: (COMANDO DA AERONÁUTICA, 2010) 


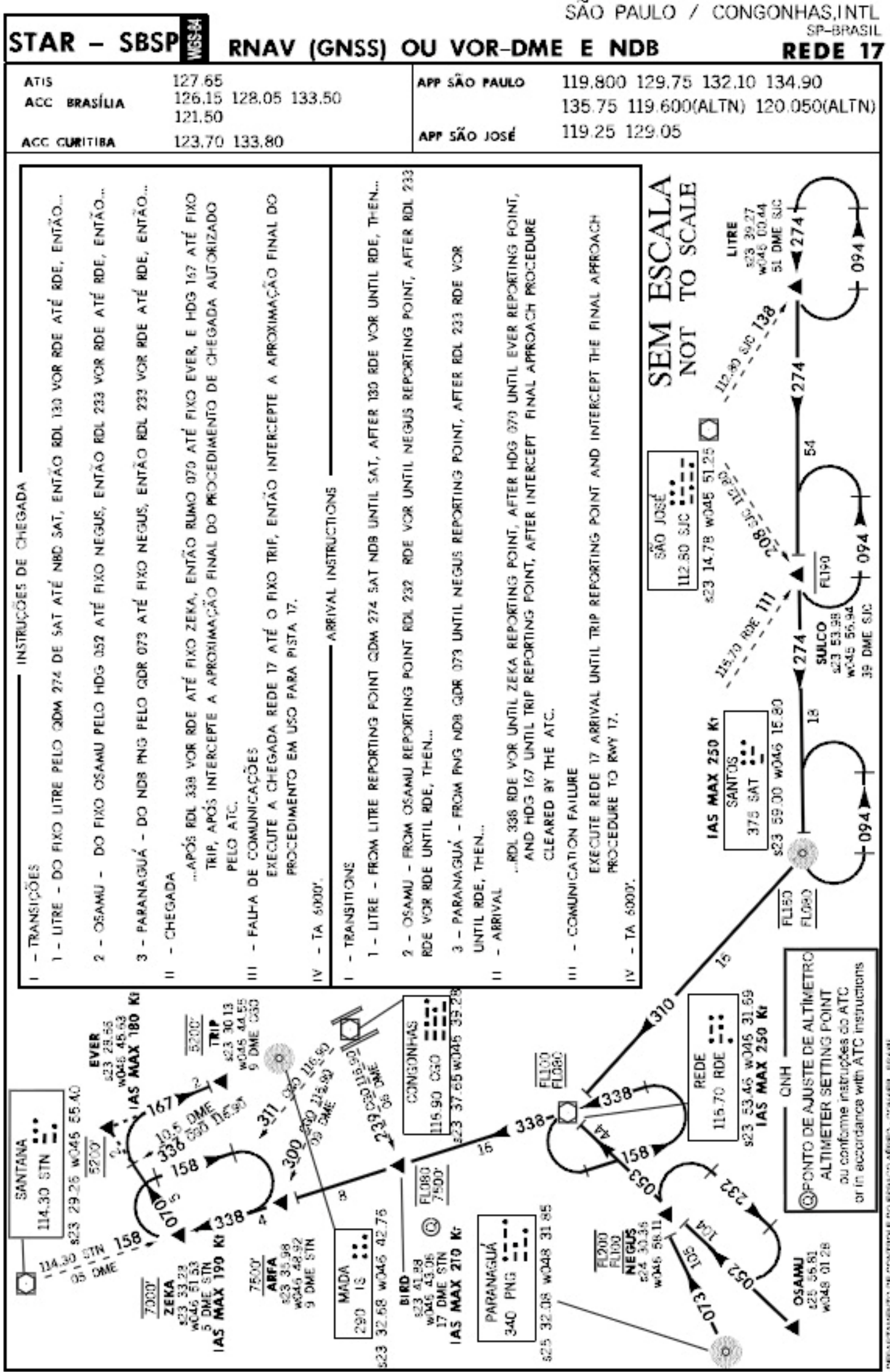

SÄO PAULO / CONGONHAS,INTL

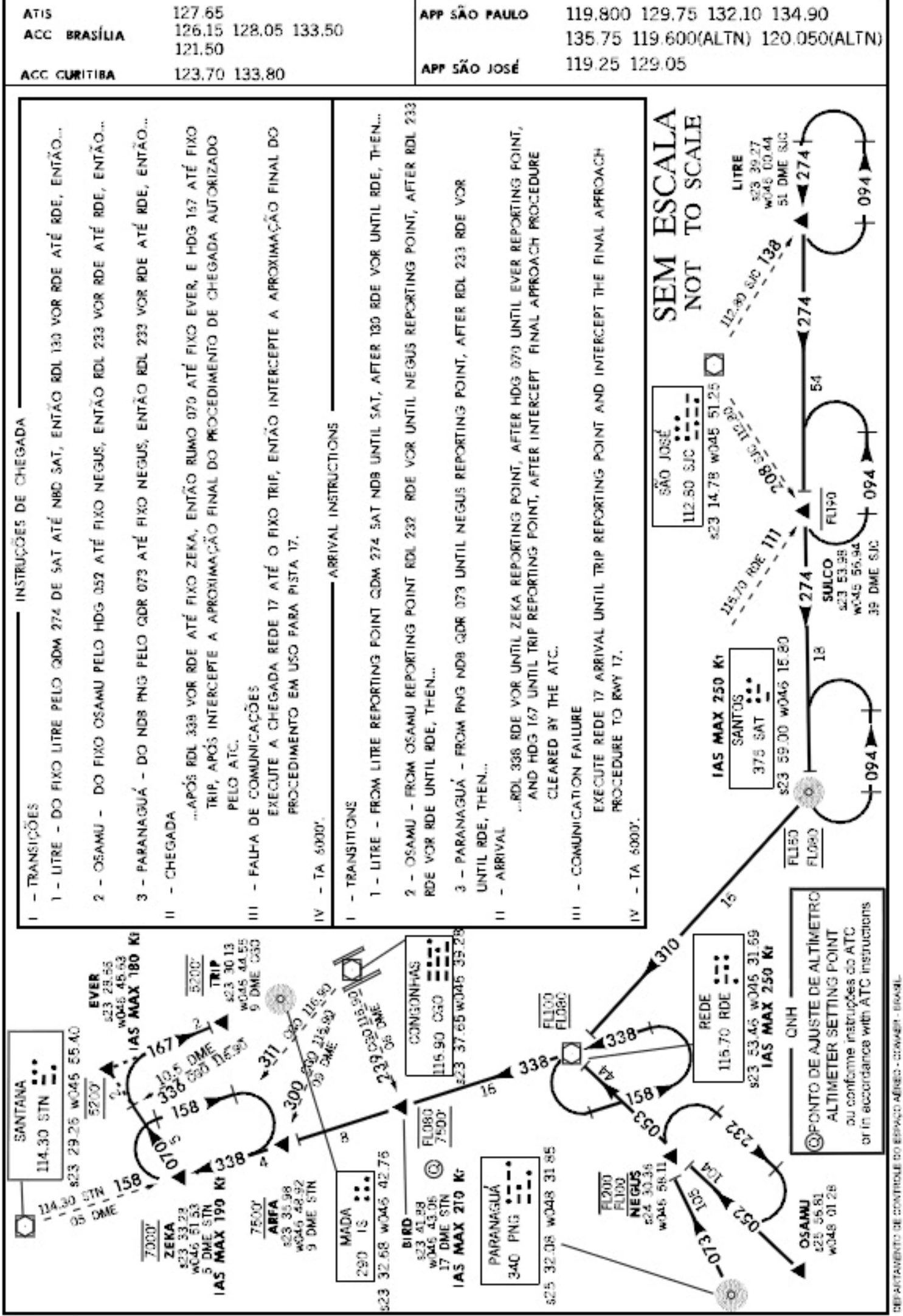

28 OCT O6 MODIFICAÇOES/CHANGES: COCRD.

Figura 34 - Chegada REDE 17

Fonte: (COMANDO DA AERONÁUTICA, 2010) 


\section{Anexo B - Diagramas de Ocupação do Estacionamento}

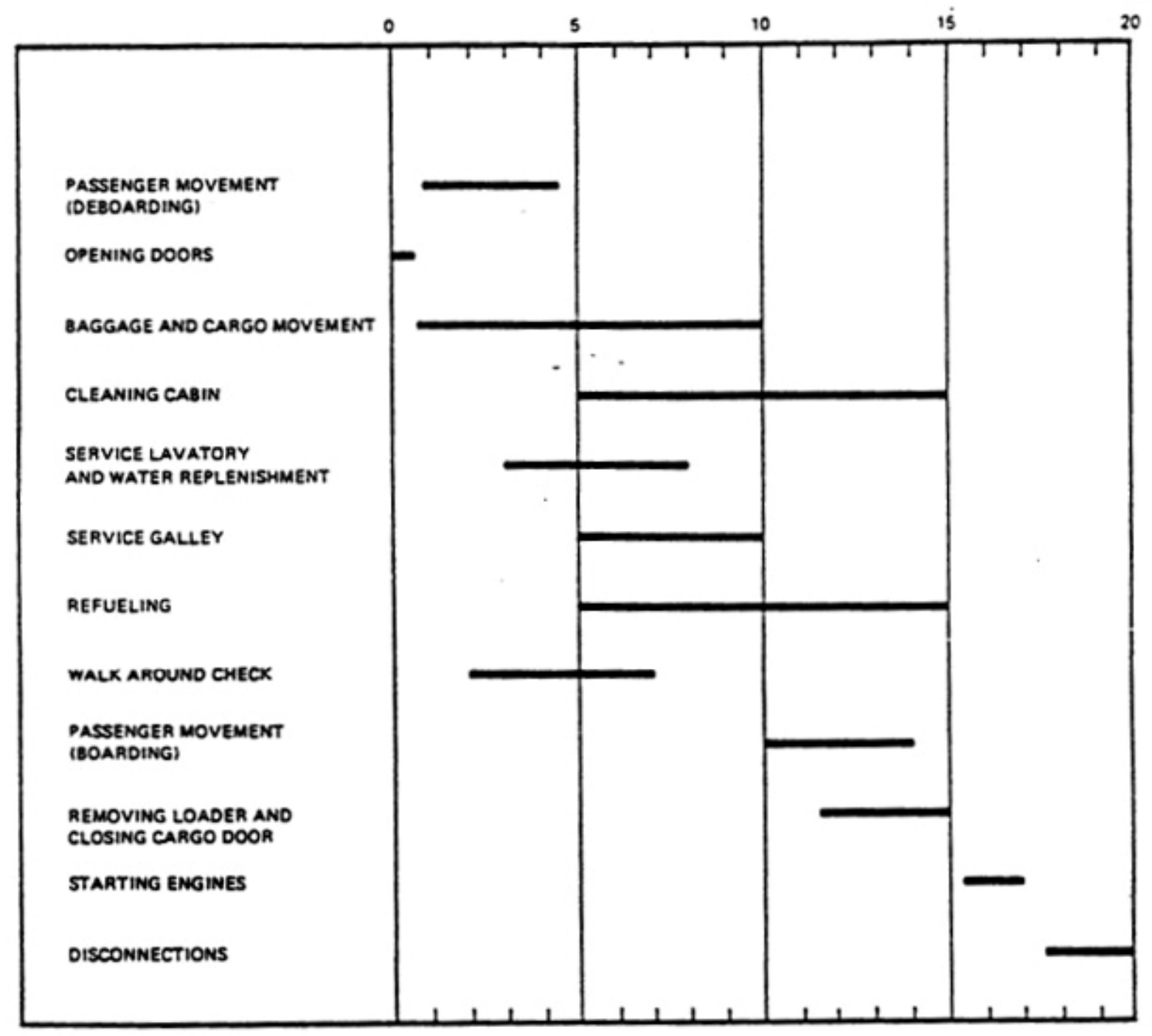

Figura 35 - Turn Around Time para aeronaves ATR 42-300 Fonte: (ATR, 1999) 


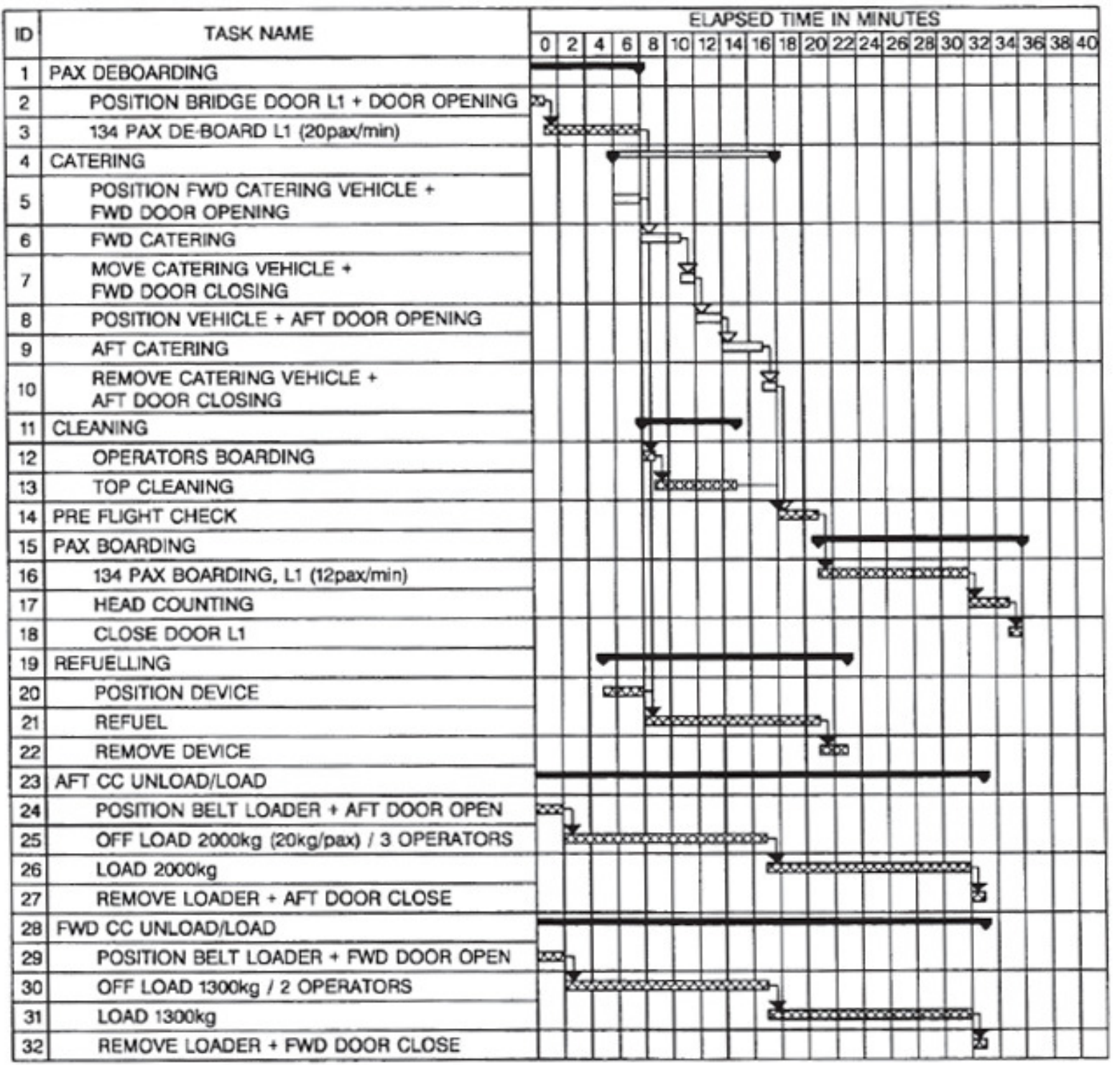

Figura 36 - Turn Around Time para aeronaves Airbus A319

Fonte: (AIRBUS, 1995) 


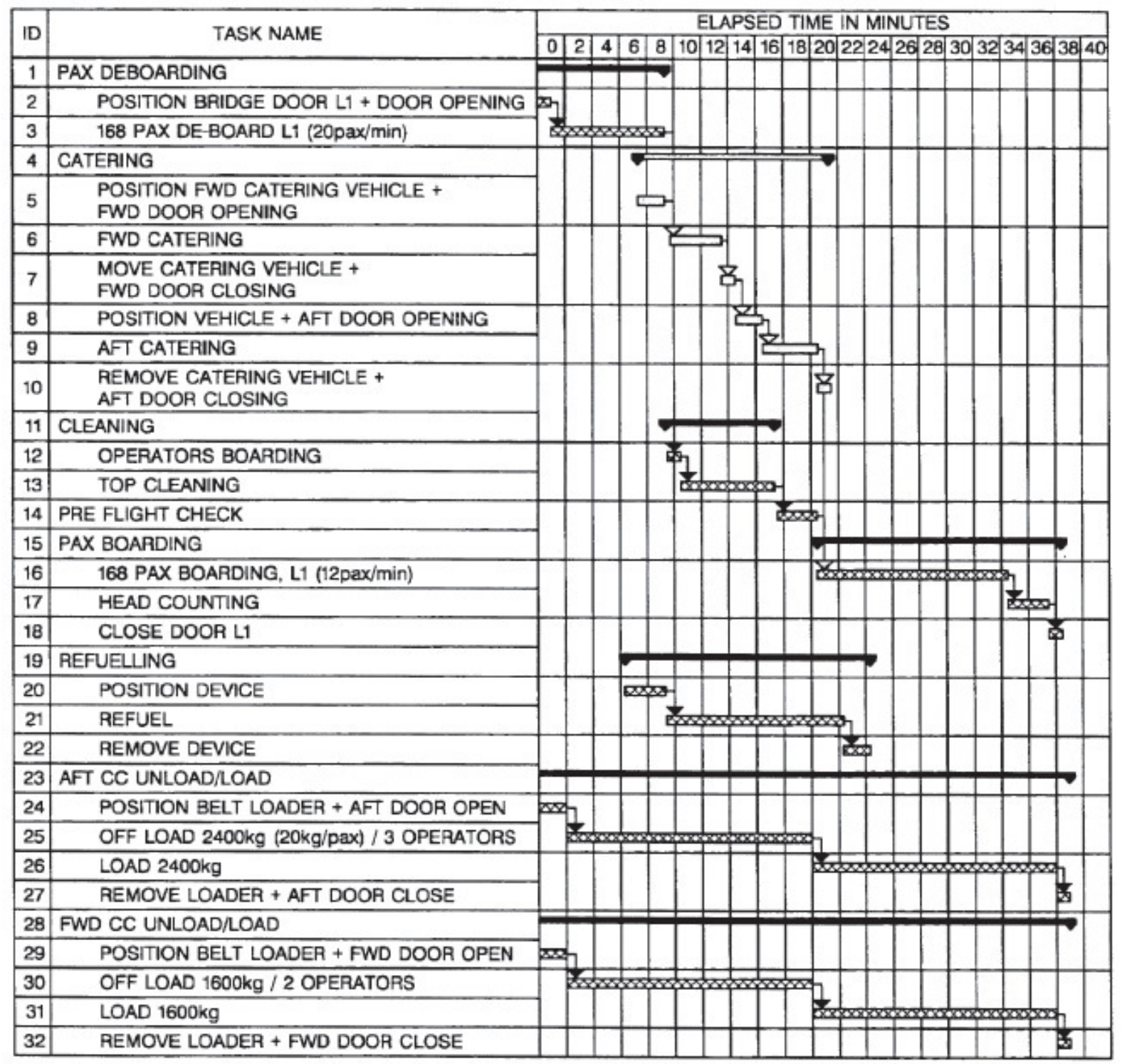

Figura 37 - Turn Around Time para aeronaves Airbus A320 Fonte: (AIRBUS, 1995b) 


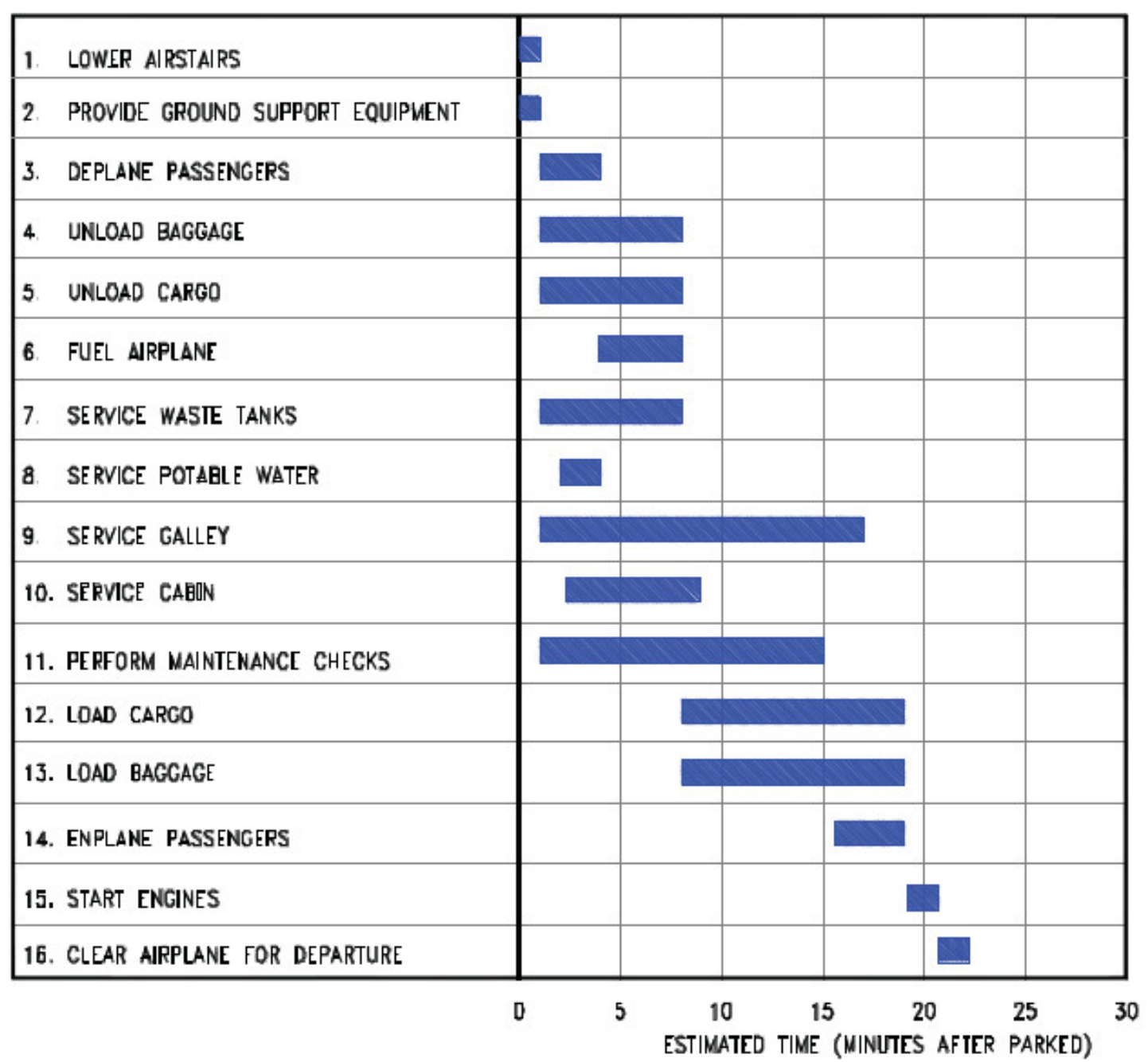

NOUT:

1. ESTIMATES BASED ON MIXED-CLASS CONFIGURATON, 65\% LOAD TACTOR

2. IT IS ASSUMED THAT ALL EQUIPMENT FUNTION PROPERLY AND THAT NO ABNORMAL WEATHER CONDDIONS EXIST,

3. TOTAL TIME ON THE RAMP IS 25 MINUTES

4. BOTH FORWARO AND AFT DOORS $\triangle R E$ USED

5. 75\% PASSENGER EXCHANGE

6. THIS DATA IS PROVDDED TO ILIUSTRATE IHE GENERAL SCOPE AND TYPES OF TASKS INVOLVED IN TERMINAL OPERATIONS. VARYING AIRLINE PRACTCES AND OPERATNNG CIRCUMSTANCES THROUGHOUT THE WORLD WILL RESULT IN DIFFERENT SEQUENGES AND TIME INTERVALS TO ACCOMPLISH THE TASKS SHOWN.

7. GROUND OPERATIONS REQUIREMENTS SHOULD BE COORDINATED WITH USING AIRLINES PRIOR TOO RAMP PLANNING

Figura 38 - Turn Around Time para aeronaves Boeing 737-100/200/300/400/500 Fonte: (BOEING, 2005) 


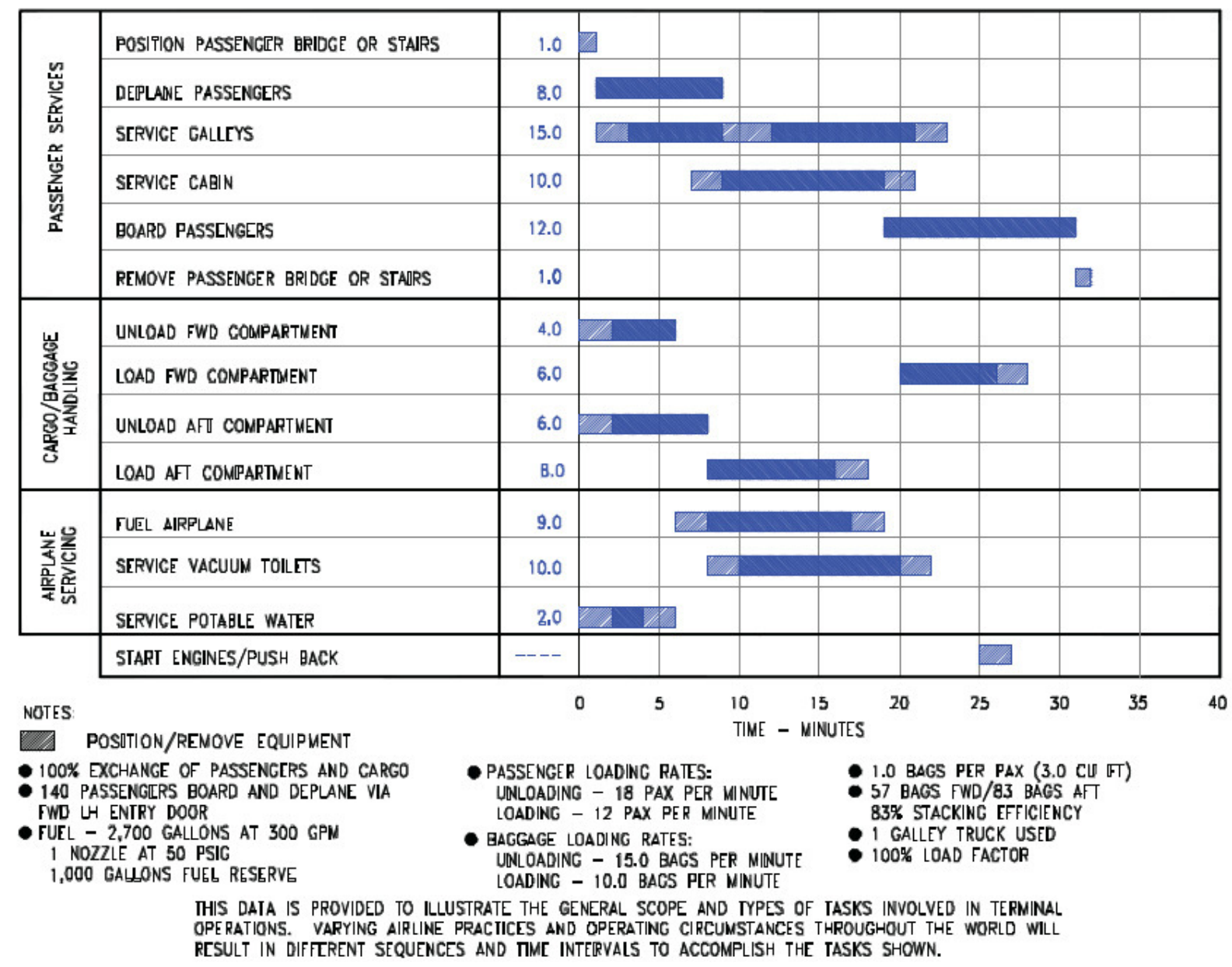

Figura 39 - Turn Around Time para aeronaves Boeing 737-700

Fonte: (BOEING, 2005) 


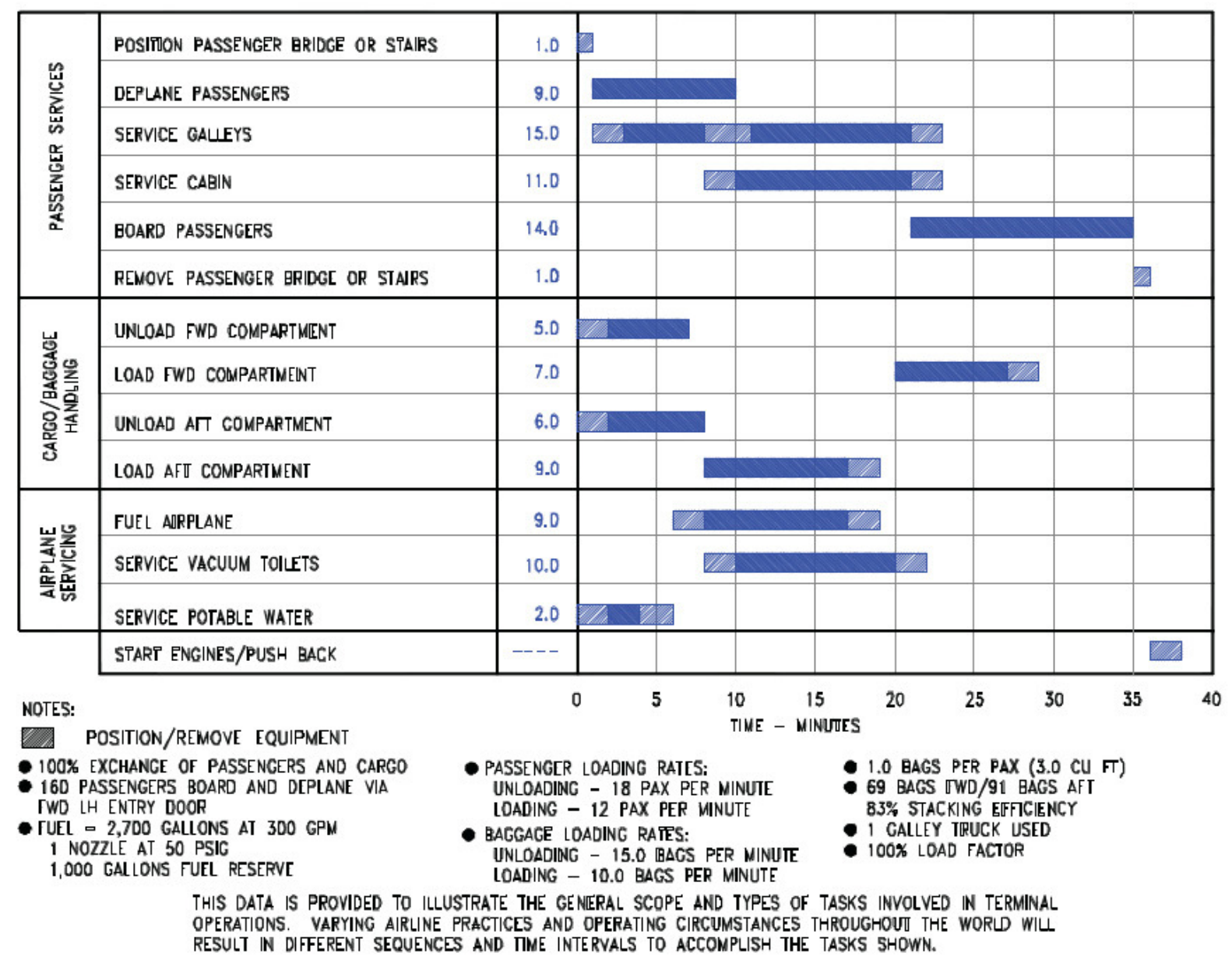

Figura 40 - Turn Around Time para aeronaves Boeing 737-800

Fonte: (BOEING, 2005) 


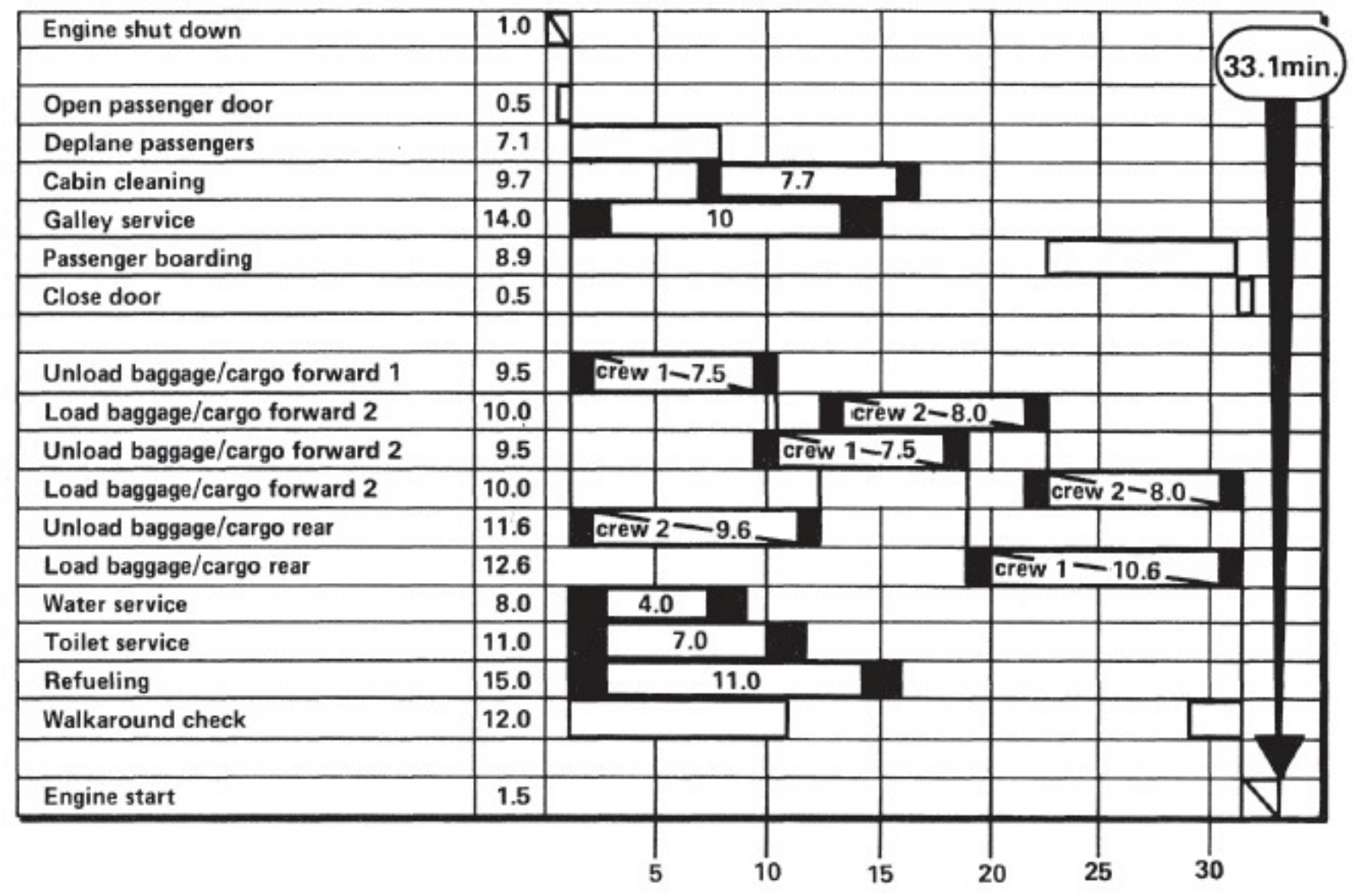

Figura 41 - Turn Around Time para aeronaves Fokker 100 Fonte: (FOKKER, 1990) 


\section{ANEXO C - Tabelas de Voos Simulados}

\section{C.1 - Indicadores de Localidades}

\begin{tabular}{ll}
\hline Código & Aeroporto \\
\hline SBAU & Araçatuba / SP \\
SBBR & Brasília / DF \\
SBCF & Belo Horizonte / MG \\
SBCG & Campo Grande / MS \\
SBCT & Curitiba / PR \\
SBCX & Caxias do Sul / RS \\
SBCY & Cuiabá / MT \\
SBDN & Presidente Prudente / SP \\
SBFL & Florianópolis / SC \\
SBGL & Rio de Janeiro / RJ (Antônio Carlos Jobim) \\
SBGO & Gioânia / GO \\
SBIL & Ilhéus / BA \\
SBJF & Juiz de Fora / MG \\
SBJV & Joinville / SC \\
SBLO & Londrina / PR \\
SBMG & Maringá / PR \\
SBML & Marília / SP \\
SBNF & Navegantes / SC \\
SBPA & Porto Alegre / RS \\
SBRJ & Rio de Janeiro / RJ (Santos Dumont) \\
SBRP & Ribeirão Preto / SP \\
SBSR & São José do Rio Preto / SP \\
SBSV & Salvador / BA \\
SBUL & Uberlândia / MG \\
SBVT & Vitória / ES \\
SJTC & Bauru / SP \\
\hline &
\end{tabular}


C.2 - Alocação de slots para voos comerciais em Congonhas

\begin{tabular}{|c|c|c|c|c|c|c|c|}
\hline Slot ATC & Voo & Origem & Destino & Eqpto & Tipo & Procedimento & Transição \\
\hline $6: 00$ & TAM3775 & SBCG & & A319 & ARR & CELSO 17 & GRADE \\
\hline $6: 03$ & GLO1563 & SBRJ & & B737 & ARR & REDE 17 & LITRE \\
\hline $6: 05$ & GLO1383 & SBGL & & B737 & ARR & REDE 17 & LITRE \\
\hline $6: 07$ & TAM3801 & SBCY & & А320 & ARR & CELSO 17 & GRADE \\
\hline $6: 12$ & TAM3130 & & SBVT & A320 & DEP & DIADEMA & PERES \\
\hline $6: 14$ & GLO1293 & SJTC & & B737 & ARR & CELSO 17 & GRADE \\
\hline $6: 15$ & GLO1375 & SBDN & & B737 & ARR & CELSO 17 & GRADE \\
\hline $6: 17$ & TAM3900 & & SBRJ & A319 & DEP & DIADEMA & COSME \\
\hline $6: 19$ & GLO1500 & & SBRJ & B737 & DEP & DIADEMA & COSME \\
\hline $6: 21$ & GLO1346 & & SBVT & B737 & DEP & DIADEMA & PERES \\
\hline $6: 23$ & TAM3605 & SBSV & & A320 & ARR & CELSO 17 & PCL \\
\hline $6: 25$ & VRN2331 & SBSV & & B737 & ARR & CELSO 17 & PCL \\
\hline $6: 29$ & GLO1240 & & SBPA & B738 & DEP & APIT & CURSE \\
\hline $6: 33$ & PTN4780 & & SBJF & AT42 & DEP & DIADEMA & PERES \\
\hline $6: 35$ & TAM3763 & & SBLO & A319 & DEP & KING & SCB \\
\hline $6: 37$ & TAM3902 & & SBRJ & A319 & DEP & DIADEMA & COSME \\
\hline $6: 39$ & VRN2500 & & SBBR & B737 & DEP & DUMO & $B G C$ \\
\hline $6: 41$ & TAM3737 & SBSR & & A319 & ARR & CELSO 17 & GRADE \\
\hline $6: 43$ & TAM3242 & & SBUL & A320 & DEP & DUMO & $B G C$ \\
\hline $6: 44$ & GLO1300 & & SBNF & B737 & DEP & APIT & NIBGA \\
\hline $6: 48$ & TAM3270 & & SBRP & А320 & DEP & DUMO & $B G C$ \\
\hline $6: 49$ & TAM3035 & & SBJV & A319 & DEP & APIT & NIBGA \\
\hline $6: 50$ & TAM3471 & SBGO & & А320 & ARR & CELSO 17 & GRADE \\
\hline $6: 52$ & GLO1502 & & SBRJ & B737 & DEP & DIADEMA & COSME \\
\hline $6: 54$ & GLO1347 & SBVT & & B737 & ARR & REDE 17 & LITRE \\
\hline $6: 56$ & ONE6000 & & SBRJ & F100 & DEP & DIADEMA & COSME \\
\hline $6: 58$ & TAM3702 & & SBBR & A319 & DEP & DUMO & $B G C$ \\
\hline $6: 59$ & TAM3001 & & SBCT & А320 & DEP & APIT & NIBGA \\
\hline 7:00 & TAM3719 & SBBR & & А320 & ARR & CELSO 17 & GRADE \\
\hline 7:01 & TAM3252 & & SBGL & A320 & DEP & DIADEMA & PERES \\
\hline 7:04 & GLO1501 & SBRJ & & B737 & ARR & REDE 17 & LITRE \\
\hline 7:06 & TAM3901 & SBRJ & & A319 & ARR & REDE 17 & LITRE \\
\hline 7:08 & TAM3904 & & SBRJ & A319 & DEP & DIADEMA & COSME \\
\hline $7: 10$ & TAM3100 & SBFL & & A319 & ARR & REDE 17 & OSAMU \\
\hline $7: 12$ & VRN2151 & SBCT & & B737 & ARR & REDE 17 & PNG \\
\hline $7: 17$ & VRN2130 & & SBFL & B737 & DEP & APIT & NIBGA \\
\hline $7: 19$ & GLO1504 & & SBRJ & B737 & DEP & DIADEMA & COSME \\
\hline $7: 21$ & GLO1261 & SBCF & & B737 & ARR & CELSO 17 & PCL \\
\hline $7: 23$ & TAM3601 & SBSV & & A319 & ARR & CELSO 17 & PCL \\
\hline $7: 25$ & TAM3129 & SBVT & & A319 & ARR & REDE 17 & LITRE \\
\hline $7: 27$ & PTN4767 & SBAU & & AT42 & ARR & CELSO 17 & GRADE \\
\hline $7: 30$ & GLO1503 & SBRJ & & B737 & ARR & REDE 17 & LITRE \\
\hline
\end{tabular}




\begin{tabular}{|c|c|c|c|c|c|c|c|}
\hline Slot ATC & Voo & Origem & Destino & Eqpto & Tipo & Procedimento & Transição \\
\hline $7: 32$ & TAM3210 & & SBCF & A320 & DEP & DUMO & TEXAS \\
\hline $7: 34$ & PTN4731 & SBDN & & AT42 & ARR & CELSO 17 & GRADE \\
\hline $7: 36$ & TAM3251 & SBGL & & A320 & ARR & REDE 17 & LITRE \\
\hline $7: 38$ & GLO1260 & & SBCF & B738 & DEP & DUMO & TEXAS \\
\hline $7: 41$ & TAM3906 & & SBRJ & A319 & DEP & DIADEMA & COSME \\
\hline $7: 43$ & TAM3903 & SBRJ & & A319 & ARR & REDE 17 & LITRE \\
\hline $7: 45$ & TAM3211 & SBCF & & А320 & ARR & CELSO 17 & PCL \\
\hline $7: 46$ & ONE6001 & SBRJ & & F100 & ARR & REDE 17 & LITRE \\
\hline $7: 47$ & TAM3059 & & SBPA & A320 & DEP & APIT & CURSE \\
\hline $7: 49$ & GLO1506 & & SBRJ & B737 & DEP & DIADEMA & COSME \\
\hline $7: 50$ & ONE6150 & & SBCF & F100 & DEP & DUMO & TEXAS \\
\hline $7: 52$ & TAM3002 & SBCT & & А320 & ARR & REDE 17 & PNG \\
\hline $7: 56$ & PTN4749 & SJTC & & AT42 & ARR & CELSO 17 & GRADE \\
\hline $7: 58$ & TAM3716 & & SBBR & А320 & DEP & DUMO & $B G C$ \\
\hline $7: 59$ & GLO1200 & & SBBR & B738 & DEP & DUMO & $B G C$ \\
\hline 8:02 & TAM3740 & & SBSR & A319 & DEP & KING & SCB \\
\hline 8:04 & GLO1281 & SBFL & & B737 & ARR & REDE 17 & OSAMU \\
\hline 8:06 & PTN4762 & & SJTC & AT42 & DEP & KING & SCB \\
\hline 8:08 & GLO1505 & SBRJ & & B737 & ARR & REDE 17 & LITRE \\
\hline $8: 10$ & TAM3908 & & SBRJ & A319 & DEP & DIADEMA & COSME \\
\hline $8: 12$ & TAM3048 & SBPA & & А320 & ARR & REDE 17 & OSAMU \\
\hline $8: 14$ & TAM3905 & SBRJ & & A319 & ARR & REDE 17 & LITRE \\
\hline $8: 15$ & GLO1311 & SBUL & & B737 & ARR & CELSO 17 & GRADE \\
\hline $8: 17$ & PTN4738 & & SBML & AT42 & DEP & KING & SCB \\
\hline $8: 19$ & TAM3119 & & SBNF & A319 & DEP & APIT & NIBGA \\
\hline $8: 21$ & GLO1508 & & SBRJ & B737 & DEP & DIADEMA & COSME \\
\hline $8: 23$ & VRN2150 & & SBCT & B737 & DEP & APIT & NIBGA \\
\hline $8: 25$ & TAM3101 & & SBFL & A319 & DEP & APIT & NIBGA \\
\hline $8: 27$ & TAM3460 & & SBGO & А320 & DEP & DUMO & $B G C$ \\
\hline $8: 29$ & GLO1376 & & SBJV & B737 & DEP & APIT & NIBGA \\
\hline $8: 30$ & VRN2101 & SBPA & & B737 & ARR & REDE 17 & OSAMU \\
\hline $8: 31$ & ONE6002 & & SBRJ & F100 & DEP & DIADEMA & cosme \\
\hline $8: 33$ & VRN2051 & SBCF & & B733 & ARR & CELSO 17 & PCL \\
\hline $8: 35$ & GLO1202 & & SBBR & B737 & DEP & DUMO & $B G C$ \\
\hline $8: 37$ & GLO1507 & SBRJ & & B737 & ARR & REDE 17 & LITRE \\
\hline 8:39 & TAM3718 & & SBBR & АЗ20 & DEP & DUMO & $B G C$ \\
\hline $8: 41$ & TAM3910 & & SBRJ & A319 & DEP & DIADEMA & COSME \\
\hline $8: 44$ & PTN4782 & & SBJF & AT42 & DEP & DIADEMA & PERES \\
\hline $8: 45$ & GLO1382 & & SBGL & B738 & DEP & DIADEMA & PERES \\
\hline $8: 46$ & ONE6173 & SBFL & & F100 & ARR & REDE 17 & OSAMU \\
\hline $8: 48$ & GLO1242 & & SBPA & B737 & DEP & APIT & CURSE \\
\hline $8: 50$ & TAM3907 & SBRJ & & A319 & ARR & REDE 17 & LITRE \\
\hline $8: 52$ & GLO1510 & & SBRJ & B737 & DEP & DIADEMA & COSME \\
\hline $8: 54$ & GLO1201 & SBBR & & B737 & ARR & CELSO 17 & GRADE \\
\hline $8: 55$ & TAM3711 & SBBR & & А320 & ARR & CELSO 17 & GRADE \\
\hline
\end{tabular}




\begin{tabular}{|c|c|c|c|c|c|c|c|}
\hline Slot ATC & Voo & Origem & Destino & Eqpto & Tipo & Procedimento & Transição \\
\hline $8: 59$ & TAM3003 & & SBCT & A320 & DEP & APIT & NIBGA \\
\hline 9:02 & GLO1509 & SBRJ & & B737 & ARR & REDE 17 & LITRE \\
\hline 9:06 & TAM3216 & & SBCF & A320 & DEP & DUMO & TEXAS \\
\hline 9:08 & TAM3912 & & SBRJ & A319 & DEP & DIADEMA & COSME \\
\hline $9: 10$ & TAM3201 & SBCF & & A319 & ARR & CELSO 17 & PCL \\
\hline $9: 12$ & TAM3762 & SBLO & & A319 & ARR & CELSO 17 & GRADE \\
\hline $9: 14$ & GLO1342 & & SBCX & B737 & DEP & APIT & CURSE \\
\hline $9: 15$ & ONE6003 & SBRJ & & F100 & ARR & REDE 17 & LITRE \\
\hline $9: 17$ & TAM3909 & SBRJ & & A319 & ARR & REDE 17 & LITRE \\
\hline $9: 18$ & TAM3277 & SBRP & & А320 & ARR & CELSO 17 & GRADE \\
\hline $9: 21$ & TAM3239 & SBUL & & А320 & ARR & CELSO 17 & GRADE \\
\hline $9: 22$ & GLO1512 & & SBRJ & B737 & DEP & DIADEMA & COSME \\
\hline $9: 24$ & TAM3004 & SBCT & & A320 & ARR & REDE 17 & PNG \\
\hline $9: 26$ & GLO1289 & SBLO & & B737 & ARR & CELSO 17 & GRADE \\
\hline $9: 28$ & GLO1221 & SBCT & & B738 & ARR & REDE 17 & PNG \\
\hline $9: 30$ & VRN2152 & & SBCT & B737 & DEP & APIT & NIBGA \\
\hline 9:31 & ONE6173 & & SBBR & $\mathrm{F} 100$ & DEP & DUMO & $B G C$ \\
\hline 9:32 & PTN4781 & SBJF & & AT42 & ARR & REDE 17 & LITRE \\
\hline 9:34 & ТАМ3032 & SBJV & & A319 & ARR & REDE 17 & OSAMU \\
\hline 9:36 & VRN2050 & & SBCF & B733 & DEP & DUMO & TEXAS \\
\hline 9:38 & GLO1511 & SBRJ & & B737 & ARR & REDE 17 & LITRE \\
\hline $9: 40$ & TAM3914 & & SBRJ & A319 & DEP & DIADEMA & cosme \\
\hline 9:44 & PTN4761 & SBAU & & AT42 & ARR & CELSO 17 & GRADE \\
\hline 9:45 & GLO1263 & SBCF & & B737 & ARR & CELSO 17 & PCL \\
\hline $9: 47$ & TAM3911 & SBRJ & & A319 & ARR & REDE 17 & LITRE \\
\hline $9: 49$ & VRN2131 & SBFL & & B737 & ARR & REDE 17 & OSAMU \\
\hline $9: 51$ & GLO1514 & & SBRJ & B737 & DEP & DIADEMA & COSME \\
\hline 9:53 & GLO1241 & SBPA & & B738 & ARR & REDE 17 & OSAMU \\
\hline $9: 55$ & VRN2501 & SBBR & & B737 & ARR & CELSO 17 & GRADE \\
\hline 9:57 & GLO1280 & & SBFL & B737 & DEP & APIT & NIBGA \\
\hline 9:59 & TAM3772 & & SBCG & А320 & DEP & KING & SCB \\
\hline $10: 00$ & GLO1301 & SBNF & & B733 & ARR & REDE 17 & OSAMU \\
\hline $10: 01$ & ONE6004 & & SBRJ & F100 & DEP & DIADEMA & COSME \\
\hline $10: 02$ & GLO1265 & SBCF & & B738 & ARR & CELSO 17 & PCL \\
\hline $10: 04$ & GLO1513 & SBRJ & & B737 & ARR & REDE 17 & LITRE \\
\hline $10: 06$ & TAM3660 & & SBIL & A319 & DEP & DUMO & TEXAS \\
\hline $10: 08$ & TAM3212 & & SBCF & A319 & DEP & DUMO & TEXAS \\
\hline $10: 12$ & TAM3916 & & SBRJ & A319 & DEP & DIADEMA & cosmE \\
\hline $10: 17$ & TAM3913 & SBRJ & & A319 & ARR & REDE 17 & LITRE \\
\hline $10: 18$ & GLO1516 & & SBRJ & B737 & DEP & DIADEMA & cosme \\
\hline $10: 19$ & TAM3053 & & SBPA & А320 & DEP & APIT & CURSE \\
\hline $10: 21$ & TAM3720 & & SBBR & А320 & DEP & DUMO & $B G C$ \\
\hline $10: 23$ & PTN4712 & & SBML & AT42 & DEP & KING & SCB \\
\hline $10: 24$ & VRN2153 & SBCT & & B737 & ARR & REDE 17 & PNG \\
\hline $10: 25$ & VRN2503 & SBBR & & B737 & ARR & CELSO 17 & GRADE \\
\hline
\end{tabular}




\begin{tabular}{|c|c|c|c|c|c|c|c|}
\hline Slot ATC & Voo & Origem & Destino & Eqpto & Tipo & Procedimento & Transição \\
\hline $10: 26$ & GLO1771 & SBGO & & B737 & ARR & CELSO 17 & GRADE \\
\hline $10: 29$ & PTN4772 & SJTC & & AT42 & ARR & CELSO 17 & GRADE \\
\hline $10: 30$ & TAM3254 & & SBGL & A320 & DEP & DIADEMA & PERES \\
\hline $10: 31$ & GLO1384 & & SBGL & B738 & DEP & DIADEMA & PERES \\
\hline $10: 35$ & GLO1244 & & SBPA & B737 & DEP & APIT & CURSE \\
\hline $10: 36$ & GLO1378 & & SBJV & B737 & DEP & APIT & NIBGA \\
\hline $10: 38$ & GLO1515 & SBRJ & & B737 & ARR & REDE 17 & LITRE \\
\hline $10: 40$ & TAM3007 & & SBCT & A319 & DEP & APIT & NIBGA \\
\hline $10: 43$ & TAM3918 & & SBRJ & A319 & DEP & DIADEMA & COSME \\
\hline $10: 45$ & GLO1302 & & SBCG & B738 & DEP & KING & SCB \\
\hline $10: 47$ & TAM3213 & SBCF & & А320 & ARR & CELSO 17 & PCL \\
\hline $10: 48$ & GLO1770 & & SBGO & B737 & DEP & DUMO & $B G C$ \\
\hline $10: 50$ & GLO1518 & & SBRJ & B737 & DEP & DIADEMA & COSME \\
\hline $10: 52$ & TAM3915 & SBRJ & & A319 & ARR & REDE 17 & LITRE \\
\hline $10: 54$ & TAM3701 & SBBR & & A319 & ARR & CELSO 17 & GRADE \\
\hline $10: 56$ & GLO1377 & SBJV & & B737 & ARR & REDE 17 & OSAMU \\
\hline $10: 58$ & VRN2154 & & SBCT & B737 & DEP & APIT & NIBGA \\
\hline $11: 00$ & GLO1391 & SBGL & & B737 & ARR & REDE 17 & LITRE \\
\hline $11: 04$ & TAM3118 & SBNF & & A319 & ARR & REDE 17 & OSAMU \\
\hline $11: 06$ & GLO1290 & & SBMG & B738 & DEP & KING & SCB \\
\hline $11: 08$ & VRN2502 & & SBBR & B737 & DEP & DUMO & $B G C$ \\
\hline $11: 11$ & TAM3920 & & SBRJ & A319 & DEP & DIADEMA & cosme \\
\hline $11: 12$ & TAM3014 & SBCT & & А320 & ARR & REDE 17 & PNG \\
\hline $11: 13$ & TAM3917 & SBRJ & & A319 & ARR & REDE 17 & LITRE \\
\hline $11: 15$ & GLO1517 & SBRJ & & B737 & ARR & REDE 17 & LITRE \\
\hline $11: 17$ & VRN2330 & & SBSV & B737 & DEP & DUMO & TEXAS \\
\hline $11: 21$ & VRN2103 & SBPA & & B733 & ARR & REDE 17 & OSAMU \\
\hline $11: 23$ & GLO1520 & & SBRJ & B737 & DEP & DIADEMA & COSME \\
\hline $11: 24$ & TAM3058 & SBPA & & А320 & ARR & REDE 17 & OSAMU \\
\hline $11: 26$ & VRN2220 & & SBVT & B737 & DEP & DIADEMA & PERES \\
\hline $11: 28$ & GLO1203 & SBBR & & B738 & ARR & CELSO 17 & GRADE \\
\hline $11: 30$ & GLO1386 & & SBGL & B738 & DEP & DIADEMA & PERES \\
\hline $11: 32$ & TAM3102 & SBFL & & A319 & ARR & REDE 17 & OSAMU \\
\hline $11: 34$ & GLO1367 & SBVT & & B737 & ARR & REDE 17 & LITRE \\
\hline $11: 36$ & GLO1519 & SBRJ & & B737 & ARR & REDE 17 & LITRE \\
\hline $11: 38$ & VRN2053 & SBCF & & B733 & ARR & CELSO 17 & PCL \\
\hline $11: 40$ & GLO1310 & & SBUL & B737 & DEP & DUMO & $B G C$ \\
\hline $11: 42$ & TAM3922 & & SBRJ & A319 & DEP & DIADEMA & COSME \\
\hline $11: 44$ & TAM3138 & & SBVT & А320 & DEP & DIADEMA & PERES \\
\hline $11: 46$ & VRN2100 & & SBPA & B738 & DEP & APIT & CURSE \\
\hline $11: 48$ & GLO1522 & & SBRJ & B737 & DEP & DIADEMA & COSME \\
\hline $11: 50$ & TAM3919 & SBRJ & & A319 & ARR & REDE 17 & LITRE \\
\hline $11: 52$ & TAM3462 & & SBGO & A319 & DEP & DUMO & $B G C$ \\
\hline $11: 54$ & VRN2155 & SBCT & & B737 & ARR & REDE 17 & PNG \\
\hline $11: 55$ & VRN2052 & & SBCF & B733 & DEP & DUMO & TEXAS \\
\hline
\end{tabular}




\begin{tabular}{|c|c|c|c|c|c|c|c|}
\hline Slot ATC & Voo & Origem & Destino & Eqpto & Tipo & Procedimento & Transição \\
\hline $11: 57$ & GLO1205 & SBBR & & B737 & ARR & CELSO 17 & GRADE \\
\hline $11: 59$ & PTN4783 & SBJF & & AT42 & ARR & REDE 17 & LITRE \\
\hline $12: 00$ & TAM3039 & & SBJV & A319 & DEP & APIT & NIBGA \\
\hline $12: 02$ & PTN4763 & SJTC & & AT42 & ARR & CELSO 17 & GRADE \\
\hline $12: 04$ & GLO1521 & SBRJ & & B737 & ARR & REDE 17 & LITRE \\
\hline $12: 06$ & GLO1220 & & SBCT & B737 & DEP & APIT & NIBGA \\
\hline $12: 08$ & GLO1282 & & SBFL & B737 & DEP & APIT & NIBGA \\
\hline $12: 09$ & TAM3274 & & SBRP & А320 & DEP & DUMO & $B G C$ \\
\hline $12: 10$ & TAM3461 & SBGO & & A320 & ARR & CELSO 17 & GRADE \\
\hline $12: 12$ & TAM3924 & & SBRJ & A319 & DEP & DIADEMA & COSME \\
\hline $12: 16$ & TAM3706 & & SBBR & А320 & DEP & DUMO & $B G C$ \\
\hline $12: 18$ & ONE6007 & SBRJ & & $\mathrm{F} 100$ & ARR & REDE 17 & LITRE \\
\hline $12: 20$ & TAM3921 & SBRJ & & A319 & ARR & REDE 17 & LITRE \\
\hline $12: 22$ & GLO1524 & & SBRJ & B737 & DEP & DIADEMA & COSME \\
\hline $12: 27$ & TAM3703 & SBBR & & А320 & ARR & CELSO 17 & GRADE \\
\hline $12: 29$ & TAM3121 & & SBNF & A319 & DEP & APIT & NIBGA \\
\hline $12: 32$ & GLO1343 & SBCX & & B737 & ARR & REDE 17 & PNG \\
\hline $12: 34$ & TAM3131 & SBVT & & A319 & ARR & REDE 17 & LITRE \\
\hline $12: 35$ & GLO1523 & SBRJ & & B737 & ARR & REDE 17 & LITRE \\
\hline $12: 36$ & PTN4733 & SBML & & AT42 & ARR & CELSO 17 & GRADE \\
\hline $12: 41$ & VRN2156 & & SBCT & B733 & DEP & APIT & NIBGA \\
\hline $12: 43$ & TAM3926 & & SBRJ & A319 & DEP & DIADEMA & cosme \\
\hline $12: 44$ & PTN4784 & & SBJF & AT42 & DEP & DIADEMA & PERES \\
\hline $12: 45$ & GLO1283 & SBFL & & B737 & ARR & REDE 17 & OSAMU \\
\hline $12: 47$ & TAM3923 & SBRJ & & A319 & ARR & REDE 17 & LITRE \\
\hline $12: 49$ & GLO1526 & & SBRJ & B737 & DEP & DIADEMA & COSME \\
\hline $12: 51$ & GLO1385 & SBGL & & B738 & ARR & REDE 17 & LITRE \\
\hline $12: 53$ & TAM3008 & SBCT & & A319 & ARR & REDE 17 & PNG \\
\hline $12: 55$ & VRN2504 & & SBBR & B737 & DEP & DUMO & $B G C$ \\
\hline $12: 57$ & TAM3215 & SBCF & & A319 & ARR & CELSO 17 & PCL \\
\hline $13: 01$ & GLO1379 & SBJV & & B737 & ARR & REDE 17 & OSAMU \\
\hline 13:04 & TAM3132 & & SBVT & А320 & DEP & DIADEMA & PERES \\
\hline 13:05 & ONE6008 & & SBRJ & F100 & DEP & DIADEMA & COSME \\
\hline 13:06 & TAM3925 & SBRJ & & A319 & ARR & REDE 17 & LITRE \\
\hline $13: 08$ & TAM3771 & SBCG & & A320 & ARR & CELSO 17 & GRADE \\
\hline $13: 10$ & GLO1525 & SBRJ & & B737 & ARR & REDE 17 & LITRE \\
\hline $13: 13$ & TAM3928 & & SBRJ & A319 & DEP & DIADEMA & COSME \\
\hline $13: 16$ & GLO1291 & SBMG & & B738 & ARR & CELSO 17 & GRADE \\
\hline $13: 18$ & GLO1360 & & SBNF & B737 & DEP & APIT & NIBGA \\
\hline $13: 20$ & GLO1397 & SBSV & & B737 & ARR & CELSO 17 & PCL \\
\hline $13: 21$ & GLO1528 & & SBRJ & B737 & DEP & DIADEMA & COSME \\
\hline $13: 24$ & GLO1204 & & SBBR & B737 & DEP & DUMO & $B G C$ \\
\hline $13: 27$ & GLO1207 & SBBR & & B737 & ARR & CELSO 17 & GRADE \\
\hline $13: 28$ & GLO1395 & & SBGL & B738 & DEP & DIADEMA & PERES \\
\hline $13: 29$ & TAM3061 & & SBPA & А320 & DEP & APIT & CURSE \\
\hline
\end{tabular}




\begin{tabular}{|c|c|c|c|c|c|c|c|}
\hline Slot ATC & Voo & Origem & Destino & Eqpto & Tipo & Procedimento & Transição \\
\hline $13: 30$ & PTN4716 & & SBML & AT42 & DEP & KING & SCB \\
\hline $13: 32$ & TAM3009 & & $\mathrm{SBCT}$ & A319 & DEP & APIT & NIBGA \\
\hline $13: 33$ & GLO1527 & SBRJ & & B737 & ARR & REDE 17 & LITRE \\
\hline $13: 35$ & TAM3927 & SBRJ & & A319 & ARR & REDE 17 & LITRE \\
\hline $13: 37$ & TAM3930 & & SBRJ & A319 & DEP & DIADEMA & COSME \\
\hline $13: 39$ & GLO1358 & & SBDN & B737 & DEP & KING & SCB \\
\hline $13: 42$ & GLO1303 & SBCG & & B738 & ARR & CELSO 17 & GRADE \\
\hline $13: 45$ & TAM3052 & SBPA & & А320 & ARR & REDE 17 & OSAMU \\
\hline $13: 46$ & GLO1243 & SBPA & & B737 & ARR & REDE 17 & OSAMU \\
\hline $13: 47$ & TAM3244 & & SBUL & A319 & DEP & DUMO & $B G C$ \\
\hline $13: 50$ & GLO1530 & & SBRJ & B737 & DEP & DIADEMA & cosme \\
\hline $13: 52$ & TAM3708 & & SBBR & A319 & DEP & DUMO & $B G C$ \\
\hline $13: 54$ & GLO1262 & & SBCF & B738 & DEP & DUMO & TEXAS \\
\hline $13: 56$ & GLO1371 & SBCY & & B738 & ARR & CELSO 17 & GRADE \\
\hline $13: 59$ & TAM3220 & & SBCF & А320 & DEP & DUMO & TEXAS \\
\hline $14: 00$ & GL01396 & & SBSV & B737 & DEP & DUMO & TEXAS \\
\hline $14: 02$ & GLO1529 & SBRJ & & B737 & ARR & REDE 17 & LITRE \\
\hline $14: 04$ & GLO1350 & & SBGO & B737 & DEP & DUMO & $B G C$ \\
\hline $14: 07$ & TAM3929 & SBRJ & & A319 & ARR & REDE 17 & LITRE \\
\hline $14: 08$ & TAM3932 & & SBRJ & A319 & DEP & DIADEMA & COSME \\
\hline $14: 10$ & GLO1773 & SBGO & & B737 & ARR & CELSO 17 & GRADE \\
\hline $14: 12$ & GLO1366 & & SBVT & B737 & DEP & DIADEMA & PERES \\
\hline $14: 18$ & GLO1532 & & SBRJ & B737 & DEP & DIADEMA & COSME \\
\hline $14: 20$ & VRN2055 & SBCF & & B733 & ARR & CELSO 17 & PCL \\
\hline $14: 22$ & GLO1223 & SBCT & & B737 & ARR & REDE 17 & PNG \\
\hline $14: 24$ & GLO1389 & & SBGL & B737 & DEP & DIADEMA & PERES \\
\hline $14: 25$ & TAM3271 & SBRP & & А320 & ARR & CELSO 17 & GRADE \\
\hline $14: 26$ & GLO1313 & SBUL & & B737 & ARR & CELSO 17 & GRADE \\
\hline $14: 28$ & TAM3038 & SBJV & & A319 & ARR & REDE 17 & OSAMU \\
\hline $14: 29$ & GLO1531 & SBRJ & & B737 & ARR & REDE 17 & LITRE \\
\hline $14: 32$ & TAM3931 & SBRJ & & A319 & ARR & REDE 17 & LITRE \\
\hline $14: 36$ & PTN4717 & SBML & & AT42 & ARR & CELSO 17 & GRADE \\
\hline $14: 38$ & TAM3934 & & SBRJ & A319 & DEP & DIADEMA & COSME \\
\hline $14: 40$ & TAM3709 & SBBR & & А320 & ARR & CELSO 17 & GRADE \\
\hline $14: 42$ & VRN2105 & SBPA & & B738 & ARR & REDE 17 & OSAMU \\
\hline $14: 43$ & TAM3011 & & SBCT & A319 & DEP & APIT & NIBGA \\
\hline $14: 44$ & VRN2221 & SBVT & & B737 & ARR & REDE 17 & LITRE \\
\hline $14: 46$ & GLO1390 & SBGL & & B737 & ARR & REDE 17 & LITRE \\
\hline $14: 47$ & TAM3010 & SBCT & & А320 & ARR & REDE 17 & PNG \\
\hline $14: 48$ & GLO1222 & & SBCT & B738 & DEP & APIT & NIBGA \\
\hline $14: 49$ & VRN2157 & SBCT & & B733 & ARR & REDE 17 & PNG \\
\hline $14: 51$ & GLO1534 & & SBRJ & B737 & DEP & DIADEMA & COSME \\
\hline $14: 52$ & TAM3745 & SBSR & & A319 & ARR & CELSO 17 & GRADE \\
\hline $14: 54$ & VRN2102 & & SBPA & B737 & DEP & APIT & CURSE \\
\hline $14: 56$ & GLO1533 & SBRJ & & B737 & ARR & REDE 17 & LITRE \\
\hline
\end{tabular}




\begin{tabular}{|c|c|c|c|c|c|c|c|}
\hline Slot ATC & Voo & Origem & Destino & Eqpto & Tipo & Procedimento & Transição \\
\hline $14: 58$ & TAM3120 & SBNF & & A319 & ARR & REDE 17 & OSAMU \\
\hline $15: 00$ & GLO1370 & & SBCY & B738 & DEP & KING & SCB \\
\hline $15: 02$ & GLO1206 & & SBBR & B738 & DEP & DUMO & $B G C$ \\
\hline $15: 04$ & TAM3463 & SBGO & & A319 & ARR & CELSO 17 & GRADE \\
\hline $15: 05$ & VRN2505 & SBBR & & B737 & ARR & CELSO 17 & GRADE \\
\hline $15: 06$ & TAM3203 & SBCF & & A320 & ARR & CELSO 17 & PCL \\
\hline $15: 08$ & TAM3933 & SBRJ & & A319 & ARR & REDE 17 & LITRE \\
\hline $15: 12$ & TAM3936 & & SBRJ & A319 & DEP & DIADEMA & COSME \\
\hline $15: 15$ & VRN2054 & & SBCF & B733 & DEP & DUMO & TEXAS \\
\hline $15: 18$ & GLO1536 & & SBRJ & B737 & DEP & DIADEMA & COSME \\
\hline $15: 22$ & TAM3222 & & SBCF & A320 & DEP & DUMO & TEXAS \\
\hline $15: 24$ & GLO1353 & SBGO & & B737 & ARR & CELSO 17 & GRADE \\
\hline $15: 25$ & ONE6009 & SBRJ & & $\mathrm{F} 100$ & ARR & REDE 17 & LITRE \\
\hline $15: 26$ & TAM3107 & & SBFL & A319 & DEP & APIT & NIBGA \\
\hline $15: 28$ & GLO1535 & SBRJ & & B737 & ARR & REDE 17 & LITRE \\
\hline $15: 29$ & VRN2158 & & $\mathrm{SBCT}$ & B733 & DEP & APIT & NIBGA \\
\hline $15: 32$ & PTN4770 & & SJTC & AT42 & DEP & KING & SCB \\
\hline $15: 33$ & TAM3935 & SBRJ & & A319 & ARR & REDE 17 & LITRE \\
\hline $15: 35$ & TAM3055 & & SBPA & А320 & DEP & APIT & CURSE \\
\hline $15: 37$ & TAM3938 & & SBRJ & A319 & DEP & DIADEMA & COSME \\
\hline $15: 39$ & GL01538 & & SBRJ & B737 & DEP & DIADEMA & COSME \\
\hline $15: 41$ & TAM3770 & & SBCG & А320 & DEP & KING & SCB \\
\hline $15: 46$ & TAM3013 & & SBCT & A319 & DEP & APIT & NIBGA \\
\hline $15: 49$ & GLO1267 & SBCF & & B737 & ARR & CELSO 17 & PCL \\
\hline $15: 53$ & TAM3744 & & SBSR & A319 & DEP & KING & SCB \\
\hline $15: 54$ & VRN2107 & SBPA & & B738 & ARR & REDE 17 & OSAMU \\
\hline $15: 56$ & TAM3202 & & SBCF & А320 & DEP & DUMO & TEXAS \\
\hline $15: 58$ & TAM3710 & & SBBR & A319 & DEP & DUMO & $B G C$ \\
\hline $16: 02$ & TAM3937 & SBRJ & & A319 & ARR & REDE 17 & LITRE \\
\hline $16: 04$ & GLO1537 & SBRJ & & B737 & ARR & REDE 17 & LITRE \\
\hline $16: 07$ & TAM3940 & & SBRJ & A319 & DEP & DIADEMA & COSME \\
\hline $16: 09$ & VRN2159 & SBCT & & B737 & ARR & REDE 17 & PNG \\
\hline $16: 11$ & GLO1392 & & SBGL & B737 & DEP & DIADEMA & PERES \\
\hline $16: 13$ & GLO1540 & & SBRJ & B737 & DEP & DIADEMA & COSME \\
\hline $16: 14$ & TAM3060 & SBPA & & A320 & ARR & REDE 17 & OSAMU \\
\hline $16: 16$ & PTN4785 & SBJF & & AT42 & ARR & REDE 17 & LITRE \\
\hline $16: 18$ & GLO1344 & & SBCX & B737 & DEP & APIT & CURSE \\
\hline $16: 20$ & VRN2132 & & SBFL & B737 & DEP & APIT & NIBGA \\
\hline $16: 22$ & VRN2333 & SBSV & & B737 & ARR & CELSO 17 & PCL \\
\hline $16: 24$ & TAM3133 & SBVT & & А320 & ARR & REDE 17 & LITRE \\
\hline $16: 25$ & VRN2056 & & SBCF & B738 & DEP & DUMO & TEXAS \\
\hline $16: 27$ & TAM3241 & SBUL & & A319 & ARR & CELSO 17 & GRADE \\
\hline $16: 29$ & VRN2506 & & SBBR & B737 & DEP & DUMO & $B G C$ \\
\hline $16: 30$ & GLO1224 & & SBCT & B737 & DEP & APIT & NIBGA \\
\hline $16: 31$ & VRN2104 & & SBPA & B738 & DEP & APIT & CURSE \\
\hline
\end{tabular}




\begin{tabular}{|c|c|c|c|c|c|c|c|}
\hline Slot ATC & Voo & Origem & Destino & Eqpto & Tipo & Procedimento & Transição \\
\hline $16: 33$ & GLO1539 & SBRJ & & B737 & ARR & REDE 17 & LITRE \\
\hline $16: 35$ & TAM3939 & SBRJ & & A319 & ARR & REDE 17 & LITRE \\
\hline $16: 37$ & TAM3942 & & SBRJ & A319 & DEP & DIADEMA & COSME \\
\hline $16: 39$ & GLO1359 & SBDN & & B737 & ARR & CELSO 17 & GRADE \\
\hline $16: 42$ & TAM3257 & SBGL & & A320 & ARR & REDE 17 & LITRE \\
\hline $16: 44$ & GLO1361 & SBNF & & B737 & ARR & REDE 17 & OSAMU \\
\hline $16: 45$ & GLO1354 & & SBGO & B737 & DEP & DUMO & $B G C$ \\
\hline $16: 48$ & GLO1542 & & SBRJ & B737 & DEP & DIADEMA & COSME \\
\hline $16: 50$ & VRN2507 & SBBR & & B737 & ARR & CELSO 17 & GRADE \\
\hline $16: 54$ & GLO1541 & SBRJ & & B737 & ARR & REDE 17 & LITRE \\
\hline $16: 56$ & TAM3000 & SBCT & & A319 & ARR & REDE 17 & PNG \\
\hline $16: 57$ & ONE6011 & SBRJ & & $\mathrm{F} 100$ & ARR & REDE 17 & LITRE \\
\hline $16: 59$ & VRN2057 & SBCF & & B738 & ARR & CELSO 17 & PCL \\
\hline $17: 01$ & TAM3223 & SBCF & & А320 & ARR & CELSO 17 & PCL \\
\hline $17: 03$ & PTN4764 & & SJTC & AT42 & DEP & KING & SCB \\
\hline $17: 05$ & TAM3722 & & SBBR & A320 & DEP & DUMO & $B G C$ \\
\hline $17: 07$ & TAM3944 & & SBRJ & A319 & DEP & DIADEMA & COSME \\
\hline 17:09 & GLO1994 & & SBNF & B737 & DEP & APIT & NIBGA \\
\hline $17: 10$ & PTN4769 & SJTC & & AT42 & ARR & CELSO 17 & GRADE \\
\hline $17: 12$ & TAM3941 & SBRJ & & A319 & ARR & REDE 17 & LITRE \\
\hline $17: 14$ & TAM3661 & SBIL & & A319 & ARR & CELSO 17 & PCL \\
\hline $17: 16$ & GLO1380 & & SBJV & B737 & DEP & APIT & NIBGA \\
\hline $17: 18$ & TAM3134 & & SBVT & А320 & DEP & DIADEMA & PERES \\
\hline $17: 20$ & TAM3123 & & SBNF & A319 & DEP & APIT & NIBGA \\
\hline $17: 22$ & GLO1544 & & SBRJ & B737 & DEP & DIADEMA & COSME \\
\hline $17: 24$ & GLO1369 & SBVT & & B737 & ARR & REDE 17 & LITRE \\
\hline $17: 26$ & GLO1543 & SBRJ & & B737 & ARR & REDE 17 & LITRE \\
\hline $17: 28$ & GLO1246 & & SBPA & B737 & DEP & APIT & CURSE \\
\hline $17: 29$ & GLO1209 & SBBR & & B737 & ARR & CELSO 17 & GRADE \\
\hline $17: 30$ & GLO1264 & & SBCF & B737 & DEP & DUMO & TEXAS \\
\hline $17: 32$ & GLO1285 & SBFL & & B737 & ARR & REDE 17 & OSAMU \\
\hline $17: 35$ & TAM3943 & SBRJ & & A319 & ARR & REDE 17 & LITRE \\
\hline $17: 37$ & TAM3946 & & SBRJ & A319 & DEP & DIADEMA & COSME \\
\hline $17: 39$ & TAM3057 & & SBPA & А320 & DEP & APIT & CURSE \\
\hline $17: 40$ & ONE6012 & & SBRJ & $\mathrm{F} 100$ & DEP & DIADEMA & COSME \\
\hline $17: 41$ & TAM3723 & SBBR & & A319 & ARR & CELSO 17 & GRADE \\
\hline $17: 43$ & GLO1546 & & SBRJ & B737 & DEP & DIADEMA & COSME \\
\hline $17: 48$ & GLO1394 & SBGL & & B738 & ARR & REDE 17 & LITRE \\
\hline $17: 52$ & TAM3015 & & SBCT & A319 & DEP & APIT & NIBGA \\
\hline $17: 54$ & TAM3012 & SBCT & & A319 & ARR & REDE 17 & PNG \\
\hline $17: 56$ & GLO1545 & SBRJ & & B737 & ARR & REDE 17 & LITRE \\
\hline $17: 58$ & TAM3468 & & SBGO & A320 & DEP & DUMO & $B G C$ \\
\hline $17: 59$ & TAM3104 & SBFL & & A319 & ARR & REDE 17 & OSAMU \\
\hline $18: 00$ & PTN4732 & & SBML & AT42 & DEP & KING & SCB \\
\hline $18: 02$ & VRN2059 & SBCF & & B733 & ARR & CELSO 17 & PCL \\
\hline
\end{tabular}




\begin{tabular}{|c|c|c|c|c|c|c|c|}
\hline Slot ATC & Voo & Origem & Destino & Eqpto & Tipo & Procedimento & Transição \\
\hline $18: 04$ & TAM3945 & SBRJ & & A319 & ARR & REDE 17 & LITRE \\
\hline 18:06 & TAM3109 & & SBFL & A319 & DEP & APIT & NIBGA \\
\hline $18: 07$ & TAM3225 & SBCF & & A320 & ARR & CELSO 17 & PCL \\
\hline $18: 08$ & TAM3948 & & SBRJ & A319 & DEP & DIADEMA & COSME \\
\hline $18: 10$ & GLO1398 & & SBSV & B737 & DEP & DUMO & TEXAS \\
\hline $18: 12$ & VRN2134 & & SBFL & B737 & DEP & APIT & NIBGA \\
\hline $18: 17$ & VRN2161 & SBCT & & B733 & ARR & REDE 17 & PNG \\
\hline $18: 19$ & TAM3743 & SBSR & & A319 & ARR & CELSO 17 & GRADE \\
\hline $18: 20$ & GLO1548 & & SBRJ & B737 & DEP & DIADEMA & cosme \\
\hline $18: 21$ & ONE6013 & SBRJ & & F100 & ARR & REDE 17 & LITRE \\
\hline $18: 22$ & VRN2109 & SBPA & & B737 & ARR & REDE 17 & OSAMU \\
\hline $18: 24$ & GLO1547 & SBRJ & & B737 & ARR & REDE 17 & LITRE \\
\hline $18: 26$ & TAM3137 & SBVT & & A320 & ARR & REDE 17 & LITRE \\
\hline $18: 28$ & GLO1208 & & SBBR & B737 & DEP & DUMO & $B G C$ \\
\hline $18: 30$ & PTN4788 & & SBJF & AT42 & DEP & DIADEMA & PERES \\
\hline $18: 31$ & TAM3767 & & SBLO & A319 & DEP & KING & SCB \\
\hline $18: 33$ & GLO1226 & & SBCT & B738 & DEP & APIT & NIBGA \\
\hline $18: 35$ & TAM3947 & SBRJ & & A319 & ARR & REDE 17 & LITRE \\
\hline $18: 37$ & TAM3950 & & SBRJ & A319 & DEP & DIADEMA & COSME \\
\hline $18: 40$ & GLO1355 & SBGO & & B737 & ARR & CELSO 17 & GRADE \\
\hline $18: 42$ & GLO1312 & & SBUL & B738 & DEP & DUMO & $B G C$ \\
\hline $18: 44$ & VRN2058 & & SBCF & B738 & DEP & DUMO & TEXAS \\
\hline $18: 45$ & GLO1550 & & SBRJ & B737 & DEP & DIADEMA & COSME \\
\hline $18: 47$ & TAM3224 & & SBCF & A319 & DEP & DUMO & TEXAS \\
\hline $18: 50$ & TAM3046 & SBPA & & A320 & ARR & REDE 17 & OSAMU \\
\hline $18: 52$ & TAM3717 & SBBR & & А320 & ARR & CELSO 17 & GRADE \\
\hline $18: 56$ & TAM3033 & & SBJV & A319 & DEP & APIT & NIBGA \\
\hline $18: 58$ & GLO1549 & SBRJ & & B737 & ARR & REDE 17 & LITRE \\
\hline 19:00 & TAM3021 & & SBCT & A320 & DEP & APIT & NIBGA \\
\hline $19: 01$ & VRN2106 & & SBPA & B733 & DEP & APIT & CURSE \\
\hline $19: 03$ & TAM3773 & SBCG & & A320 & ARR & CELSO 17 & GRADE \\
\hline $19: 05$ & GLO1772 & & SBGO & B737 & DEP & DUMO & $B G C$ \\
\hline $19: 07$ & TAM3952 & & SBRJ & A319 & DEP & DIADEMA & COSME \\
\hline 19:09 & TAM3949 & SBRJ & & A319 & ARR & REDE 17 & LITRE \\
\hline $19: 10$ & ONE6014 & & SBRJ & F100 & DEP & DIADEMA & COSME \\
\hline $19: 12$ & GLO1552 & & SBRJ & B737 & DEP & DIADEMA & cosme \\
\hline $19: 15$ & TAM3238 & & SBUL & A319 & DEP & DUMO & $B G C$ \\
\hline $19: 19$ & VRN2061 & SBCF & & B738 & ARR & CELSO 17 & PCL \\
\hline $19: 21$ & GLO1210 & & SBBR & B738 & DEP & DUMO & $B G C$ \\
\hline $19: 23$ & TAM3712 & & SBBR & А320 & DEP & DUMO & $B G C$ \\
\hline $19: 25$ & GLO1381 & SBJV & & B737 & ARR & REDE 17 & OSAMU \\
\hline $19: 27$ & GLO1551 & SBRJ & & B737 & ARR & REDE 17 & LITRE \\
\hline $19: 29$ & TAM3721 & SBBR & & A320 & ARR & CELSO 17 & GRADE \\
\hline $19: 30$ & PTN4771 & SJTC & & AT42 & ARR & CELSO 17 & GRADE \\
\hline $19: 32$ & GLO1399 & SBSV & & B737 & ARR & CELSO 17 & PCL \\
\hline
\end{tabular}




\begin{tabular}{|c|c|c|c|c|c|c|c|}
\hline Slot ATC & Voo & Origem & Destino & Eqpto & Tipo & Procedimento & Transição \\
\hline 19:34 & GLO1211 & SBBR & & B737 & ARR & CELSO 17 & GRADE \\
\hline $19: 36$ & TAM3951 & SBRJ & & A319 & ARR & REDE 17 & LITRE \\
\hline 19:38 & TAM3954 & & SBRJ & A319 & DEP & DIADEMA & COSME \\
\hline $19: 40$ & GLO1388 & & SBGL & B737 & DEP & DIADEMA & PERES \\
\hline $19: 44$ & GLO1393 & SBGL & & B738 & ARR & REDE 17 & LITRE \\
\hline $19: 45$ & GLO1225 & SBCT & & B738 & ARR & REDE 17 & PNG \\
\hline $19: 47$ & TAM3276 & & SBRP & А320 & DEP & DUMO & $B G C$ \\
\hline $19: 49$ & TAM3122 & SBNF & & A319 & ARR & REDE 17 & OSAMU \\
\hline $19: 51$ & PTN4773 & SJTC & & AT42 & ARR & CELSO 17 & GRADE \\
\hline $19: 52$ & TAM3724 & & SBBR & А320 & DEP & DUMO & $B G C$ \\
\hline $19: 54$ & GLO1554 & & SBRJ & B737 & DEP & DIADEMA & cosme \\
\hline $19: 55$ & VRN2111 & SBPA & & B738 & ARR & REDE 17 & OSAMU \\
\hline $19: 57$ & TAM3727 & SBBR & & A319 & ARR & CELSO 17 & GRADE \\
\hline $19: 59$ & TAM3250 & & SBGL & A320 & DEP & DIADEMA & PERES \\
\hline $20: 00$ & TAM3016 & SBCT & & A319 & ARR & REDE 17 & PNG \\
\hline 20:01 & ONE6015 & SBRJ & & $\mathrm{F} 100$ & ARR & REDE 17 & LITRE \\
\hline $20: 02$ & GLO1995 & SBNF & & B737 & ARR & REDE 17 & OSAMU \\
\hline 20:05 & GLO1553 & SBRJ & & B737 & ARR & REDE 17 & LITRE \\
\hline 20:07 & TAM3956 & & SBRJ & A319 & DEP & DIADEMA & COSME \\
\hline $20: 10$ & VRN2060 & & SBCF & B738 & DEP & DUMO & TEXAS \\
\hline $20: 11$ & GLO1345 & SBCX & & B737 & ARR & REDE 17 & PNG \\
\hline $20: 12$ & TAM3953 & SBRJ & & A319 & ARR & REDE 17 & LITRE \\
\hline $20: 14$ & PTN4734 & & SBDN & AT42 & DEP & KING & SCB \\
\hline $20: 15$ & GLO1212 & & SBBR & B737 & DEP & DUMO & $B G C$ \\
\hline $20: 16$ & GLO1288 & & SBLO & B737 & DEP & KING & SCB \\
\hline 20:20 & GLO1556 & & SBRJ & B737 & DEP & DIADEMA & COSME \\
\hline $20: 22$ & TAM3217 & SBCF & & А320 & ARR & CELSO 17 & PCL \\
\hline $20: 24$ & GLO1213 & SBBR & & B738 & ARR & CELSO 17 & GRADE \\
\hline 20:25 & TAM3725 & SBBR & & А320 & ARR & CELSO 17 & GRADE \\
\hline $20: 27$ & TAM3604 & & SBSV & А320 & DEP & DUMO & TEXAS \\
\hline $20: 29$ & TAM3259 & SBGL & & А320 & ARR & REDE 17 & LITRE \\
\hline $20: 32$ & TAM3955 & SBRJ & & A319 & ARR & REDE 17 & LITRE \\
\hline $20: 34$ & TAM3226 & & SBCF & A319 & DEP & DUMO & TEXAS \\
\hline $20: 36$ & GLO1555 & SBRJ & & B737 & ARR & REDE 17 & LITRE \\
\hline $20: 40$ & VRN2062 & & SBCF & B733 & DEP & DUMO & TEXAS \\
\hline $20: 42$ & TAM3135 & SBVT & & А320 & ARR & REDE 17 & LITRE \\
\hline $20: 44$ & VRN2160 & & SBCT & B737 & DEP & APIT & NIBGA \\
\hline $20: 45$ & ONE6016 & & SBRJ & $\mathrm{F} 100$ & DEP & DIADEMA & cosmE \\
\hline $20: 48$ & TAM3105 & & SBFL & A319 & DEP & APIT & NIBGA \\
\hline $20: 50$ & GLO1558 & & SBRJ & B737 & DEP & DIADEMA & cosme \\
\hline $20: 52$ & VRN2108 & & SBPA & B738 & DEP & APIT & CURSE \\
\hline $20: 54$ & VRN2133 & SBFL & & B737 & ARR & REDE 17 & OSAMU \\
\hline $20: 55$ & ONE6172 & SBBR & & $\mathrm{F} 100$ & ARR & CELSO 17 & GRADE \\
\hline $20: 57$ & GLO1557 & SBRJ & & B737 & ARR & REDE 17 & LITRE \\
\hline $20: 59$ & TAM3110 & SBFL & & A319 & ARR & REDE 17 & OSAMU \\
\hline
\end{tabular}




\begin{tabular}{|c|c|c|c|c|c|c|c|}
\hline Slot ATC & Voo & Origem & Destino & Eqpto & Tipo & Procedimento & Transição \\
\hline $21: 01$ & TAM3715 & SBBR & & A320 & ARR & CELSO 17 & GRADE \\
\hline 21:02 & TAM3128 & & SBVT & A319 & DEP & DIADEMA & PERES \\
\hline 21:03 & TAM3800 & & SBCY & A320 & DEP & KING & SCB \\
\hline $21: 07$ & TAM3764 & SBLO & & A319 & ARR & CELSO 17 & GRADE \\
\hline $21: 09$ & TAM3958 & & SBRJ & A319 & DEP & DIADEMA & COSME \\
\hline $21: 11$ & TAM3957 & SBRJ & & A319 & ARR & REDE 17 & LITRE \\
\hline $21: 12$ & TAM3020 & SBCT & & А320 & ARR & REDE 17 & PNG \\
\hline $21: 13$ & TAM3714 & & SBBR & А320 & DEP & DUMO & $B G C$ \\
\hline $21: 14$ & PTN4747 & SJTC & & AT42 & ARR & CELSO 17 & GRADE \\
\hline $21: 16$ & TAM3034 & SBJV & & A319 & ARR & REDE 17 & OSAMU \\
\hline $21: 18$ & TAM3228 & & SBCF & А320 & DEP & DUMO & TEXAS \\
\hline $21: 22$ & TAM3019 & & SBCT & А320 & DEP & APIT & NIBGA \\
\hline $21: 24$ & PTN4789 & SBJF & & AT42 & ARR & REDE 17 & LITRE \\
\hline $21: 25$ & GLO1560 & & SBRJ & B737 & DEP & DIADEMA & COSME \\
\hline $21: 27$ & TAM3469 & SBGO & & А320 & ARR & CELSO 17 & GRADE \\
\hline $21: 29$ & TAM3056 & SBPA & & А320 & ARR & REDE 17 & OSAMU \\
\hline $21: 30$ & ONE6017 & SBRJ & & $\mathrm{F} 100$ & ARR & REDE 17 & LITRE \\
\hline $21: 31$ & GLO1214 & & SBBR & B738 & DEP & DUMO & $B G C$ \\
\hline $21: 35$ & GLO1559 & SBRJ & & B737 & ARR & REDE 17 & LITRE \\
\hline $21: 38$ & TAM3470 & & SBGO & A320 & DEP & DUMO & $B G C$ \\
\hline $21: 40$ & TAM3959 & SBRJ & & A319 & ARR & REDE 17 & LITRE \\
\hline $21: 42$ & GLO1266 & & SBCF & B737 & DEP & DUMO & TEXAS \\
\hline $21: 44$ & GLO1245 & SBPA & & B737 & ARR & REDE 17 & OSAMU \\
\hline $21: 45$ & ONE6172 & & SBFL & $\mathrm{F} 100$ & DEP & APIT & NIBGA \\
\hline $21: 47$ & GLO1227 & SBCT & & B738 & ARR & REDE 17 & PNG \\
\hline $21: 49$ & PTN4719 & SBML & & AT42 & ARR & CELSO 17 & GRADE \\
\hline $21: 50$ & TAM3600 & & SBSV & A319 & DEP & DUMO & TEXAS \\
\hline $21: 52$ & TAM3227 & SBCF & & A319 & ARR & CELSO 17 & PCL \\
\hline $21: 54$ & TAM3245 & SBUL & & A319 & ARR & CELSO 17 & GRADE \\
\hline $21: 55$ & GLO1273 & SBCF & & B738 & ARR & CELSO 17 & PCL \\
\hline $21: 57$ & PTN4760 & & SBAU & AT42 & DEP & KING & SCB \\
\hline $21: 58$ & TAM3736 & & SBSR & A319 & DEP & KING & SCB \\
\hline $22: 00$ & GLO1562 & & SBRJ & B737 & DEP & DIADEMA & cosme \\
\hline 22:01 & GLO1561 & SBRJ & & B737 & ARR & REDE 17 & LITRE \\
\hline $22: 03$ & TAM3275 & SBRP & & А320 & ARR & CELSO 17 & GRADE \\
\hline 22:05 & TAM3049 & & SBPA & А320 & DEP & APIT & CURSE \\
\hline $22: 07$ & TAM3960 & & SBRJ & A319 & DEP & DIADEMA & cosme \\
\hline $22: 09$ & TAM3774 & & SBCG & A319 & DEP & KING & SCB \\
\hline $22: 10$ & PTN4748 & & SJTC & AT42 & DEP & KING & SCB \\
\hline $22: 12$ & GLO1215 & SBBR & & B737 & ARR & CELSO 17 & GRADE \\
\hline $22: 14$ & TAM3961 & SBRJ & & A319 & ARR & REDE 17 & LITRE \\
\hline $22: 15$ & GLO1292 & & SJTC & B737 & DEP & KING & SCB \\
\hline $22: 17$ & VRN2135 & SBFL & & B737 & ARR & REDE 17 & OSAMU \\
\hline $22: 19$ & GLO1374 & & SBDN & B737 & DEP & KING & SCB \\
\hline $22: 20$ & PTN4766 & & SBAU & AT42 & DEP & KING & SCB \\
\hline
\end{tabular}




\begin{tabular}{cccccccc}
\hline Slot ATC & Voo & Origem & Destino & Eqpto & Tipo & Procedimento & Transição \\
\hline $22: 22$ & VRN2332 & & SBSV & B737 & DEP & DUMO & TEXAS \\
22:24 & GLO1368 & & SBVT & B737 & DEP & DIADEMA & PERES \\
22:25 & ONE6151 & SBCF & & F100 & ARR & CELSO 17 & PCL \\
22:27 & GLO1286 & & SBFL & B737 & DEP & APIT & NIBGA \\
\hline
\end{tabular}


C. 3 - Voos excluídos para redução de $5 \%$ no volume de tráfego

\begin{tabular}{cccccccc}
\hline Slot ATC & Voo & Origem & Destino & Eqpto & Tipo & Procedimento & Transição \\
\hline 7:06 & TAM3901 & SBRJ & & A319 & ARR & REDE 17 & LITRE \\
8:15 & GLO1311 & SBUL & & B737 & ARR & CELSO 17 & GRADE \\
8:55 & TAM3711 & SBBR & & A320 & ARR & CELSO 17 & GRADE \\
9:17 & TAM3909 & SBRJ & & A319 & ARR & REDE 17 & LITRE \\
10:21 & TAM3720 & & SBBR & A320 & DEP & DUMO & BGC \\
11:36 & GLO1519 & SBRJ & & B737 & ARR & REDE 17 & LITRE \\
12:20 & TAM3921 & SBRJ & & A319 & ARR & REDE 17 & LITRE \\
13:05 & ONE6008 & & SBRJ & F100 & DEP & DIADEMA & COSME \\
13:08 & TAM3771 & SBCG & & A320 & ARR & CELSO 17 & GRADE \\
14:28 & TAM3038 & SBJV & & A319 & ARR & REDE 17 & OSAMU \\
14:48 & GLO1222 & & SBCT & B738 & DEP & APIT & NIBGA \\
16:22 & VRN2333 & SBSV & & B737 & ARR & CELSO 17 & PCL \\
16:31 & VRN2104 & & SBPA & B738 & DEP & APIT & CURSE \\
17:10 & PTN4769 & SJTC & & AT42 & ARR & CELSO 17 & GRADE \\
17:16 & GLO1380 & & SBJV & B737 & DEP & APIT & NIBGA \\
17:32 & GLO1285 & SBFL & & B737 & ARR & REDE 17 & OSAMU \\
18:02 & VRN2059 & SBCF & & B733 & ARR & CELSO 17 & PCL \\
18:42 & GLO1312 & & SBUL & B738 & DEP & DUMO & BGC \\
19:05 & GLO1772 & & SBGO & B737 & DEP & DUMO & BGC \\
19:29 & TAM3721 & SBBR & & A320 & ARR & CELSO 17 & GRADE \\
19:57 & TAM3727 & SBBR & & A319 & ARR & CELSO 17 & GRADE \\
20:11 & GLO1345 & SBCX & & B737 & ARR & REDE 17 & PNG \\
20:16 & GLO1288 & & SBLO & B737 & DEP & KING & SCB \\
21:11 & TAM3957 & SBRJ & & A319 & ARR & REDE 17 & LITRE \\
21:22 & TAM3019 & & SBCT & A320 & DEP & APIT & NIBGA \\
\hline
\end{tabular}


C.4 - Voos excluídos para redução de $10 \%$ no volume de tráfego

\begin{tabular}{|c|c|c|c|c|c|c|c|}
\hline Slot ATC & Voo & Origem & Destino & Eqpto & Tipo & Procedimento & Transição \\
\hline 7:06 & TAM3901 & SBRJ & & A319 & ARR & REDE 17 & LITRE \\
\hline $8: 15$ & GLO1311 & SBUL & & B737 & ARR & CELSO 17 & GRADE \\
\hline $8: 55$ & TAM3711 & SBBR & & А320 & ARR & CELSO 17 & GRADE \\
\hline $9: 17$ & TAM3909 & SBRJ & & A319 & ARR & REDE 17 & LITRE \\
\hline $9: 31$ & ONE6173 & & SBBR & F100 & DEP & DUMO & BGC \\
\hline $9: 57$ & GLO1280 & & SBFL & B737 & DEP & APIT & NIBGA \\
\hline $10: 21$ & TAM3720 & & SBBR & А320 & DEP & DUMO & $B G C$ \\
\hline $10: 24$ & VRN2153 & SBCT & & B737 & ARR & REDE 17 & PNG \\
\hline $10: 26$ & GLO1771 & SBGO & & B737 & ARR & CELSO 17 & GRADE \\
\hline $10: 31$ & GLO1384 & & SBGL & B738 & DEP & DIADEMA & PERES \\
\hline $11: 06$ & GLO1290 & & SBMG & B738 & DEP & KING & SCB \\
\hline $11: 36$ & GLO1519 & SBRJ & & B737 & ARR & REDE 17 & LITRE \\
\hline $11: 44$ & TAM3138 & & SBVT & A320 & DEP & DIADEMA & PERES \\
\hline $11: 46$ & VRN2100 & & SBPA & B738 & DEP & APIT & CURSE \\
\hline $12: 20$ & TAM3921 & SBRJ & & A319 & ARR & REDE 17 & LITRE \\
\hline $12: 36$ & PTN4733 & SBML & & AT42 & ARR & CELSO 17 & GRADE \\
\hline $13: 05$ & ONE6008 & & SBRJ & $\mathrm{F} 100$ & DEP & DIADEMA & COSME \\
\hline $13: 08$ & TAM3771 & SBCG & & А320 & ARR & CELSO 17 & GRADE \\
\hline $13: 29$ & TAM3061 & & SBPA & А320 & DEP & APIT & CURSE \\
\hline $14: 12$ & GLO1366 & & SBVT & B737 & DEP & DIADEMA & PERES \\
\hline $14: 25$ & TAM3271 & SBRP & & A320 & ARR & CELSO 17 & GRADE \\
\hline $14: 28$ & ТАМ3038 & SBJV & & A319 & ARR & REDE 17 & OSAMU \\
\hline $14: 47$ & TAM3010 & SBCT & & А320 & ARR & REDE 17 & PNG \\
\hline $14: 48$ & GLO1222 & & SBCT & B738 & DEP & APIT & NIBGA \\
\hline $14: 51$ & GLO1534 & & SBRJ & B737 & DEP & DIADEMA & COSME \\
\hline $16: 11$ & GLO1392 & & SBGL & B737 & DEP & DIADEMA & PERES \\
\hline $16: 18$ & GLO1344 & & SBCX & B737 & DEP & APIT & CURSE \\
\hline $16: 22$ & VRN2333 & SBSV & & B737 & ARR & CELSO 17 & PCL \\
\hline $16: 31$ & VRN2104 & & SBPA & B738 & DEP & APIT & CURSE \\
\hline $17: 10$ & PTN4769 & SJTC & & AT42 & ARR & CELSO 17 & GRADE \\
\hline $17: 16$ & GLO1380 & & SBJV & B737 & DEP & APIT & NIBGA \\
\hline $17: 32$ & GLO1285 & SBFL & & B737 & ARR & REDE 17 & OSAMU \\
\hline $17: 40$ & ONE6012 & & SBRJ & F100 & DEP & DIADEMA & COSME \\
\hline $18: 02$ & VRN2059 & SBCF & & B733 & ARR & CELSO 17 & PCL \\
\hline $18: 30$ & PTN4788 & & SBJF & AT42 & DEP & DIADEMA & PERES \\
\hline $18: 42$ & GLO1312 & & SBUL & B738 & DEP & DUMO & $B G C$ \\
\hline 19:05 & GLO1772 & & SBGO & B737 & DEP & DUMO & $B G C$ \\
\hline $19: 23$ & TAM3712 & & SBBR & A320 & DEP & DUMO & $B G C$ \\
\hline $19: 29$ & TAM3721 & SBBR & & A320 & ARR & CELSO 17 & GRADE \\
\hline $19: 57$ & TAM3727 & SBBR & & A319 & ARR & CELSO 17 & GRADE \\
\hline 20:01 & ONE6015 & SBRJ & & F100 & ARR & REDE 17 & LITRE \\
\hline 20:07 & TAM3956 & & SBRJ & A319 & DEP & DIADEMA & COSME \\
\hline
\end{tabular}




\begin{tabular}{cccccccc}
\hline Slot ATC & Voo & Origem & Destino & Eqpto & Tipo & Procedimento & Transição \\
\hline $20: 11$ & GLO1345 & SBCX & & B737 & ARR & REDE 17 & PNG \\
$20: 16$ & GLO1288 & & SBLO & B737 & DEP & KING & SCB \\
$21: 07$ & TAM3764 & SBLO & & A319 & ARR & CELSO 17 & GRADE \\
$21: 11$ & TAM3957 & SBRJ & & A319 & ARR & REDE 17 & LITRE \\
$21: 22$ & TAM3019 & & SBCT & A320 & DEP & APIT & NIBGA \\
$21: 24$ & PTN4789 & SBJF & & AT42 & ARR & REDE 17 & LITRE \\
$21: 42$ & GLO1266 & & SBCF & B737 & DEP & DUMO & TEXAS \\
$21: 58$ & TAM3736 & & SBSR & A319 & DEP & KING & SCB \\
\hline
\end{tabular}


C. 5 - Voos excluídos para redução de $15 \%$ no volume de tráfego

\begin{tabular}{|c|c|c|c|c|c|c|c|}
\hline Slot ATC & Voo & Origem & Destino & Eqpto & Tipo & Procedimento & Transição \\
\hline $6: 07$ & TAM3801 & SBCY & & А320 & ARR & CELSO 17 & GRADE \\
\hline $6: 19$ & GLO1500 & & SBRJ & B737 & DEP & DIADEMA & COSME \\
\hline 7:06 & TAM3901 & SBRJ & & A319 & ARR & REDE 17 & LITRE \\
\hline $7: 23$ & TAM3601 & SBSV & & A319 & ARR & CELSO 17 & PCL \\
\hline $7: 38$ & GLO1260 & & SBCF & B738 & DEP & DUMO & TEXAS \\
\hline $8: 12$ & TAM3048 & SBPA & & A320 & ARR & REDE 17 & OSAMU \\
\hline $8: 15$ & GLO1311 & SBUL & & B737 & ARR & CELSO 17 & GRADE \\
\hline $8: 17$ & PTN4738 & & SBML & AT42 & DEP & KING & SCB \\
\hline $8: 33$ & VRN2051 & SBCF & & B733 & ARR & CELSO 17 & PCL \\
\hline $8: 55$ & TAM3711 & SBBR & & А320 & ARR & CELSO 17 & GRADE \\
\hline $9: 10$ & TAM3201 & SBCF & & A319 & ARR & CELSO 17 & PCL \\
\hline $9: 17$ & TAM3909 & SBRJ & & A319 & ARR & REDE 17 & LITRE \\
\hline 9:30 & VRN2152 & & SBCT & B737 & DEP & APIT & NIBGA \\
\hline $9: 31$ & ONE6173 & & SBBR & $\mathrm{F} 100$ & DEP & DUMO & $B G C$ \\
\hline $9: 57$ & GLO1280 & & SBFL & B737 & DEP & APIT & NIBGA \\
\hline $10: 21$ & TAM3720 & & SBBR & А320 & DEP & DUMO & $B G C$ \\
\hline $10: 24$ & VRN2153 & SBCT & & B737 & ARR & REDE 17 & PNG \\
\hline $10: 26$ & GLO1771 & SBGO & & B737 & ARR & CELSO 17 & GRADE \\
\hline $10: 31$ & GLO1384 & & SBGL & B738 & DEP & DIADEMA & PERES \\
\hline $10: 56$ & GLO1377 & SBJV & & B737 & ARR & REDE 17 & OSAMU \\
\hline $11: 06$ & GLO1290 & & SBMG & B738 & DEP & KING & SCB \\
\hline $11: 11$ & TAM3920 & & SBRJ & A319 & DEP & DIADEMA & COSME \\
\hline $11: 13$ & TAM3917 & SBRJ & & A319 & ARR & REDE 17 & LITRE \\
\hline $11: 36$ & GLO1519 & SBRJ & & B737 & ARR & REDE 17 & LITRE \\
\hline $11: 44$ & TAM3138 & & SBVT & А320 & DEP & DIADEMA & PERES \\
\hline $11: 46$ & VRN2100 & & SBPA & B738 & DEP & APIT & CURSE \\
\hline $12: 08$ & GLO1282 & & SBFL & B737 & DEP & APIT & NIBGA \\
\hline $12: 20$ & TAM3921 & SBRJ & & A319 & ARR & REDE 17 & LITRE \\
\hline $12: 35$ & GLO1523 & SBRJ & & B737 & ARR & REDE 17 & LITRE \\
\hline $12: 36$ & PTN4733 & SBML & & AT42 & ARR & CELSO 17 & GRADE \\
\hline $13: 05$ & ONE6008 & & SBRJ & $\mathrm{F} 100$ & DEP & DIADEMA & cosme \\
\hline $13: 08$ & TAM3771 & SBCG & & А320 & ARR & CELSO 17 & GRADE \\
\hline $13: 29$ & TAM3061 & & SBPA & А320 & DEP & APIT & CURSE \\
\hline $13: 39$ & GLO1358 & & SBDN & B737 & DEP & KING & SCB \\
\hline $13: 46$ & GLO1243 & SBPA & & B737 & ARR & REDE 17 & OSAMU \\
\hline $14: 12$ & GLO1366 & & SBVT & B737 & DEP & DIADEMA & PERES \\
\hline $14: 22$ & GLO1223 & SBCT & & B737 & ARR & REDE 17 & PNG \\
\hline $14: 25$ & TAM3271 & SBRP & & А320 & ARR & CELSO 17 & GRADE \\
\hline $14: 28$ & TAM3038 & SBJV & & A319 & ARR & REDE 17 & OSAMU \\
\hline $14: 47$ & TAM3010 & SBCT & & A320 & ARR & REDE 17 & PNG \\
\hline $14: 48$ & GLO1222 & & SBCT & B738 & DEP & APIT & NIBGA \\
\hline $14: 51$ & GLO1534 & & SBRJ & B737 & DEP & DIADEMA & COSME \\
\hline
\end{tabular}




\begin{tabular}{|c|c|c|c|c|c|c|c|}
\hline Slot ATC & Voo & Origem & Destino & Eqpto & Tipo & Procedimento & Transição \\
\hline 15:06 & TAM3203 & SBCF & & A320 & ARR & CELSO 17 & PCL \\
\hline $16: 11$ & GLO1392 & & SBGL & B737 & DEP & DIADEMA & PERES \\
\hline $16: 18$ & GLO1344 & & SBCX & B737 & DEP & APIT & CURSE \\
\hline $16: 22$ & VRN2333 & SBSV & & B737 & ARR & CELSO 17 & PCL \\
\hline $16: 31$ & VRN2104 & & SBPA & B738 & DEP & APIT & CURSE \\
\hline $16: 35$ & TAM3939 & SBRJ & & A319 & ARR & REDE 17 & LITRE \\
\hline $17: 10$ & PTN4769 & SJTC & & AT42 & ARR & CELSO 17 & GRADE \\
\hline $17: 16$ & GLO1380 & & SBJV & B737 & DEP & APIT & NIBGA \\
\hline $17: 24$ & GLO1369 & SBVT & & B737 & ARR & REDE 17 & LITRE \\
\hline $17: 32$ & GLO1285 & SBFL & & B737 & ARR & REDE 17 & OSAMU \\
\hline $17: 40$ & ONE6012 & & SBRJ & F100 & DEP & DIADEMA & cosme \\
\hline $18: 02$ & VRN2059 & SBCF & & B733 & ARR & CELSO 17 & PCL \\
\hline $18: 30$ & PTN4788 & & SBJF & AT42 & DEP & DIADEMA & PERES \\
\hline $18: 33$ & GLO1226 & & SBCT & B738 & DEP & APIT & NIBGA \\
\hline $18: 42$ & GLO1312 & & SBUL & B738 & DEP & DUMO & $B G C$ \\
\hline 19:01 & VRN2106 & & SBPA & B733 & DEP & APIT & CURSE \\
\hline 19:05 & GLO1772 & & SBGO & B737 & DEP & DUMO & $B G C$ \\
\hline $19: 23$ & TAM3712 & & SBBR & А320 & DEP & DUMO & $B G C$ \\
\hline 19:25 & GLO1381 & SBJV & & B737 & ARR & REDE 17 & OSAMU \\
\hline $19: 29$ & TAM3721 & SBBR & & А320 & ARR & CELSO 17 & GRADE \\
\hline $19: 57$ & TAM3727 & SBBR & & A319 & ARR & CELSO 17 & GRADE \\
\hline 20:01 & ONE6015 & SBRJ & & F100 & ARR & REDE 17 & LITRE \\
\hline $20: 07$ & TAM3956 & & SBRJ & A319 & DEP & DIADEMA & COSME \\
\hline $20: 11$ & GLO1345 & SBCX & & B737 & ARR & REDE 17 & PNG \\
\hline $20: 16$ & GLO1288 & & SBLO & B737 & DEP & KING & SCB \\
\hline $20: 24$ & GLO1213 & SBBR & & B738 & ARR & CELSO 17 & GRADE \\
\hline $21: 03$ & TAM3800 & & SBCY & А320 & DEP & KING & SCB \\
\hline $21: 07$ & TAM3764 & SBLO & & A319 & ARR & CELSO 17 & GRADE \\
\hline $21: 11$ & TAM3957 & SBRJ & & A319 & ARR & REDE 17 & LITRE \\
\hline $21: 22$ & TAM3019 & & SBCT & АЗ20 & DEP & APIT & NIBGA \\
\hline $21: 24$ & PTN4789 & SBJF & & AT42 & ARR & REDE 17 & LITRE \\
\hline 21:42 & GLO1266 & & SBCF & B737 & DEP & DUMO & TEXAS \\
\hline $21: 58$ & TAM3736 & & SBSR & A319 & DEP & KING & SCB \\
\hline
\end{tabular}


C.6 - Voos com horários reorganizados

\begin{tabular}{|c|c|c|c|c|c|c|c|}
\hline Slot ATC & Voo & Origem & Destino & Eqpto & Tipo & Procedimento & Transição \\
\hline $6: 15$ & TAM3900 & & SBRJ & A319 & DEP & DIADEMA & COSME \\
\hline $6: 17$ & GLO1375 & SBDN & & B737 & ARR & CELSO 17 & GRADE \\
\hline $6: 21$ & TAM3605 & SBSV & & A320 & ARR & CELSO 17 & PCL \\
\hline $6: 23$ & GLO1346 & & SBVT & B737 & DEP & DIADEMA & PERES \\
\hline $7: 56$ & TAM3716 & & SBBR & А320 & DEP & DUMO & $B G C$ \\
\hline $7: 58$ & PTN4749 & SJTC & & AT42 & ARR & CELSO 17 & GRADE \\
\hline $8: 25$ & TAM3460 & & SBGO & А320 & DEP & DUMO & $B G C$ \\
\hline $8: 27$ & TAM3101 & & SBFL & A319 & DEP & APIT & NIBGA \\
\hline $8: 44$ & ONE6173 & SBFL & & $\mathrm{F} 100$ & ARR & REDE 17 & OSAMU \\
\hline $8: 46$ & GLO1242 & & SBPA & B737 & DEP & APIT & CURSE \\
\hline $8: 48$ & PTN4782 & & SBJF & AT42 & DEP & DIADEMA & PERES \\
\hline $10: 04$ & TAM3660 & & SBIL & A319 & DEP & DUMO & TEXAS \\
\hline $10: 06$ & GLO1513 & SBRJ & & B737 & ARR & REDE 17 & LITRE \\
\hline $10: 23$ & VRN2153 & SBCT & & B737 & ARR & REDE 17 & PNG \\
\hline $10: 24$ & PTN4712 & & SBML & AT42 & DEP & KING & SCB \\
\hline $10: 29$ & TAM3254 & & SBGL & А320 & DEP & DIADEMA & PERES \\
\hline $10: 30$ & PTN4772 & SJTC & & AT42 & ARR & CELSO 17 & GRADE \\
\hline $10: 36$ & GLO1515 & SBRJ & & B737 & ARR & REDE 17 & LITRE \\
\hline $10: 38$ & GLO1378 & & SBJV & B737 & DEP & APIT & NIBGA \\
\hline $10: 44$ & TAM3918 & & SBRJ & A319 & DEP & DIADEMA & cosme \\
\hline $10: 43$ & TAM3007 & & SBCT & A319 & DEP & APIT & NIBGA \\
\hline $11: 38$ & GLO1310 & & SBUL & B737 & DEP & DUMO & $B G C$ \\
\hline $11: 40$ & TAM3922 & & SBRJ & A319 & DEP & DIADEMA & cosme \\
\hline $11: 42$ & VRN2053 & SBCF & & B733 & ARR & CELSO 17 & PCL \\
\hline $12: 04$ & GLO1220 & & SBCT & B737 & DEP & APIT & NIBGA \\
\hline $12: 06$ & GLO1521 & SBRJ & & B737 & ARR & REDE 17 & LITRE \\
\hline 13:05 & TAM3925 & SBRJ & & A319 & ARR & REDE 17 & LITRE \\
\hline $13: 06$ & ONE6008 & & SBRJ & $\mathrm{F} 100$ & DEP & DIADEMA & cosme \\
\hline $13: 54$ & GLO1371 & SBCY & & B738 & ARR & CELSO 17 & GRADE \\
\hline $13: 56$ & GLO1262 & & SBCF & B738 & DEP & DUMO & TEXAS \\
\hline $14: 00$ & GLO1529 & SBRJ & & B737 & ARR & REDE 17 & LITRE \\
\hline $14: 02$ & GLO1396 & & SBSV & B737 & DEP & DUMO & TEXAS \\
\hline $14: 18$ & VRN2055 & SBCF & & B733 & ARR & CELSO 17 & PCL \\
\hline $14: 20$ & GLO1532 & & SBRJ & B737 & DEP & DIADEMA & COSME \\
\hline $15: 08$ & TAM3936 & & SBRJ & A319 & DEP & DIADEMA & COSME \\
\hline $15: 12$ & VRN2054 & & SBCF & B733 & DEP & DUMO & TEXAS \\
\hline $15: 15$ & TAM3933 & SBRJ & & A319 & ARR & REDE 17 & LITRE \\
\hline $15: 22$ & GLO1353 & SBGO & & B737 & ARR & CELSO 17 & GRADE \\
\hline $15: 24$ & TAM3222 & & SBCF & A320 & DEP & DUMO & TEXAS \\
\hline $15: 35$ & GLO1538 & & SBRJ & B737 & DEP & DIADEMA & COSME \\
\hline $15: 37$ & TAM3055 & & SBPA & АЗ20 & DEP & APIT & CURSE \\
\hline $15: 54$ & TAM3202 & & SBCF & А320 & DEP & DUMO & TEXAS \\
\hline
\end{tabular}




\begin{tabular}{|c|c|c|c|c|c|c|c|}
\hline Slot ATC & Voo & Origem & Destino & Eqpto & Tipo & Procedimento & Transição \\
\hline $15: 56$ & VRN2107 & SBPA & & B738 & ARR & REDE 17 & OSAMU \\
\hline $16: 13$ & TAM3060 & SBPA & & A320 & ARR & REDE 17 & OSAMU \\
\hline $16: 14$ & GLO1540 & & SBRJ & B737 & DEP & DIADEMA & COSME \\
\hline $16: 20$ & VRN2333 & SBSV & & B737 & ARR & CELSO 17 & PCL \\
\hline $16: 22$ & VRN2132 & & SBFL & B737 & DEP & APIT & NIBGA \\
\hline $17: 14$ & GLO1380 & & SBJV & B737 & DEP & APIT & NIBGA \\
\hline $17: 16$ & TAM3134 & & SBVT & A320 & DEP & DIADEMA & PERES \\
\hline $17: 18$ & TAM3661 & SBIL & & A319 & ARR & CELSO 17 & PCL \\
\hline $17: 22$ & GLO1369 & SBVT & & B737 & ARR & REDE 17 & LITRE \\
\hline $17: 24$ & GLO1544 & & SBRJ & B737 & DEP & DIADEMA & COSME \\
\hline $17: 35$ & TAM3946 & & SBRJ & A319 & DEP & DIADEMA & COSME \\
\hline $17: 37$ & TAM3943 & SBRJ & & A319 & ARR & REDE 17 & LITRE \\
\hline $18: 40$ & GLO1312 & & SBUL & B738 & DEP & DUMO & $B G C$ \\
\hline $18: 42$ & GLO1355 & SBGO & & B737 & ARR & CELSO 17 & GRADE \\
\hline $19: 12$ & VRN2061 & SBCF & & B738 & ARR & CELSO 17 & PCL \\
\hline $19: 15$ & GLO1552 & & SBRJ & B737 & DEP & DIADEMA & COSME \\
\hline $19: 19$ & TAM3238 & & SBUL & A319 & DEP & DUMO & $B G C$ \\
\hline $19: 21$ & GLO1381 & SBJV & & B737 & ARR & REDE 17 & OSAMU \\
\hline $19: 23$ & GLO1210 & & SBBR & B738 & DEP & DUMO & $B G C$ \\
\hline $19: 25$ & GLO1551 & SBRJ & & B737 & ARR & REDE 17 & LITRE \\
\hline $19: 27$ & TAM3712 & & SBBR & A320 & DEP & DUMO & $B G C$ \\
\hline $19: 36$ & TAM3954 & & SBRJ & A319 & DEP & DIADEMA & COSME \\
\hline $19: 38$ & TAM3951 & SBRJ & & A319 & ARR & REDE 17 & LITRE \\
\hline $20: 45$ & VRN2133 & SBFL & & B737 & ARR & REDE 17 & OSAMU \\
\hline $20: 48$ & ONE6016 & & SBRJ & F100 & DEP & DIADEMA & COSME \\
\hline $20: 50$ & ONE6172 & SBBR & & F100 & ARR & CELSO 17 & GRADE \\
\hline $20: 52$ & TAM3105 & & SBFL & A319 & DEP & APIT & NIBGA \\
\hline $20: 54$ & GLO1557 & SBRJ & & B737 & ARR & REDE 17 & LITRE \\
\hline $20: 55$ & GLO1558 & & SBRJ & B737 & DEP & DIADEMA & COSME \\
\hline $20: 57$ & VRN2108 & & SBPA & B738 & DEP & APIT & CURSE \\
\hline $21: 55$ & PTN4760 & & SBAU & AT42 & DEP & KING & SCB \\
\hline $21: 57$ & GLO1273 & SBCF & & B738 & ARR & CELSO 17 & PCL \\
\hline $22: 07$ & TAM3774 & & SBCG & A319 & DEP & KING & SCB \\
\hline 22:09 & TAM3960 & & SBRJ & A319 & DEP & DIADEMA & COSME \\
\hline
\end{tabular}

